\title{
Measurable chromatic and independence numbers for ergodic graphs and group actions
}

\author{
Clinton T. Conley and Alexander S. Kechris
}

\begin{abstract}
We study in this paper combinatorial problems concerning graphs generated by measure preserving actions of countable groups on standard measure spaces. In particular we study chromatic and independence numbers, in both the measure-theoretic and the Borel context, and relate the behavior of these parameters to properties of the acting group such as amenability, Kazhdan's property (T), and freeness. We also prove a Borel analog of the classical Brooks' Theorem in finite combinatorics for actions of groups with finitely many ends.
\end{abstract}

Mathematics Subject Classification (2010). 03E15, 22F10, 05C15, 37A15, 37A25.

Keywords. Ergodic actions, Borel combinatorics, chromatic numbers, amenable groups, free groups.

\section{Contents}

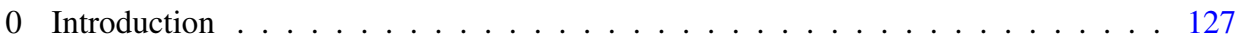

1 Preliminaries . . . . . . . . . . . . . . . . . . . . . 133

2 Chromatic and independence numbers . . . . . . . . . . . . . . . . 135

3 Hyperfinite graphs . . . . . . . . . . . . . . . . . . . . . . . . . . . . . 144

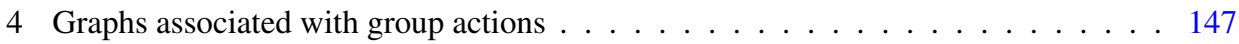

5 On Brooks' Theorem . . . . . . . . . . . . . . . . . . . . . . . . . 167

6 A matching problem . . . . . . . . . . . . . . . . . . . . . 174

References . . . . . . . . . . . . . . . . . . . . . 178

\section{Introduction}

We study in this paper some combinatorial invariants associated with ergodic actions of infinite, countable (discrete) groups.

Let $(X, \mu)$ be a standard probability space and $\Gamma$ an infinite, countable group with a set of generators $1 \notin S \subseteq \Gamma$. Given a free, measure-preserving action $a$ of $\Gamma$ on $(X, \mu)$, we consider the graph $G(S, a)=(X, E(S, a))$, whose vertices are the points 
in $X$ and where $x \neq y \in X$ are adjacent if there is a generator $s \in S$ taking one to the other. It is clear that the connected components of this graph are isomorphic to the Cayley graph $\operatorname{Cay}(\Gamma, S)$ and thus parameters such as the chromatic number of $G(S, a)$ are identical to those of $\operatorname{Cay}(\Gamma, S)$. This however requires selecting an element from each connected component and thus essentially depends upon a use of the Axiom of Choice. However, the situation is vastly different when one considers instead measurable colorings and the associated measurable chromatic numbers.

Let us introduce first the combinatorial invariants we will be interested in.

Consider a locally countable Borel graph $G=(X, E)$ on a standard probability space $(X, \mu)$. We denote by $E^{*}$ the associated Borel equivalence relation whose classes are the connected components of $G$. Given a property of equivalence relations $\mathcal{P}$, we say that $G$ has property $\mathcal{P}$ if $E^{*}$ has property $\mathcal{P}$. This explains what it means to say that $G$ is $(\mu$-)measure preserving, ergodic, hyperfinite, smooth, etc. In particular, the graphs $G(S, a)$ discussed before are measure preserving, and they are ergodic iff the action $a$ is ergodic.

Given such a graph $G=(X, E)$ its $\left(\mu\right.$-)measurable chromatic number, $\chi_{\mu}(G)$, is the smallest cardinality of a standard Borel space $Y$ for which there is a $(\mu$-)measurable coloring $c: X \rightarrow Y$ (i.e., $x E y \Rightarrow c(x) \neq c(y)$ ). Clearly $\chi_{\mu}(G) \in$ $\left\{1,2,3, \ldots, \aleph_{0}, 2^{\aleph_{0}}\right\}$. It is well known (see, e.g., [26]) that there are acyclic such graphs $G$, for which of course the usual chromatic number $\chi(G)$ is equal to 2, with $\chi_{\mu}(G)=2^{\aleph_{0}}$.

In addition, we consider the approximate ( $\mu$-)measurable chromatic number, $\chi_{\mu}^{\mathrm{ap}}(G)$, which is defined as the smallest cardinality of a standard Borel space $Y$ such that for each $\varepsilon>0$, there is a Borel set $A \subseteq X$ with $\mu(X \backslash A)<\varepsilon$ and a measurable coloring $c: A \rightarrow Y$ of the induced subgraph $G \mid A$. Clearly $\chi_{\mu}^{\text {ap }}(G) \leq \chi_{\mu}(G)$.

Finally, the ( $\mu$-)independence number of $G, i_{\mu}(G)$, is the supremum of the measures of independent Borel sets $(A \subseteq X$ is independent if no two elements of $A$ are adjacent in $G)$. Clearly $i_{\mu}(G) \in[0,1]$. It is easy to check that $\chi_{\mu}^{\mathrm{ap}}(G) \geq \frac{1}{i_{\mu}(G)}$, so graphs with small independence number have large (approximate) measurable chromatic number.

We discuss in $\S 2$ various examples of measure-preserving, ergodic graphs $G$ with small chromatic number $\chi(G)$ (e.g., acyclic) but for which $\chi_{\mu}^{\text {ap }}(G)$ or $\chi_{\mu}(G)$ take various finite or infinite values, and others in which $i_{\mu}(G)$ takes any value in $[0,1)$ (the value 1 can be easily seen to be impossible to realize in such a graph).

However for graphs of bounded degree, there are further restrictions (see 2.19, 2.20), which are analogs of the classical Brooks' Theorem in finite graph theory (see, e.g., Diestel [10]), which asserts that for finite graphs $G$ the chromatic number is bounded by the maximum degree $d$ of the graph, unless $d=2$ and $G$ contains an odd cycle or $d \geq 3$ and $G$ contains a complete graph of size $d+1$.

Theorem 0.1. Let $(X, \mu)$ be a standard probability space and $G=(X, E)$ a Borel graph with degree bounded by $d \geq 2$. Then 
(i) $\chi_{\mu}(G) \leq d+1$ and thus $i_{\mu}(G) \geq 1 /(d+1)([26])$;

(ii) if $d=2$ and $G$ has no odd cycles (i.e., $G$ is bipartite) or else $d \geq 3$ and $G$ has no cliques (i.e., complete subgraphs) of size $d+1$, then $\chi_{\mu}^{\mathrm{ap}}(G) \leq d$ and thus $i_{\mu}(G) \geq 1 / d$.

In $\$ 3$, we consider the case of hyperfinite graphs. Denote below by $\chi^{*}(G)$ the smallest chromatic number of an induced subgraph $G \mid A$, where $A$ is an $E^{*}$-invariant Borel set of measure 1. In particular, $\chi^{*}(G) \leq \chi(G)$. Using some techniques of Miller [39], we show (see 3.1, 3.8):

Theorem 0.2. Let $(X, \mu)$ be a standard probability space and $G$ a locally countable, acyclic, $\left(\mu\right.$-)hyperfinite graph. Then $\chi_{\mu}^{\mathrm{ap}}(G) \leq 2$ and thus $i_{\mu}(G) \geq 1 / 2$. If moreover $G$ is locally finite, $(\mu-)$ hyperfinite, but not necessarily acyclic, then $\chi_{\mu}^{\mathrm{ap}}(G) \leq \chi^{*}(G)$ and thus $i_{\mu}(G) \geq 1 / \chi^{*}(G)$, and if $G$ is also measure preserving, then $\chi_{\mu}^{\mathrm{ap}}(G)=$ $\chi^{*}(G)$.

In $\$ 4$ we consider the graphs associated with group actions, as discussed in the beginning of this introduction. Let $\chi_{\mu}(S, a), \chi_{\mu}^{\mathrm{ap}}(S, a), i_{\mu}(S, a)$ be the parameters associated with $G(S, a)$. It is easy to see that $i_{\mu}(S, a) \leq 1 / 2$.

Let $a \prec b$ be the relation of weak containment among measure preserving actions of $\Gamma$ on $(X, \mu)$; see [23]. We have $a \prec b$ iff $a$ is in the closure, in the weak topology, of the conjugacy class of $b$. We now have the following monotonicity properties (see 4.2, 4.3).

Theorem 0.3. Let $\Gamma$ be a countable group and $S$ a finite set of generators. Then

$$
a \prec b \Longrightarrow i_{\mu}(S, a) \leq i_{\mu}(S, b), \chi_{\mu}^{\mathrm{ap}}(S, a) \geq \chi_{\mu}^{\mathrm{ap}}(S, b) .
$$

It follows that $i_{\mu}(S, a), \chi_{\mu}^{\mathrm{ap}}(S, a)$ are invariants of weak equivalence, $a \sim b$, where $a \sim b \Longleftrightarrow a \prec b$ and $b \prec a$.

Note that Cay $(\Gamma, S)$ is bipartite iff there is no odd length word in $S \cup S^{-1}$ equal to the identity in $\Gamma$. We show in 4.5 that (for any $\Gamma, S)$ if $\operatorname{Cay}(\Gamma, S)$ is not bipartite, then $i_{\mu}(S, a)<1 / 2$ and $\chi_{\mu}^{\mathrm{ap}}(S, a) \geq 3$. In fact in this case $i_{\mu}(S, a) \leq 1 / 2-1 /(2 g)$, where $g$ is the odd girth (= length of shortest odd cycle) in $\operatorname{Cay}(\Gamma, S)$. From this and 0.1 it follows that for $\Gamma=(\mathbb{Z} / 2 \mathbb{Z}) *(\mathbb{Z} / 3 \mathbb{Z})=\left\langle s, t \mid s^{2}=1, t^{3}=1\right\rangle$ and $S=\{s, t\}$, we have $i_{\mu}(S, a)=1 / 3$ for every free, measure-preserving action $a$ of $\Gamma$.

It is known, see, e.g., [23], 13.2, that any two free, measure-preserving, ergodic actions of an amenable group $\Gamma$ are weakly equivalent, thus for any finite generating set $S \subseteq \Gamma$, we have that

$$
i_{\mu}(\Gamma, S)=i_{\mu}(S, a), \quad \chi_{\mu}^{\mathrm{ap}}(\Gamma, S)=\chi_{\mu}^{\mathrm{ap}}(S, a)
$$

are independent of the action $a$. We can identify $i_{\mu}(\Gamma, S), \chi_{\mu}^{\mathrm{ap}}(\Gamma, S)$ in terms of $\operatorname{Cay}(\Gamma, S)$. For a finite graph $G=(X, E)$, we define the independence ratio $i(G)$ 
to be $i_{\mu}(G)$, where $\mu$ is the normalized counting measure on $X$ (so $i(G)=\frac{\alpha(G)}{|X|}$, where $\alpha(G)$ is the maximum cardinality of an independent subset of $X)$. For $(\Gamma, S)$ as above and finite $F \subseteq \Gamma$, let $i(F, S)$ be the independence ratio of the induced subgraph $\operatorname{Cay}(\Gamma, S) \mid F$. Let $\left(F_{n}\right)$ be a Følner sequence for $\Gamma$, i.e., $F_{n} \subseteq \Gamma$ are finite, non-empty, and $\frac{\left|\gamma F_{n} \Delta F_{n}\right|}{\left|F_{n}\right|} \rightarrow 0$ for all $\gamma \in \Gamma$. Using a result that can be proved easily using the quasi-tiling machinery of Ornstein-Weiss [43] (see Gromov [14], 1.3, and Lindenstrauss-Weiss [31], Appendix), one can show that for any Følner sequence $\left(F_{n}\right)$,

$$
\lim _{n \rightarrow \infty} i\left(F_{n}, S\right)=i(\Gamma, S)
$$

exists (and is of course independent of $\left(F_{n}\right)$ ). We call this the independence number of $\operatorname{Cay}(\Gamma, S)$. We now have (see $4.7,4.10$ )

Theorem 0.4. Let $\Gamma$ be a countable, amenable group and $S$ a finite set of generators. Then:

(i) $\chi_{\mu}^{\mathrm{ap}}(\Gamma, S)=\chi(\operatorname{Cay}(\Gamma, S))$,

(ii) $i_{\mu}(\Gamma, S)=i(\Gamma, S)$.

For non-amenable $\Gamma, i_{\mu}(S, a)$ and $\chi_{\mu}^{\mathrm{ap}}(S, a)$ are not necessarily constant. In fact for any $\Gamma$ and finite set of generators $S$ with $\operatorname{Cay}(\Gamma, S)$ bipartite, we have the following characterization of amenability (see 4.13, 4.14).

Theorem 0.5. Let $\Gamma$ be a countable group and $S \subseteq \Gamma$ a finite set of generators with Cay $(\Gamma, S)$ bipartite. Then the following are equivalent:

(i) $\Gamma$ is amenable.

(ii) $i_{\mu}(S, a)$ is constant for any free, measure-preserving action a of $\Gamma$.

(iii) $i_{\mu}(S, a)=1 / 2$, for any free, measure-preserving action a of $\Gamma$.

(iv) $\chi_{\mu}^{\text {ap }}(S, a)$ is constant for any free, measure-preserving action a of $\Gamma$.

(v) $\chi_{\mu}^{\mathrm{ap}}(S, a)=2$, for every free, measure-preserving action a of $\Gamma$.

Subsequently an alternative proof of this result was given in Abért-Elek [1].

We also have an analogous characterization of groups that have property $(\mathrm{T})$ and the Haagerup Approximation Property HAP (see 4.15).

Theorem 0.6. Let $\Gamma$ be an infinite, countable group and $S \subseteq \Gamma$ a finite set of generators such that $\operatorname{Cay}(\Gamma, S)$ is bipartite. Then the following are equivalent:

(i) $\Gamma$ has property $(\mathrm{T})$.

(ii) $i_{\mu}(S, a)<1 / 2$, for every free, measure-preserving, weakly mixing action a of $\Gamma$.

(iii) $\chi_{\mu}^{\mathrm{ap}}(S, a) \geq 3$, for every free, measure-preserving, weakly mixing action a of $\Gamma$. 
Also the following are equivalent:

(i*) $\Gamma$ does not have the HAP.

(ii*) $i_{\mu}(S, a)<1 / 2$, for every free, measure-preserving, mixing action a of $\Gamma$.

(iii*) $\chi_{\mu}^{\mathrm{ap}}(S, a) \geq 3$, for every free, measure-preserving, mixing action a of $\Gamma$.

We next consider the shift action $s_{\Gamma}$ of the free group $\Gamma=\mathbb{F}_{m}$, with $m$ free generators $S=\left\{a_{1}, \ldots, a_{m}\right\}$, on $2^{\Gamma}$ with the product measure. Using a result of Kesten [28] for the norm of averaging operators, we show the following result (see 4.17).

Theorem 0.7. Let $\Gamma=\mathbb{F}_{m}$ be the free group with a free set of generators $S$ and let $s_{\Gamma}$ be its shift action on $2^{\Gamma}$. Then

$$
\frac{1}{2 m} \leq i_{\mu}\left(S, s_{\Gamma}\right) \leq \frac{\sqrt{2 m-1}}{m+\sqrt{2 m-1}},
$$

and

$$
2 m \geq \chi_{\mu}^{\mathrm{ap}}\left(S, s_{\Gamma}\right) \geq \frac{m+\sqrt{2 m-1}}{\sqrt{2 m-1}} .
$$

This has the following consequence (see 2.5), answering a question in [39].

Corollary 0.8. For each $2 \leq n<\aleph_{0}$, there is an acyclic, bounded degree, measurepreserving, ergodic Borel graph $G$ with $\chi_{\mu}(G)=n$.

Without the requirements of having bounded degree or preserving measure, such examples were first found by Laczkovich (see [26], Appendix).

The exact values of $i_{\mu}\left(S, s_{\Gamma}\right), \chi_{\mu}^{\mathrm{ap}}\left(S, s_{\Gamma}\right)$ in 0.7 are unknown. It should be noted that there is no known example of $(\Gamma, S)$, with $\Gamma$ amenable and $S$ finite, for which $\chi_{\mu}\left(S, s_{\Gamma}\right)>\chi(\operatorname{Cay}(\Gamma, S))+1$. For instance, Gao-Jackson-Miller (unpublished) and recently Adam Timar (private communication) have shown that for $\Gamma=\mathbb{Z}^{m}$, with $S$ the usual set of generators (for which of course $\chi(\operatorname{Cay}(\Gamma, S))=2), \chi_{\mu}\left(S, s_{\Gamma}\right)=3$.

We also present in $\S 4$ other examples of free, measure-preserving, ergodic actions of $\mathbb{F}_{m}$ that satisfy the bounds in 0.7 .

Finally in $\S 4$ we discuss, for any $(\Gamma, S)$, canonical finite graphs that "approximate" the infinite graph $G\left(S, s_{\Gamma}\right)$ associated with the shift of $\Gamma$ on $2^{\Gamma}$. Labeled and "weighted" versions of these graphs were also used in Bowen [6]. Applying this to $\Gamma=\mathbb{F}_{m}$ and a free set of generators $S$ produces a natural explicit family of finite graphs $G_{n, m, k}(n, m, k \geq 1)$ which simultaneously have arbitrarily large odd girth $g_{\text {odd }}(G)$ and arbitrarily small independence ratio $i(G)$, thus arbitrarily large chromatic numbers. Such explicit families appear to be of interest in finite graph theory. More precisely, we have (see 4.22): 
Theorem 0.9. There is an explicit family of finite graphs $G_{m, k, n}(m, k, n \geq 1)$ and a map $m, k \mapsto N(m, k)$ such that for any $m, k$, if $n>N(m, k)$, then

$$
\begin{aligned}
g_{\text {odd }}\left(G_{m, k, n}\right) & >k, \\
i\left(G_{m, k, n}\right) & \leq \frac{2 \sqrt{2 m-1}}{m+\sqrt{2 m-1}},
\end{aligned}
$$

and thus

$$
\chi\left(G_{m, k, n}\right) \geq \frac{m+\sqrt{2 m-1}}{2 \sqrt{2 m-1}} .
$$

In $\$ 5$ we return to Brooks' Theorem and study Borel analogs of it, especially in the context of graphs generated by group actions.

Let $\Gamma$ be an infinite countable group and $1 \notin S$ a finite set of generators for $\Gamma$. Let $d=\left|S^{ \pm 1}\right|$, where $S^{ \pm 1}=S \cup S^{-1}$, be the degree of the Cayley graph of $\Gamma, S$. Let $A$ be a free Borel action of $\Gamma$ on a standard Borel space $X$ (we do not assume here that a measure is necessarily present on $X)$. We can define as before the graph $G(S, A)$ associated with this action and the set of generators $S$. We denote be $\chi_{B}(S, A)$ the Borel chromatic number of this graph, i.e., the smallest cardinality of a standard Borel space $Y$ for which there is a Borel coloring $c: X \rightarrow Y$ for $G(S, A)$. It follows from results of [26] that $\chi_{B}(S, A) \leq d+1$. We examine under what circumstances this can be improved to $\chi_{B}(S, A) \leq d$ as in Brooks' Theorem. We show the following (see 5.12)

Theorem 0.10. Suppose that $\Gamma$ is a finitely generated infinite group isomorphic neither to $\mathbb{Z}$ nor to $(\mathbb{Z} / 2 \mathbb{Z}) *(\mathbb{Z} / 2 \mathbb{Z})$. Suppose further that $\Gamma$ has finitely many ends. Let $S$ be a finite set of generators for $\Gamma$ and put $d=\left|S^{ \pm 1}\right|$. Then for any free Borel action $A$ of $\Gamma$ on a standard Borel space $X$, we have $\chi_{B}(S, A) \leq d$.

The requirement that $\Gamma$ is not isomorphic to $\mathbb{Z}$ or to $(\mathbb{Z} / 2 \mathbb{Z}) *(\mathbb{Z} / 2 \mathbb{Z})$ is necessary as the free part of the shift action of these groups $\Gamma$ on $2^{\Gamma}$ has Borel chromatic number equal to 3 (with respect to the usual set of generators). Groups that satisfy the hypotheses of the preceding theorem include: groups with property $(\mathrm{T})$, direct products of two infinite groups, amenable groups, and, more generally, groups of cost 1 , etc. In particular, if $\Gamma$ is a 2 -generated such group, then for any free Borel action of $\Gamma$ that admits an invariant Borel probability measure with respect to which it is weakly mixing, the corresponding Borel chromatic number is either 3 or 4 . This extends a result of Gao-Jackson [12] and Miller, who proved this for the free part of the shift action of $\mathbb{Z}^{2}$ on $2^{\mathbb{Z}^{2}}$ (see $\S 4$ ).

In the last section $\S 6$ we discuss a matching problem in the Borel and measurable contexts related to earlier work of Laczkovich [30] and Kłopotowski-NadkarniSarbadhikari-Srivastava [29]. 
Addendum. After the first version of this paper has been completed, we received a preliminary draft of a paper by Lyons and Nazarov [35], with subject matter closely related to this paper. In particular, it contains a version of Proposition 4.16 below. Also, its main result, which is that the graph associated with the shift action of a non-amenable group $\Gamma$, and a finite set of generators $S \subseteq \Gamma$, on $[0,1]^{\Gamma}$ admits a measurable matching, provides the solution to a problem that we discussed in $\S 6$ of the original version. Moreover, Lyons (private communication) mentioned that they have also considered the finite graphs approximating the graphs $G\left(S, s_{\Gamma}\right)$, discussed in $\S 4$, although these do not appear in [35]. Finally in [35] the authors mention that earlier results of Frieze-Luczak on random graphs imply that for large values of $m$, $i_{\mu}\left(S, s_{\Gamma}\right) \leq \frac{\log 2 m}{m}$ and thus $\chi_{\mu}^{\mathrm{ap}}\left(S, s_{\Gamma}\right) \geq \frac{m}{\log 2 m}$.

Acknowledgments. The authors would like to thank M. Abért, G. Elek, G. Hjorth, A. Ioana, R. Lyons, B. Miller, Y. Shalom, B. Sudakov, S. Thomas, B. Weiss and the referees for many useful conversations and suggestions, and wish to extend additional thanks to B. Miller for allowing inclusion of Lemma 3.2. M. Abért and G. Elek pointed out an error in our original proof of a version of Theorem 0.1 (ii). This has now been repaired by an alternative argument (see 2.19, 2.20). G. Elek has also independently suggested a somewhat related proof. A. S. Kechris was partially supported by NSF Grant DMS-0968710, the E. Schrödinger Institute, Vienna, and the Mittag-Leffler Institute, Djursholm.

\section{Preliminaries}

(A) A graph is a pair $G=(X, E)$, where $X$ is a set whose elements we call vertices of $G$ and $E \subseteq X^{2}$ satisfies: $(x, x) \notin E$ (i.e., there are no loops) and $(x, y) \in E \Longleftrightarrow$ $(y, x) \in E$ (i.e., the graph is symmetric). We often write $x E y$ to denote $(x, y) \in E$, and we identify $E$ with the set of unordered pairs $\{\{x, y\}: x E y\}$, which we call the edges of $G$. If $x E y$ we say that $x, y$ are adjacent.

Occasionally we will also consider graphs with (possible) loops, those in which $(x, x) \in E$ is allowed, for some $x \in X$, and directed graphs, those in which $E \subseteq X^{2}$ is not necessarily symmetric.

A path in $G$ is a sequence $x_{0}, x_{1}, \ldots, x_{n}, n \geq 1$, of distinct vertices such that $x_{i} E x_{i+1}, 0 \leq i<n$. The length of such a path is the number of edges it uses, so the length of $x_{0}, x_{1}, \ldots, x_{n}$ is $n$. Such a path is a cycle if $n \geq 2$ and, moreover, $x_{n} E x_{0}$; its length is $n+1$.

We denote by $E^{*}$ the smallest equivalence relation containing $E$. Its equivalence classes are the connected components of $G$, and thus two vertices $x, y$ are connected exactly when $x=y$ or there is a path $x=x_{0}, x_{1}, \ldots, x_{n}=y$. If $x, y$ are connected, we set $\rho_{G}(x, y)$ equal to the length of the shortest path from $x$ to $y$, and call it the $(G)$-distance from $x$ to $y$. The graph is connected if it has a unique connected component. 
A graph is acyclic if it contains no cycles; we sometimes call such graphs forests and their connected components trees. One easily sees that if $x, y$ are distinct vertices of a tree, then there is a unique path from $x$ to $y$.

The girth of $G$, in symbols $g(G)$, is the length of the smallest cycle in $G$. By convention, we set $g(G)=\infty$ when $G$ is a forest. The odd girth of $G, g_{\text {odd }}(G)$, is the length of the smallest odd cycle in $G$. Again $g_{\text {odd }}(G)=\infty$ if there are no odd cycles.

The degree of a vertex $x$, denoted $d_{G}(x)$ or just $d(x)$, if there is no danger of confusion, is the cardinality of the set $E_{x}=\{y \in X: x E y\}$. We also let $\Delta(G)=\sup \left\{d_{G}(x): x \in X\right\}$. If $\Delta(G) \leq \boldsymbol{\aleph}_{0}$, we say that $G$ is locally countable, and if $d_{G}(x)<\boldsymbol{\aleph}_{0}$ for all $x$, we say that $G$ is locally finite. If $\Delta(G)<\boldsymbol{\aleph}_{0}$, we say that $G$ has bounded degree.

For $A \subseteq X$ we define the induced subgraph on $A$, written $G \mid A$, to be $\left(A, E \cap A^{2}\right)$. We say that $A \subseteq X$ is independent for $G$ if $G \mid A$ is trivial, i.e., no two vertices in $A$ are adjacent.

A graph $G=(X, E)$ is bipartite if there is a partition $X=X_{1} \sqcup X_{2}$, with each $X_{i}(i=1,2)$ independent. It is well known that a graph is bipartite if it has no odd length cycles. Any acyclic graph is therefore bipartite.

The chromatic number of a graph $G$, in symbols $\chi(G)$, is the smallest cardinality of a set $Y$ for which there is a map $c: X \rightarrow Y$ (a vertex coloring) such that $x E y \Rightarrow$ $c(x) \neq c(y)$. Thus the graph is bipartite iff $\chi(G) \leq 2$.

(B) Let now $X$ be a standard Borel space. By a measure on $X$ we mean a finite Borel measure. If $\mu$ is a measure on $X$ with $0<\mu(X)<\infty$ we call the pair $(X, \mu)$ a standard measure space. If $\mu(X)=1$, we call $(X, \mu)$ a standard probability space and $\mu$ a probability measure. Unless otherwise indicated or clear from context (e.g., when $X$ is finite), measures will be assumed to be non-atomic.

(C) When $G=(X, E)$ is a graph and $X$ a standard Borel space, we say that $G$ is Borel if $E \subseteq X^{2}$ is Borel. In this situation we have that $E^{*}$ is an analytic equivalence relation, but if we assume in addition that $G$ is locally countable, then $E^{*}$ is a countable (i.e., having all its classes countable) Borel equivalence relation. We will be primarily interested in locally countable Borel graphs, and will thus borrow from the theory of countable Borel equivalence relations. For more details see, e.g., [25].

A countable Borel equivalence relation $R$ on a standard measure space $(X, \mu)$ is measure preserving, abbreviated m.p., if whenever $f: X \rightarrow X$ is a Borel automorphism with graph contained in $R, f_{*} \mu=\mu$ (where, as usual, $f_{*} \mu(A)=$ $\left.\mu\left(f^{-1}(A)\right)\right)$. For $A \subseteq X$, we define the $R$-saturation of $A$, written $[A]_{R}$, to be the set $\{x \in X: \exists y \in A(x R y)\}$. We say that $R$ is ergodic (relative to $\mu$ ) if for all Borel $A \subseteq X,[A]_{R}$ is either $\mu$-null or $\mu$-conull. When $R$ can be written as an increasing union of finite (i.e., having all its classes finite) Borel equivalence relations on $X$ we call it hyperfinite, and when $R$ admits a Borel transversal (i.e., a set meeting each $R$-class in exactly one point), we say it is smooth. Similarly, we call $R \mu$-hyperfinite 
(resp., $\mu$-smooth) if its restriction to a conull $R$-invariant Borel set is hyperfinite (resp., smooth). We say $G=(X, E)$ is m.p. if $E^{*}$ is; likewise we say that $G$ is ergodic, hyperfinite, or smooth, if $E^{*}$ is.

\section{Chromatic and independence numbers}

(A) A coloring of a locally countable Borel graph $G=(X, E)$ on a standard Borel space $X$ is a map $c: X \rightarrow Y$, where $Y$ is a standard Borel space, such that

$$
x E y \Rightarrow c(x) \neq c(y),
$$

i.e., $\forall y \in Y\left(c^{-1}(\{y\})\right.$ is independent). The chromatic number of $G$, in symbols

$$
\chi(G)
$$

is the smallest cardinality of a space $Y$ as above for which there is a coloring $c: X \rightarrow$ $Y$. Clearly $\chi(G) \in\left\{1,2, \ldots, n, \ldots, \aleph_{0}\right\}$ (and $\chi(G) \geq 2$ unless $E=\emptyset$ ).

(B) If the coloring $c$ as in (A) is Borel as a map from $X$ into $Y$, we call $c$ a Borel coloring. We define the Borel chromatic number of $G$, in symbols

$$
\chi_{B}(G),
$$

to be the smallest cardinality of a standard Borel space $Y$ for which there is a Borel coloring $c: X \rightarrow Y$. Clearly $\chi_{B}(G) \in\left\{1,2,3, \ldots, n, \ldots, \aleph_{0}, 2^{\aleph_{0}}\right\}$ and

$$
\chi(G) \leq \chi_{B}(G) .
$$

Example 2.1. In [26] and Miller [39], various examples of non-smooth $G=(X, E)$ are discussed, all of which are acyclic (so $\chi(G)=2$ ), but $\chi_{B}(G)$ ranges over all values in $\left\{2,3, \ldots, \aleph_{0}, 2^{\aleph_{0}}\right\}$. It follows that for any $m, n \in\left\{2,3, \ldots, \aleph_{0}, 2^{\aleph_{0}}\right\}$ with $m \leq n$ and $m<2^{\aleph_{0}}$, there is such a $G$ with $\chi(G)=m$ and $\chi_{B}(G)=n$ (just add a single connected component of chromatic number $m$ to a graph $G$ that has $\chi(G)=2$ and $\left.\chi_{B}(G)=n\right)$.

(C) Suppose now that $(X, \mu)$ is a standard measure space (perhaps with atoms) and $G=(X, E)$ is a locally countable Borel graph on $X$. We define the $(\mu-)$ measurable chromatic number of $G$, in symbols

$$
\chi_{\mu}(G)
$$

as the smallest cardinality of a standard Borel space $Y$ for which there is a $(\mu$-)measurable coloring $c: X \rightarrow Y$. Again,

$$
\chi(G) \leq \chi_{\mu}(G) \leq \chi_{B}(G) .
$$


Example 2.2. a) There is an acyclic, locally countable Borel graph $G$ on a standard measure space $(X, \mu)$ with $G$ m.p., ergodic and

$$
2=\chi(G)<\chi_{\mu}(G)=\chi_{B}(G)=2^{\aleph_{0}} .
$$

To see this, take a compact, metrizable group $X$ that contains a dense subset $\left\{a_{n}\right\}_{n \in \mathbb{N}}$ which generates freely a free subgroup (e.g., $X=\mathrm{SO}_{3}(\mathbb{R})$ ) and let $\mu$ be the Haar measure on $X$. Consider the graph $G=(X, E)$, where $x E y \Longleftrightarrow \exists n\left(x=a_{n}^{ \pm 1} y\right)$. Then $G$ is acyclic and m.p., ergodic. So $\chi(G)=2$ but if $c: X \rightarrow \mathbb{N}$ is a measurable coloring, then for some $n, Y=c^{-1}(\{n\})$ has positive measure, so $Y Y^{-1}$ contains an open neighborhood of 1 . Then there are $x, y \in Y, k \in \mathbb{N}$ with $x y^{-1}=a_{k}$, thus $x E y$, a contradiction.

b) Another family of examples that have the above properties are the following: Take an infinite countable group $\Gamma$ and an infinite set of generators $S$. Let $a$ be a free, mixing, measure preserving action of $\Gamma$ on $(X, \mu)$ and let $G(S, a)$ be the associated graph. Then, by the mixing property, if $A \subseteq X$ has positive measure, then for some $s \in S, s \cdot A \cap A$ has also positive measure, thus $A$ is not independent and therefore $\chi_{\mu}(G(S, a))=2^{\aleph_{0}}$. If we take $\Gamma$ to be the free subgroup on infinitely many (free) generators $S$, then $G(S, a)$ is also acyclic.

Example 2.3. There is an acyclic, locally countable Borel graph $G$ on a standard measure space $(X, \mu)$ with $G$ m.p., ergodic and

$$
2=\chi(G)<3=\chi_{\mu}(G)<\chi_{B}(G)=2^{\aleph_{0}} .
$$

Take, for instance, the graph $G_{0}$ on $X=2^{\mathbb{N}}$ defined in [26], where it is shown that $G_{0}$ is acyclic (thus $\chi\left(G_{0}\right)=2$ ), but $\chi_{B}\left(G_{0}\right)=2^{\aleph_{0}}$. Miller [39] showed that $\chi_{\mu}\left(G_{0}\right)=3$, where $\mu$ is the usual product measure on $2^{\mathbb{N}}$, for which $G_{0}$ is m.p., ergodic.

Example 2.4. It is easy to construct an example of a locally countable Borel graph $G$ on a standard measure space $(X, \mu)$ with $G$ m.p., ergodic, for which $\chi(G)=$ $\chi_{\mu}(G)=\chi_{B}(G)=2$. Take a countable, measure-preserving, ergodic equivalence relation $R$ on $(X, \mu)$ and let $X=A \sqcup B$ be a Borel partition of $X$ with $A, B$ meeting each $R$-class. Let $E$ be the bipartite graph with edges between all pairs of $R$-related points, one in $A$ and the other in $B$. Clearly $\chi(G)=\chi_{\mu}(G)=\chi_{B}(G)=2$ and $G$ generates $R$.

Example 2.5. We will see in Section 4 examples of acyclic, bounded degree Borel graphs $G=(X, E)$ on standard $(X, \mu)$ which are m.p., ergodic, and $\chi_{\mu}(G)$ is finite but arbitrarily large (although of course $\chi(G)=2$ ). From this it follows that for each $2 \leq n<\aleph_{0}$, there is an acyclic, bounded degree $G=(X, E)$ on a standard measure space $(X, \mu)$ with $G$ m.p., ergodic such that $\chi_{\mu}(G)=n$. To go from such a $G$ that has $\chi_{\mu}(G)=k+1>3$ to a $\bar{G}, \bar{\mu}$ that has $\chi_{\bar{\mu}}(\bar{G})=k$, take a partition 
$A_{0} \sqcup \cdots \sqcup A_{k}=X$ given by a measurable coloring of $G$, and assume without loss of generality that $\mu\left(A_{0}\right)<1$. Let $X^{\prime}=A_{1} \sqcup \cdots \sqcup A_{k}, G^{\prime}=G \mid X^{\prime}=\left(X^{\prime}, E^{\prime}\right)$ be the induced subgraph on $X^{\prime}$ and let $\mu^{\prime}=\mu \mid X^{\prime}$. Clearly $G^{\prime}$ is acyclic, $\chi_{\mu^{\prime}}\left(G^{\prime}\right)=k$, and $G^{\prime}$ is m.p. (for $\mu^{\prime}$ ). Consider then the ergodic decomposition associated with $\left(E^{\prime}\right)^{*}$ (on $X^{\prime}$ ). If all the pieces of this decomposition have (for the induced subgraph $\left.G^{\prime}\right)$ measurable chromatic number $\leq k-1$ then, by measurable selection, we can find a $\mu^{\prime}$-conull set on which $G^{\prime}$ admits a $k-1$ measurable coloring, and since $G^{\prime}$ has chromatic number $\leq 2$, it follows that $\chi_{\mu}\left(G^{\prime}\right) \leq k-1$, a contradiction. So there is a piece of the ergodic decomposition $(\bar{X}, \bar{\mu})$, so that if $\bar{G}=G^{\prime} \mid \bar{X}$ is the induced subgraph, then $\bar{G}$ is acyclic, $\chi_{\bar{\mu}}(\bar{G})=k$, and $\bar{G}$ is m.p., ergodic. Finally, $\bar{\mu}$ is non-atomic, else $\bar{X}$ would have to be finite, and so $\bar{G}$ would have chromatic (and so $\bar{\mu}$-measurable chromatic) number at most $2<k$, a contradiction.

An analogous argument produces, for each $2 \leq n<\boldsymbol{\aleph}_{0}$, an acyclic, bounded degree Borel graph $G$ with $\chi_{B}(G)=n$, answering a question in [39].

Example 2.6. There is an example of a locally countable Borel graph $G$ on a standard measure space $(X, \mu)$ with $G$ invariant, ergodic for which $\chi(G) \leq 3$ and $\chi_{\mu}(G)=$ $\aleph_{0}$. To see this, take, for each $n$, as in Example 2.5, an acyclic, locally countable Borel graph $G_{n}=\left(X_{n}, E_{n}\right)$ on a standard probability space $\left(X_{n}, \mu_{n}\right)$ with $G_{n}$ invariant, ergodic, and $\chi_{\mu_{n}}\left(G_{n}\right)>n$. Fix a standard probability space $(X, \mu)$ and a Borel partition $X=\bigsqcup_{n=1}^{\infty} A_{n}$ with $\mu\left(A_{n}\right)=1 / 2^{n}$. Fix a Borel bijection $\varphi_{n}: X_{n} \rightarrow A_{n}$ sending $\mu_{n}$ to $\mu_{A_{n}}=\frac{\mu \mid A_{n}}{\mu\left(A_{n}\right)}$ and let $G_{n}^{\prime}=\left(A_{n}, E_{n}^{\prime}\right)$ be the image of $G_{n}$ under this bijection. Find, for each $n$, two disjoint Borel sets $C_{n}, D_{n} \subseteq A_{n}$ of positive measure which are independent for $G_{n}^{\prime}$, and such that there are measure-preserving Borel isomorphisms $\omega_{n}: C_{n} \rightarrow C_{n+1}$, for $n \geq 1, n$ odd, and $\psi_{n}: D_{n} \rightarrow D_{n+1}$ for $n \geq 2, n$ even. Let $G=(X, E)$ be the graph on $X$ whose edges are those in $\bigcup_{n} E_{n}^{\prime}$ together with the graphs of $\omega_{n}, \psi_{n}$, and their inverses. Then $G$ is m.p., ergodic and $\chi_{\mu}(G)=\aleph_{0}$. Finally, $\chi(G) \leq 3$. Indeed, fix the same colors $a, b$ witnessing the 2-colorability of each $G_{n}^{\prime}$. Then change the color of each element of $\bigcup_{n \text {, odd }} C_{n} \cup \bigcup_{n \text {, even }} D_{n}$ to some third color $c$. This gives a 3-coloring of $G$.

We do not know an example of $G, X, \mu$ as above for which $G$ is acyclic (or even has $\chi(G)=2$ ) but $\chi_{\mu}(G)=\aleph_{0}$. (Addendum. Recently Conley-Miller [8] constructed an example of an acyclic such $G$ with $\chi_{B}(G)=\chi_{\mu}(G)=\aleph_{0}$.) More generally, we do not know what are the possible values of $k, l, m \in\left\{2,3, \ldots, \aleph_{0}, 2 \aleph_{0}\right\}$ with $k \leq l \leq m$ such that there is a locally countable Borel graph $G$ on a standard measure space $(X, \mu)$ which is m.p., ergodic, and

$$
\chi(G)=k, \chi_{\mu}(G)=l, \chi_{B}(G)=m .
$$

Remark 2.7. One can also define the ( $\mu$-)almost everywhere measurable chromatic number of $G$, in symbols $\chi_{\mu}^{\mathrm{ae}}(G)$, as the smallest cardinality of a standard Borel space $Y$ for which there is a Borel set $A \subseteq X$ with $\mu(A)=1$ and a Borel coloring 
$c: A \rightarrow Y$ of the induced subgraph $G \mid A=\left(A, E \cap A^{2}\right)$. Clearly, $\chi_{\mu}^{\text {ae }}(G) \leq \chi_{\mu}(G)$. However, if $\chi_{\mu}^{\text {ae }}(G) \geq \chi(G)$, which will be the case for most graphs that we will be interested in, then $\chi_{\mu}^{\mathrm{ae}}(G)=\chi_{\mu}(G)$.

(D) Finally, when $(X, \mu)$ is a standard measure space and $G$ is a locally countable Borel graph on $X$, we define the approximate $(\mu$-)measurable chromatic number of $G$, in symbols

$$
\chi_{\mu}^{\mathrm{ap}}(G),
$$

to be the smallest cardinality of a standard Borel space $Y$ such that for every $\varepsilon>0$ there is a Borel set $A \subseteq X$ with $\mu(X \backslash A)<\varepsilon$ and a measurable coloring $c: A \rightarrow Y$ of the induced subgraph $G \mid A$. Again,

$$
\chi_{\mu}^{\mathrm{ap}}(G) \leq \chi_{\mu}(G),
$$

but clearly $\chi(G) \leq \chi_{\mu}^{\mathrm{ap}}(G)$ may fail, since $\chi(G)$ can be altered arbitrarily on a single connected component without affecting $\chi_{\mu}^{\mathrm{ap}}(G)$. On the other hand, let

$$
\chi^{*}(G)
$$

be the minimum of all $\chi(G \mid A)$, where $A$ is an $E^{*}$-invariant Borel set of measure 1 . Clearly $\chi^{*}(G) \leq \chi(G)$. Then it is easy to see that if $G$ is m.p., then

$$
\chi^{*}(G) \leq \chi_{\mu}^{\mathrm{ap}}(G) .
$$

This is clear if $\chi_{\mu}^{\mathrm{ap}}(G) \geq \boldsymbol{\aleph}_{0}$, so assume that $\chi_{\mu}^{\mathrm{ap}}(G)=k<\boldsymbol{\aleph}_{0}$. Let $Y_{n}$ be a Borel set of measure at least $1-2^{-n}$ such that $G \mid Y_{n}$ is $k$-colorable. Let $Z_{n}=\bigcap_{m \geq n} Y_{m}$, so that $Z_{n} \subseteq Z_{n+1}$ and $\mu\left(Z_{n}\right) \geq 1-\sum_{m \geq n} 2^{-m} \rightarrow 1$, as $n \rightarrow \infty$. Then $Z \stackrel{\bigcup_{n}}{=} Z_{n}$ has measure 1 , thus contains an $E^{*}$-invariant Borel set $W \subseteq Z$ of measure 1 . To show that $G \mid W$ is $k$-colorable, it suffices to show that $G \mid F$ is $k$-colorable for every finite $F \subseteq W$, but this is clear since there must exist some $n$ such that $F \subseteq Z_{n}$.

Example 2.8. There is an acyclic, bounded degree Borel graph $G=(X, E)$ on a standard measure space $(X, \mu)$ which is m.p., ergodic, and $\chi_{\mu}^{\text {ap }}(G)<\chi_{\mu}(G)$. For instance, consider the shift $S$ on $2^{\mathbb{Z}}$ and let $X \subseteq 2^{\mathbb{Z}}$ be its aperiodic part. Let $\mu$ be the restriction of the usual product measure to $X$ (note that $\mu(X)=1$ ). Let for $x, y \in X, x E y \Longleftrightarrow x=S^{ \pm 1}(y)$. Then by Rokhlin's Lemma (see, e.g., [25], 7, 7.5), $\chi_{\mu}^{\mathrm{ap}}(G)=2$. On the other hand, $\chi_{\mu}(G)=3$. Otherwise, there is a measurable partition $X=A \sqcup B$ into independent sets. Then $S(A)=B$ and $S(B)=A$, and so $\mu(A)=\mu(B)=1 / 2$ and both $A, B$ are $S^{2}$-invariant, which is impossible as $S^{2}$ is ergodic.

We have seen in Example 2.2 examples of acyclic, locally countable, Borel $G$ on standard $(X, \mu)$ which are m.p., ergodic and have no independent sets of positive 
measure, thus $\chi_{\mu}^{\mathrm{ap}}(G)=2^{\aleph_{0}}$. It is also easy to see that there is no such $G, X, \mu$ with $\chi_{\mu}^{\mathrm{ap}}(G)=1$ (i.e., there cannot exist independent Borel sets whose measure is arbitrarily close to 1$)$. Indeed, if $G=(X, E)$ is such that $G$ is m.p., ergodic, then by the uniformization theorem for Borel sets with countable sections, there is a measurepreserving Borel bijection $\varphi: A \rightarrow B$ between Borel sets of positive measure such that $(x, \varphi(x)) \in E$, for all $x \in A$. If $\mu(A)=\mu(B)=\delta$ and $\varepsilon<\delta / 2$, there can be no independent Borel set of measure bigger than $1-\varepsilon$.

Example 2.9. There is an example of $G, X, \mu$ as in Example 2.6 with $\chi(G) \leq 3$ and $\chi_{\mu}^{\mathrm{ap}}(G)=\aleph_{0}$. The graphs $G=(X, E)$ on $(X, \mu)$ from Section 4 (mentioned previously in Example 2.5) which have arbitrarily large finite $\chi_{\mu}$ actually have arbitrary large finite $\chi_{\mu}^{\mathrm{ap}}$. Then, as in Example 2.6, this gives examples of $G$ with $\chi(G) \leq 3$ and $\chi_{\mu}^{\mathrm{ap}}(G)=\aleph_{0}$.

Again, we do not know examples of such $G$ with $\chi(G)=2$ and $\chi_{\mu}^{\mathrm{ap}}(G)=\aleph_{0}$. (Addendum. Such an example, which is actually acyclic, was constructed in ConleyMiller [8].) Also, we do not know if there are such examples for which $\chi_{\mu}^{\mathrm{ap}}(G)$ takes an arbitrary value $3 \leq k<\boldsymbol{\aleph}_{0}$.

The more general problem is again whether there is any other relationship between $\chi(G), \chi^{*}(G), \chi_{\mu}(G), \chi_{\mu}^{\mathrm{ap}}(G)$, beyond the obvious $\chi(G) \leq \chi_{\mu}(G), \chi^{*}(G) \leq$ $\chi_{\mu}^{\mathrm{ap}}(G) \leq \chi_{\mu}(G)$ for locally countable (or locally finite) Borel graphs $G$ on standard measure spaces $(X, \mu)$ which are m.p., ergodic.

(E) Let finally $G$ be a locally countable Borel graph on a standard probability space $(X, \mu)$. We define the independence number of $G$, in symbols

$$
i_{\mu}(G)
$$

by

$$
i_{\mu}(G)=\sup \{\mu(Y): Y \subseteq X \text { is an independent Borel set }\} .
$$

Clearly, we can replace "Borel" by " $(\mu-)$ measurable" in this definition. (If $\mu$ is not a probability measure we replace $\mu(Y)$ by $\mu(Y) / \mu(X)$ in the definition above.)

Obviously, $0 \leq i_{\mu}(G) \leq 1$ and $i_{\mu}(G)=0$ means that there is no positive measure independent set. We have seen in 2.2 examples of such graphs (they clearly have $\left.\chi_{\mu}(G)=2^{\aleph_{0}}\right)$. If $G=(X, E)$ is a locally countable Borel graph on a standard probability space $(X, \mu)$ with $G$ m.p., ergodic, then we have seen in (D) that $i_{\mu}(G)<$ 1 (otherwise $\chi_{\mu}^{\mathrm{ap}}(G)=1$ ).

Example 2.10. For each $0<a<1$ there is an acyclic, locally countable Borel graph $G$ on a standard probability space $(X, \mu)$ which is m.p., ergodic and $i_{\mu}(G)=a$ with the supremum being attained.

To see this, first fix an acyclic $G_{1}=\left(X_{1}, E_{1}\right)$ on $\left(X_{1}, \mu_{1}\right)$ with $G_{1}$ invariant, ergodic, $\mu_{1}\left(X_{1}\right)=1-a$, and $i_{\mu_{1}}\left(G_{1}\right)=0$. Also fix $k>\frac{a}{1-a}$. Let $X_{2}$ be an uncountable standard Borel space disjoint from $X_{1}$ and partition it into $k$ uncountable 
Borel sets: $X_{2}=A_{1} \sqcup \cdots \sqcup A_{k}$. Fix a Borel subset $Y_{1}$ of $X_{1}$, meeting each $E_{1}^{*}$-class, such that $\mu_{1}\left(Y_{1}\right)=\frac{a}{k}<1-a$. For each $1 \leq i \leq k$, let $f_{i}: Y_{1} \rightarrow A_{i}$ be a Borel bijection. Use $f_{i}$ to copy the measure $\mu_{1} \mid Y_{1}$ to $A_{i}$, say $v_{i}$, and let $\mu_{2}=\sum_{i=1}^{n} v_{i}$. Then $\mu_{2}\left(X_{2}\right)=k \cdot \frac{a}{k}=a$. Let $X=X_{1} \sqcup X_{2}, \mu=\mu_{1}+\mu_{2}$. Define the graph $G=(X, E)$ as follows: the edges of $G$ are those in $E_{1}$ together with the graph of each $f_{i}$ and its inverse. Clearly it is acyclic and it is easy to see that $G$ is m.p., ergodic. Finally, $X_{2}$ is independent for $G$ and if $A \subseteq X$ is independent Borel, then clearly $\mu\left(A \cap X_{1}\right)=0$, so $\mu(A) \leq \mu\left(X_{2}\right)=a$. So $i_{\mu}(G)=a$ and the sup is attained.

Remark 2.11. When $X$ is a finite set and $\mu$ is normalized counting measure, $i_{\mu}(G)=$ $i(G)$ is usually called the independence ratio and $\alpha(G)=$ (the maximum cardinality of an independent subset of $X$ ) is called the independence number (thus $i(G)=$ $\frac{\alpha(G)}{|X|}$ ). We will not use $\alpha(G)$ in this paper, so this should not cause any confusion.

We now have the following simple inequality:

Proposition 2.12. Let $G$ be a locally countable Borel graph on a standard probability space $(X, \mu)$ (perhaps with atoms). Then

$$
\chi_{\mu}^{\mathrm{ap}}(G) \geq \frac{1}{i_{\mu}(G)} .
$$

Proof. This is clear if $i_{\mu}(G)=0$. If $\chi_{\mu}^{\mathrm{ap}}(G)=k \in \mathbb{N}$, fix $\varepsilon>0$ and independent, pairwise disjoint Borel sets $A_{1}, \ldots, A_{k}$ with $\mu\left(\bigcup_{i=1}^{k} A_{i}\right)>1-\varepsilon$. Then

$$
k \cdot i_{\mu}(G) \geq \mu\left(\bigcup_{i=1}^{k} A_{i}\right)>1-\varepsilon,
$$

so $k>\frac{1-\varepsilon}{i_{\mu}(G)}$, and we are done.

(F) We will often be interested in locally finite graphs and, in particular, those of bounded degree, where recall that $G$ has bounded degree if

$$
\Delta(G)=\sup \left\{d_{G}(x): x \in X\right\}<\aleph_{0} .
$$

Proposition 2.13 ([26], 4.5, 4.6). If $G$ is a locally finite Borel graph, then $\chi_{B}(G) \leq$ $\aleph_{0}$. If $G$ is of bounded degree, then $\chi_{B}(G) \leq \Delta(G)+1$.

Corollary 2.14. Let $(X, \mu)$ be a standard probability space and $G=(X, E) a$ locally finite Borel graph. Then $i_{\mu}(G)>0$.

Example 2.15. For each $0<a<1$, there is a bounded degree $G=(X, E)$ on a standard probability space $(X, \mu)$ which is m.p., ergodic, such that $i_{\mu}(G)=a$ and the supremum is attained. 
To see this, let $S$ be the shift on $2^{\mathbb{Z}}$ and let $X_{1} \subset 2^{\mathbb{Z}}$ be its aperiodic part. Let $\mu_{1}$ be the restriction to $X_{1}$ of the product measure on $2^{\mathbb{Z}}$. Let $E_{1}$ be the union of the graph of $S \mid X_{1}$ and its inverse. This is invariant, ergodic on $\left(X_{1}, \mu_{1}\right)$. Let $n>3$ be such that $a \in\left[\frac{1}{n}, \frac{n-1}{n}\right]$, so that $a=\frac{1}{n} \alpha+\frac{n-1}{n} \beta$ for some $\alpha, \beta \geq 0$, $\alpha+\beta=1$. Let $A \sqcup B=X_{1}$ be a Borel partition with $\mu_{1}(A)=\alpha, \mu_{1}(B)=\beta$. Let $X=X_{1} \times\{1, \ldots, n\}$ and give $X$ the product measure $\mu=\mu_{1} \times v$, where $v$ is the normalized counting measure on $\{1, \ldots, n\}$. Let $G=(X, E)$ be the following graph on $X$ :

$$
\begin{aligned}
E=\{ & \left.((x, 1),(y, 1)): x E_{1} y\right\} \\
& \cup\{((x, i),(x, j)): x \in A, 1 \leq i \neq j \leq n\} \\
& \cup\{((x, 1),(x, j)): x \in B, 2 \leq j \leq n\} \\
& \cup\{((x, j),(x, 1)): x \in B, 2 \leq j \leq n\} .
\end{aligned}
$$

Clearly, $d(G) \leq n+1$. It is easy to see that $(x, i) E^{*}(y, j) \Longleftrightarrow x E_{1}^{*} y$, and thus $G$ is m.p., ergodic. We claim that $i_{\mu}(G)=a$ and the supremum is attained. First note that if $Y \subseteq X$ is independent, then for each $x \in A$ there is at most one $1 \leq i \leq n$ with $(x, i) \in Y$ and for each $x \in B$ there are at most $n-1$ many $1 \leq j \leq n$ with $(x, j) \in Y$. Thus $\mu(Y) \leq \frac{1}{n} \mu(A)+\frac{n-1}{n} \mu(B)=a$. On the other hand, $Y=\{(x, 2): x \in A\} \cup\{(x, j): x \in B$ and $j \in\{2, \ldots, n\}\}$ is independent and $\mu(Y)=\frac{1}{n} \mu(A)+\frac{n-1}{n} \mu(B)=a$, so $i_{\mu}(G)=a$ and the supremum is attained.

We do not know however if examples as in 2.15 with arbitrary $i_{\mu}(G)=a \in(0,1)$ can be found which are acyclic, even if we replace "bounded degree" by "locally finite." The referee pointed out that for any given integer $d>2$, there is an upper bound $f(d)=\frac{d}{d+1}<1$ for the independence number $i_{\mu}(G)$ of every m.p., ergodic $G$ with $\Delta(G) \leq d$. This is because if $A$ is independent and $B$ is its complement, then the measure of $A$ is bounded by the integral of $d(x)$ over $B$. We will see in Section 3 that actually for $d=2, f(2)=1 / 2$ works. Note that in the examples of 2.15 , to achieve $i_{\mu}(G)=a<1$, we needed a graph $G$ of degree $n+1$, where $n \geq \frac{1}{1-a}$.

We now prove a strengthening of 2.13 with applications in computing bounds for approximate chromatic numbers (and thus independence numbers). Recall that we say a graph is aperiodic if all of its connected components are infinite.

Proposition 2.16. Suppose that $G$ is an aperiodic Borel graph on $X$ with degree bounded by $d$. Then there is a decreasing sequence $A_{1} \supseteq A_{2} \supseteq \cdots$ of subsets of $X$ with $\bigcap_{n} A_{n}=\emptyset$ and Borel $(d+1)$-colorings $c_{n}: X \rightarrow\{0,1, \ldots, d\}$ of $G$ with $c_{n}^{-1}(0) \subseteq A_{n}$.

Proof. Recall that a complete section or marker set for an equivalence relation is one meeting each equivalence class (see, e.g., [25], 6.7). We now say that a subset $A$ of $X$ is a strong marker set for $G$ if it meets every connected component, is independent, and there is some natural number $k$ such that every point of $X$ is connected to a 
point in $A$ via a path in $G$ of length less than $k$. The next lemma is a variation of Lemma 3.14 in [20].

Lemma 2.17. Suppose that $G$ is a locally finite, aperiodic Borel graph on $X$. Then there is a decreasing sequence of Borel strong marker sets $A_{1} \supseteq A_{2} \supseteq \cdots$ with $\bigcap_{n} A_{n}=\emptyset$.

Proof. We let $B_{1}$ be a maximal independent Borel set (see [26], 4.2, 4.5). We then let $B_{2}$ be a maximal Borel subset of $B_{1}$ subject to the constraint that no two points of $B_{2}$ are within distance two in the graph metric of $G$ (the existence of such $B_{2}$ follows from the fact that the distance two graph is locally finite). We continue in this fashion, letting $B_{n+1}$ be a maximal Borel subset of $B_{n}$ with no two points within distance $n+1$.

We claim that every point of $X$ is within distance $n^{2}$ of $B_{n}$. This is a simple induction. If every point $x$ is within distance $n^{2}$ of $B_{n}$, then, by maximality of $B_{n+1}$, every point of $B_{n}$ is within distance $n+1$ of $B_{n+1}$ and thus $x$ is within distance $n^{2}+n+1<(n+1)^{2}$ of $B_{n}$.

It may not be the case that $\bigcap_{n} B_{n}=\emptyset$, but this intersection meets each connected component of $G$ it at most one point. Since $G$ is aperiodic, each set $A_{n}=B_{n} \backslash \bigcap_{n} B_{n}$ is still a strong marker set. The sequence $\left(A_{n}\right)$ is as desired.

Lemma 2.18. Suppose that $G$ is a Borel graph on $X$ and that $A \subseteq X$ is a Borel set such that every point in $X \backslash A$ has degree less than $d$. Then any Borel $d$-coloring $c: A \rightarrow d$ of $G \mid A$ may be extended to a Borel $d$-coloring $c^{\prime}: X \rightarrow d$ of $G$.

Proof. Partition $X \backslash A=B_{1} \sqcup B_{2} \sqcup \cdots \sqcup B_{d}$ into $G$-independent Borel sets. Extend $c$ to $c_{1}: A \cup B_{1} \rightarrow d$ by following a greedy algorithm, i.e., for each $x \in B_{1} \operatorname{set} c_{1}(x)$ to be the least color not used by a neighbor of $x$. Similarly extend to $B_{2}, \ldots, B_{d}$.

To finish the proof of the proposition, we take the vanishing sequence of strong markers $A_{1} \supseteq A_{2} \supseteq \cdots$ granted by Lemma 2.17 as the sequence in the statement of the lemma, and describe how to Borel $(d+1)$-color $G$ with one color contained in $A_{n}$. Fix $k$ such that every point of $X$ is within distance $k$ of $A_{n}$, and partition $X$ into $X_{0}=A_{n} \sqcup X_{1} \sqcup \cdots \sqcup X_{k}$, where $X_{i}=\left\{x \in X: \rho_{G}\left(x, A_{n}\right)=i\right\}$.

Now $G \mid X_{k}$ has degree bounded by $d-1$ (since everything is connected to at least one point in $\left.X_{k-1}\right)$, and thus admits a Borel $d$-coloring. In the graph $G \mid\left(X_{k} \cup X_{k-1}\right)$, points in $X_{k-1}$ have degree bounded by $d-1$ and so Lemma 2.18 allows us to extend the $d$-coloring on $X_{k}$ to one on $X_{k} \cup X_{k-1}$. Continuing in this fashion, we obtain a Borel coloring of $X \backslash A_{n}$ with $d$ colors. Using the remaining color on $A_{n}$ itself completes the proof.

And now, for the promised application, an analogue of Brooks' Theorem. Recall that the clique number of a graph, $\operatorname{clq}(G)$, is the largest cardinality of a complete (induced) subgraph of $G$. 
Theorem 2.19. Let $(X, \mu)$ be a standard probability space, $G=(X, E)$ a Borel graph on $X$ with degree bounded by $d$, where $d \geq 3$. Suppose furthermore that $\operatorname{clq}(G) \leq d$. Then $\chi_{\mu}^{\mathrm{ap}}(G) \leq d$ and thus $i_{\mu}(G) \geq 1 / d$.

Proof. Brooks' Theorem allows us to $d$-color the finite components of $G$ in a Borel fashion: indeed, whenever $G=(X, E)$ is a Borel graph with finite connected components, then $\chi(G)=\chi_{B}(G)$. To see this, simply choose a Borel transversal $T$ of $E^{*}$. Since each $x \in T$ sees only finitely many ways (but at least one) of coloring its connected component using the colors $\{1,2, \ldots, \chi(G)\}$, the required coloring is granted by the uniformization theorem for Borel sets with countable sections.

So, it remains to handle the infinite connected components of $G$. Fix $\varepsilon>0$. Since the sequence $A_{1} \supseteq A_{2} \supseteq \cdots$ granted by Proposition 2.16 vanishes, we may fix $n$ such that $\mu\left(A_{n}\right)<\varepsilon$. But then 2.16 provides us with the required $d$-coloring of $X \backslash A_{n}$.

The analogy extends also to the case $d=2$ :

Theorem 2.20. Let $(X, \mu)$ be a standard probability space, $G=(X, E)$ a bipartite Borel graph on $X$ with degree bounded by 2. Then $\chi_{\mu}^{\mathrm{ap}}(G) \leq 2$ and thus $i_{\mu}(G) \geq 1 / 2$.

Proof. Fix $\varepsilon>0$. Then by the marker lemma (see, e.g., [25], 6.7) there is a Borel set $A$ with $\mu(A)<\varepsilon$ meeting every infinite $E^{*}$-class. The connected components of $G \mid(X \backslash A)$ are either finite or infinite with a single endpoint of degree one. This easily implies that there is a Borel 2-coloring of $G \mid(X \backslash A)$.

In some cases, the conclusion of Theorem 2.19 can be slightly improved.

Proposition 2.21. Let $(X, \mu)$ be a standardprobability space, $G=(X, E)$ a bipartite Borel graph on $X$ with degree bounded by $d$, where $d \geq 3$. Then there is an independent Borel set of measure $\geq 1 / d$.

Proof. Let $A$ be a maximal independent Borel set. If $\mu(A) \geq 1 / d$ we are done. Else, $1-\mu(A)>1-1 / d$ and so we can choose $\varepsilon>0$ small enough so that $(1-\mu(A))\left(\frac{1}{d-1}-\varepsilon\right) \geq \frac{1}{d}$. Since $G \mid(X \backslash A)$ has degree bounded by $d-1 \geq 2$, by 2.19 or 2.20 there is an independent subset of $X \backslash A$ with measure at least

$$
\mu(X \backslash A)\left(\frac{1}{d-1}-\varepsilon\right),
$$

so of measure at least $1 / d$.

Proposition 2.22. Let $(X, \mu)$ be a standard probability space, $G=(X, E)$ a Borel graph on $X$ with degree bounded by $d$, where $d \geq 4$. Suppose further that $\operatorname{clq}(G) \leq$ $d-1$. Then there is an independent Borel set of measure $\geq 1 / d$. 
Proof. Essentially the same argument as 2.21, noting that the assumption of small clique number allows us to always apply 2.19 in finding the large independent subset of $X \backslash A$.

\section{Hyperfinite graphs}

(A) Recall that a countable Borel equivalence relation $R$ on a standard Borel space $X$ is called hyperfinite if it can be written as an increasing union $\bigcup_{n=1}^{\infty} F_{n}$, with each $F_{n}$ a finite Borel equivalence relation. If instead $R$ is on a standard measure space $(X, \mu)$, we say that $R$ is $\mu$-hyperfinite if there is a conull Borel set $A \subseteq X$ such that $R \mid A$ is hyperfinite. By Connes-Feldman-Weiss (see, e.g., [25], 10.1), measure-preserving actions of amenable groups give rise to $\mu$-hyperfinite orbit equivalence relations. We will examine such actions in Section 4.

(B) We say that a locally countable Borel graph $G=(X, E)$ on a standard measure space $(X, \mu)$ is $\mu$-hyperfinite if the equivalence relation $E^{*}$ is $\mu$-hyperfinite. In Miller [39] it is shown that if $G$ is $\mu$-hyperfinite and acyclic, then $\chi_{\mu}(G) \leq 3$. A slight modification of these techniques allows us to compute $\chi_{\mu}^{\mathrm{ap}}(G)$ for such graphs.

We say that a locally countable Borel graph $G=(X, E)$ is smooth if $E^{*}$ admits a Borel selector. Such a graph $G$ is directable if there exists a Borel function $f: X \rightarrow$ $X$ such that $x E y \Longleftrightarrow y=f(x)$ or $x=f(y)$. Finally, such a graph is essentially linear if there is a Borel set $B \subseteq X$ such that every connected component of $G$ contains exactly one connected component of $G \mid B$ and, moreover, $G \mid B$ is an acyclic graph which is regular of degree two (i.e., it is a forest of lines).

Theorem 3.1. Let $G$ be a locally countable, acyclic, $\mu$-hyperfinite Borel graph on a standard probability space $(X, \mu)$. Then $\chi_{\mu}^{\mathrm{ap}}(G) \leq 2$, and thus $i_{\mu}(G) \geq 1 / 2$.

Proof. Following [39], 3.1, and [20], 3.19, we may find pairwise disjoint, $E^{*}$-invariant Borel sets $X_{0}, X_{1}, X_{2}$ such that $\mu\left(X_{0} \cup X_{1} \cup X_{2}\right)=1, G \mid X_{0}$ is smooth, $G \mid X_{1}$ is directable, and $G \mid X_{2}$ is essentially linear. We handle these three parts separately.

Fix a Borel transversal $A$ of $E^{*} \mid X_{0}$, and color each point $x \in X_{0}$ by the parity of $\rho_{G}(x, A)$. Thus, $\chi_{B}\left(G \mid X_{0}\right) \leq 2$, and consequently $\chi_{\mu}^{\mathrm{ap}}\left(G \mid X_{0}\right) \leq 2$.

We next handle $X_{2}$. Fix $\varepsilon>0$ and a Borel set $B$ witnessing the essential linearity of $G \mid X_{2}$. By Theorem 2.20, we may find a Borel partition $B=B_{0} \sqcup B_{1} \sqcup B_{2}$, with $\mu\left(B_{2}\right)<\varepsilon$ and $B_{0}, B_{1}$ forming a 2-coloring of $G \mid\left(B \backslash B_{2}\right)$ (if $\mu(B)=0$ we may take $\left.B_{2}=B\right)$. We may extend this to a 2-coloring of $G \mid\left(X_{2} \backslash B_{2}\right)$ in the obvious way: for each $x \in X_{2}$ set $b(x)$ to be the closest element of $B$ to $x$, then color $x$ by the parity of $\rho_{G}(x, b(x))$ if $b(x) \in B_{0}$ and by the parity of $\rho_{G}(x, b(x))+1$ if $b(x) \in B_{1} \cup B_{2}$. Thus, $\chi_{\mu}^{\text {ap }}\left(G \mid X_{2}\right) \leq 2$.

Finally, we handle $X_{1}$. Fix $\varepsilon>0$ and a Borel function $f: X_{1} \rightarrow X_{1}$ witnessing that $G \mid X_{1}$ is directable. Define a partial order on $X_{1}$ by $x \leq y \Longleftrightarrow \exists n\left(y=f^{n}(x)\right)$. 
The following generalization of the marker lemma ensures that we may find small sets cofinal in this partial order. For a relation $R$ on $X$, we say that $A \subseteq X$ is an $R$-complete section if $A$ meets every vertical section of $R$, i.e., for all $x$ in $X$, $\exists y \in A(x R y)$.

Lemma 3.2 (Miller). Suppose that $X$ is a Polish space and $R$ is a transitive, reflexive Borel binary relation on $X$ whose vertical sections are all countably infinite. Then there are Borel $R$-complete sections $A_{0} \supseteq A_{1} \supseteq \cdots$ such that $\bigcap_{n \in \mathbb{N}} A_{n}=\emptyset$.

Using the above lemma, we may find a Borel set $C \subseteq X_{1}$ with $\mu(C)<\varepsilon$ so that for all $x \in X_{1}$ there exists $y \in C$ with $x \leq y$. We may then color each $x \in X_{1} \backslash C$ by the parity of the least $n$ such that $f^{n}(x) \in C$, so $\chi_{\mu}^{\text {ap }}\left(G \mid X_{1}\right) \leq 2$.

Proof of Lemma 3.2. Fix an enumeration $B_{0}, B_{1}, \ldots$ of a countable family of Borel subsets of $X$ which separates points, and for each $s \in 2^{<\mathbb{N}}$, define $B_{s} \subseteq X$ by

$$
B_{s}=\left(\bigcap_{s(i)=0} X \backslash B_{i}\right) \cap\left(\bigcap_{s(i)=1} B_{i}\right) .
$$

For each $n \in \mathbb{N}$, define $S_{n}: X \rightarrow \mathcal{P}\left(2^{n}\right)$ by

$$
S_{n}(x)=\left\{s \in 2^{n}: \forall y \in R_{x}\left(\left|B_{s} \cap R_{y}\right|=\aleph_{0}\right)\right\} .
$$

Sublemma 3.3. Suppose that $x, y \in X, n \in \mathbb{N}, s \in 2^{n}$, and $i \in\{0,1\}$. Then:

(1) $(x, y) \in R \Longrightarrow S_{n}(x) \subseteq S_{n}(y)$;

(2) $s i \in S_{n+1}(x) \Longrightarrow s \in S_{n}(x)$.

Proof. The first claim is a consequence of the transitivity of $R$, and the second is a trivial consequence of the definition of $S_{n}$.

For each $s \in 2^{n}$, define $C_{s} \subseteq X$ by

$$
C_{s}=\left\{x \in X: \forall y \in R_{x}\left(s=\min _{\operatorname{lex}} S_{n}(y)\right)\right\},
$$

and for each $n \in \mathbb{N}$, define $D_{n} \subseteq X$ by

$$
D_{n}=\bigcup_{s \in 2^{n}} B_{s} \cap C_{s}
$$

We will show that the sets $D_{0}, D_{1}, \ldots$ are nearly as desired.

Sublemma 3.4. $\forall n \in \mathbb{N}\left(D_{n+1} \subseteq D_{n}\right)$. 
Proof. Fix $n \in \mathbb{N}$ and suppose that $x \in D_{n+1}$. Then there exists $s \in 2^{n}$ and $i \in\{0,1\}$ such that $x \in B_{s i} \cap C_{s i}$. In particular, it follows that $x \in B_{s}$, so to see that $x \in D_{n}$, it is enough to show that $x \in C_{s}$. Suppose, towards a contradiction, that there exists $y \in R_{x}$ such that $s \neq t$, where $t=\min _{\text {lex }} S_{n}(y)$. As $x \in C_{s i}$, it follows that si $\in S_{n+1}(x)$. As (1) ensures that $S_{n}(x) \subseteq S_{n}(y)$ and (2) ensures that $s \in S_{n}(x)$, it follows that $s \in S_{n}(y)$, thus $t<_{\text {lex }} s$. As $t 0<_{\operatorname{lex}} s i$ and $s i=\min _{\text {lex }} S_{n+1}(y)$, it follows that $t 0 \notin S_{n+1}(y)$, so there exists $z \in R_{y}$ such that $\left|B_{t 0} \cap R_{z}\right|<\aleph_{0}$. Similarly, since $t 1<_{\text {lex }} s i$ and $s i=\min _{\text {lex }} S_{n+1}(z)$, it follows that $t 1 \notin S_{n+1}(z)$, so there exists $w \in R_{z}$ such that $\left|B_{t 1} \cap R_{w}\right|<\boldsymbol{\aleph}_{0}$. As the transitivity of $R$ ensures that $R_{w} \subseteq R_{z}$, this implies that $\left|B_{t} \cap R_{w}\right|<\boldsymbol{\aleph}_{0}$. As the transitivity of $R$ implies also that $(y, w) \in R$, this contradicts our assumption that $t \in S_{n}(y)$.

While each $D_{n}$ is an $R$-complete section, we will show something stronger:

Sublemma 3.5. $\forall x \in X \forall n \in \mathbb{N}\left(\left|D_{n} \cap R_{x}\right|=\aleph_{0}\right)$.

Proof. Fix an enumeration $\left\langle s_{i}\right\rangle_{i<2^{n}}$ of $\{0,1\}^{n}$. For each $x \in X$, set $x_{0}=x$, and given $x_{i}$, let $x_{i+1}$ be any element of $R_{x_{i}}$ such that $\min _{\text {lex }} S_{n}\left(x_{i+1}\right) \neq \min _{\text {lex }} S_{n}\left(x_{i}\right)$, if such an element exists. Otherwise, set $x_{i+1}=x_{i}$. Let $y=x_{2^{n}}$ and $s=\min _{\text {lex }} S_{n}(y)$, and observe that $\forall z \in R_{y}\left(s=\min _{\text {lex }} S_{n}(z)\right)$, thus $y \in C_{s}$. As $s \in S_{n}(y)$, it follows that $\left|B_{s} \cap R_{y}\right|=\aleph_{0}$, and since $y \in C_{s}$, it follows that $B_{s} \cap R_{y}=B_{s} \cap C_{s} \cap R_{y}$, thus $\left|B_{s} \cap C_{s} \cap R_{y}\right|=\aleph_{0}$. As $B_{s} \cap C_{s} \subseteq D_{n}$ and the transitivity of $R$ ensures that $R_{y} \subseteq R_{x}$, it follows that $\left|D_{n} \cap R_{x}\right|=\aleph_{0}$.

Unfortunately, it need not be the case that the set $D=\bigcap_{n \in \mathbb{N}} D_{n}$ is empty. However, this is not so far from the truth:

Sublemma 3.6. $\forall x, y \in D(x \neq y \Longrightarrow(x, y) \notin R)$.

Proof. Suppose, towards a contradiction, that there are distinct points $x, y \in D$ such that $(x, y) \in R$. Fix $n \in \mathbb{N}$ and $s \in 2^{n}$ such that $x \in B_{s}$ and $y \notin B_{s}$. As $x \in D_{n}$, it follows that $\min _{\text {lex }} S_{n}(x)=\min _{\text {lex }} S_{n}(y)=s$, so $y \notin D_{n}$, thus $y \notin D$, the desired contradiction.

Now define $A_{n}=D_{n} \backslash D$. Sublemma 3.4 implies that these sets are decreasing, and they clearly have empty intersection, so it only remains to check that each $A_{n}$ is an $R$-complete section. Towards this end, fix $x \in X$, and observe that two applications of Sublemma 3.5 ensure that there are distinct points $y \in D_{n} \cap R_{x}$ and $z \in D_{n} \cap R_{y}$. Sublemma 3.6 then ensures that $y \notin A_{n} \Rightarrow y \in D \Rightarrow z \notin D \Rightarrow z \in A_{n}$, and the transitivity of $R$ then implies that $A_{n} \cap R_{x} \neq \emptyset$.

It is natural to ask whether the assumption of acyclicity in Theorem 3.1 can be weakened to $\chi(G) \leq 2$. This is not the case: 
Example 3.7. There is a Borel graph $G$ on a standard probability space $(X, \mu)$ which is locally countable, $\mu$-hyperfinite, and satisfies both $\chi(G)=2$ and $i_{\mu}(G)=0$. To see this, set $X=2^{\mathbb{N}}, \mu$ the usual product measure, and $x E y \Longleftrightarrow x$ and $y$ differ on exactly one coordinate. Then $E^{*}=E_{0}$, the equivalence relation of eventual agreement, which is hyperfinite. Certainly, $\chi(G)=2$ as $G$ contains no cycles of odd length. It remains only to see that $i_{\mu}(G)=0$.

Suppose that $Y \subseteq X$ is a set of positive measure. Then, by Lebesgue density, there is a finite binary string $s$ such that $\frac{\mu\left(Y \cap \mathcal{N}_{s}\right)}{\mu\left(\mathcal{N}_{s}\right)}>1 / 2$, where $\mathcal{N}_{s}=\{x \in X: s \sqsubseteq x\}$. This implies that the set $\{x \in X: s 0 x \in Y$ and $s 1 x \in Y\}$ has positive measure, so $Y$ cannot be independent for $G$.

(C) We now turn our attention to locally finite $\mu$-hyperfinite graphs. In this context, counterexamples such as 3.7 do not arise:

Theorem 3.8. Let $G$ be a locally finite, $\mu$-hyperfinite Borel graph on a standard probability space $(X, \mu)$. Then $\chi_{\mu}^{\mathrm{ap}}(G) \leq \chi^{*}(G)$, and thus $i_{\mu}(G) \geq 1 / \chi^{*}(G)$. If moreover $G$ is m.p., then $\chi_{\mu}^{\mathrm{ap}}(G)=\chi^{*}(G)$.

Proof. For the first part, discarding a null set if necessary, we may assume that $G$ is hyperfinite and $\chi^{*}(G)=\chi(G)$. Fix $\varepsilon>0$ and finite Borel equivalence relations $F_{1} \subseteq F_{2} \subseteq \cdots \subseteq F_{n} \subseteq \cdots$ witnessing the hyperfiniteness of $G$. For each $n$, define the set

$$
X_{n}=\left\{x \in X: \forall y \in X\left(x E y \Longrightarrow x F_{n} y\right)\right\} .
$$

Then, since $G$ is locally finite, $X=\bigcup_{n} X_{n}$. Choose $n$ such that $\mu\left(X_{n}\right)>1-\varepsilon$, and define $G^{\prime}=G \mid X_{n}=\left(X_{n}, E^{\prime}\right)$. Since $E^{\prime} \subseteq F_{n}$, we have $\left(E^{\prime}\right)^{*} \subseteq F_{n}$, and thus the connected components of $G^{\prime}$ are finite. Consequently $\chi_{B}\left(G^{\prime}\right) \leq \chi\left(G^{\prime}\right) \leq \chi(G)$, and therefore $\chi_{\mu}^{\mathrm{ap}}(G) \leq \chi(G)$.

In the m.p. case, we always have $\chi^{*}(G) \leq \chi_{\mu}^{\mathrm{ap}}(G)$, thus we have equality.

\section{Graphs associated with group actions}

(A) Consider a countable group $\Gamma$, which we will assume to be infinite, unless otherwise indicated. Let $A: \Gamma \times X \rightarrow X$ be a free Borel action of $\Gamma$ on a standard Borel space. (Free means that $A(\gamma, x) \neq x, \forall \gamma \neq 1, \forall x \in X$.) We put $\gamma^{A}(x)=A(\gamma, x)$ and often write $\gamma \cdot x$ for $A(\gamma, x)$ if there is no danger of confusion.

If $S \subseteq \Gamma$ is a set of generators for $\Gamma$, where we always assume, unless otherwise indicated, that $1 \notin S$, we define the associated graph

$$
G(S, A)=(X, E(S, A))
$$

by

$$
(x, y) \in E(S, A) \Longleftrightarrow \exists s \in S\left(y=s^{ \pm 1} \cdot x\right) .
$$


Clearly this is a locally countable Borel graph on $X$ whose connected components are the $\Gamma$-orbits of the action $A$. Denote by $\chi(S, A)$, resp., $\chi_{B}(S, A)$ the associated with $G(S, A)$ chromatic, resp., Borel chromatic numbers. Let also Cay $(\Gamma, S)$ be the (left) Cayley graph of $\Gamma$ with respect to $S$, where $\gamma, \delta$ are connected by an edge iff there is $s \in S$ with $\delta=s^{ \pm 1} \gamma$. If $x \in X$ then the map $\gamma \in \Gamma \mapsto \gamma \cdot x$ gives an isomorphism between $\operatorname{Cay}(\Gamma, S)$ and the connected component of $x$ in $G(S, A)$. Thus

$$
\chi(S, A)=\chi(\operatorname{Cay}(\Gamma, S)) .
$$

Let now $(X, \mu)$ be a standard probability space and let $a$ be a free, measure-preserving action of $\Gamma$ on $(X, \mu)$. This is an equivalence class of Borel actions of $\Gamma$ on $X$ that preserve the measure $\mu$, where two actions $A(\gamma, x), B(\gamma, x)$ are identified if $A(\gamma, x)=$ $B(\gamma, x)$, a.e., $\forall \gamma$, and free means that $\forall \gamma \neq 1(a(\gamma, x) \neq x$, a.e. $)$. We again denote, for each set $S$ of generators of $\Gamma$, by $G(S, a)=(X, E(S, a))$ the associated graph on $X$ and by $\chi^{*}(S, a), \chi_{\mu}(S, a), \chi_{\mu}^{\mathrm{ap}}(S, a), i_{\mu}(S, a)$ the corresponding numbers. It should be noted that $G(S, a)$ is only defined almost everywhere in the sense that if $A, B$ are representatives for $a$, then there is a conull set $Y$ which is invariant under both $A$ and $B$ on which $A$ and $B$ agree, thus $Y$ is a set of connected components of both $G(S, A), G(S, B)$ and $G(S, A)|Y=G(S, B)| Y$. It follows that the numbers $\chi^{*}, \chi_{\mu}, \chi_{\mu}^{\mathrm{ap}}, i_{\mu}$ are well defined, i.e., depend only upon $a$ (and $S$ ). Moreover, again, $\chi^{*}(S, a)=\chi(\operatorname{Cay}(\Gamma, S)) \leq \chi_{\mu}^{\mathrm{ap}}(S, a)$. Finally, we usually write $E_{a}$ for $E^{*}(S, a)$, the equivalence relation induced by the action $a$. Clearly, $G(S, a)$ is measure preserving and it is ergodic iff $a$ is ergodic.

We first note the following obvious inequality

$$
i_{\mu}(S, a) \leq 1 / 2 .
$$

This is because if $A \subseteq X$ is independent and $s \in S$, then $A \cap s^{a}(A)=\emptyset$ and $\mu(A)=\mu\left(s^{a}(A)\right)$.

Moreover, if for every $\Gamma_{0} \leq \Gamma$ of index at most 2 , the action $a \mid \Gamma_{0} \in A\left(\Gamma_{0}, X, \mu\right)$ is ergodic (e.g., if $a$ is weakly mixing), then there can be no independent Borel set $A$ of measure exactly $1 / 2$ (so if $i_{\mu}(S, a)=1 / 2$, the supremum is not attained). Otherwise $s \cdot A=X \backslash A$ for every $s \in S^{ \pm 1}$ and so $A$ is $\Gamma_{0}$-invariant, where $\Gamma_{0}=\left\{t_{1} t_{2} \ldots t_{2 n}: n \geq 0, t_{i} \in S^{ \pm 1}\right\}$ and $S^{ \pm 1}=\left\{s^{ \pm 1}: s \in S\right\}$. Since $\left[\Gamma: \Gamma_{0}\right] \leq 2$, this gives a contradiction to our assumption. In particular, for such $a, \chi_{\mu}(S, a) \geq 3$.

Finally we note that by 2.2 , (b) if $S$ is an infinite set of generators and $a \in$ $\operatorname{FR}(\Gamma, X, \mu)$ is mixing, then $i_{\mu}(S, a)=0$.

We denote by $\operatorname{FR}(\Gamma, X, \mu)$ the space of free, measure-preserving actions of $\Gamma$ on $(X, \mu)$ and we equip it with the weak topology in which $\operatorname{FR}(\Gamma, X, \mu)$ is a Polish space (see [23], 10).

Theorem 4.1. Let $\Gamma$ be a countable group and $S \subseteq \Gamma$ a finite set of generators. Then the map

$$
a \mapsto i_{\mu}(S, a)
$$

is lower semicontinuous in $\mathrm{FR}(\Gamma, X, \mu)$. 
Proof. Note that for $r \in \mathbb{R}$,

$$
\begin{aligned}
r<i_{\mu}(S, a) \Longleftrightarrow & \exists \text { Borel } A \exists \varepsilon>0 \\
& \left(\mu(A)>r+\varepsilon \text { and } \forall t \in S^{ \pm 1}\left(\mu\left(A \cap t^{a}(A)\right)<\frac{\varepsilon}{n}\right)\right),
\end{aligned}
$$

where $\left|S^{ \pm 1}\right|=n$. The direction from left to right is clear, because we can take $A$ to be an independent Borel set of measure $r+\varepsilon$ for some $\varepsilon>0$. Conversely, let $A, \varepsilon$ satisfy the right-hand side. Then

$$
B=A \backslash \bigcup_{t \in S^{ \pm 1}} t^{a}(A)
$$

is independent and $\mu(B) \geq \mu(A)-n \cdot \frac{\varepsilon}{n}>r$, so $i_{\mu}(S, a)>r$.

Since the map $a \mapsto \mu\left(A \cap \gamma^{a}(A)\right)$ from $\operatorname{FR}(\Gamma, X, \mu)$ to $\mathbb{R}$ is continuous in the weak topology, for each $\gamma \in \Gamma,\left\{a \in \operatorname{FR}(\Gamma, X, \mu): r<i_{\mu}(S, a)\right\}$ is open and the proof is complete.

Recall that $a \in \operatorname{FR}(\Gamma, X, \mu)$ is weakly contained in $b \in \mathrm{FR}(\Gamma, X, \mu)$, in symbols $a \prec b$, if $a$ is in the closure of the conjugacy class of $b$ (see [23], 10, 10.1). So we have

Corollary 4.2. Let $\Gamma$ be a countable group and $S \subseteq \Gamma$ a finite set of generators. Then

$$
a \prec b \Rightarrow i_{\mu}(S, a) \leq i_{\mu}(S, b) .
$$

In particular, $i_{\mu}(S, a)$ is an invariant of weak equivalence, defined by $a \sim b \Longleftrightarrow$ $a \prec b$ and $b \prec a$.

Corollary 4.2 (and thus 4.1) may fail if $S$ is infinite. Take for example the free group $\Gamma$ with a (free) infinite generating set $S$. Then every action of $\Gamma$ is weakly contained in a mixing action (this is a very special case of the result in [16]), so by 2.2 (b) if 4.2 was true in this case, then we would have $i_{\mu}(S, a)=0$, for any free action $a$. But this contradicts, for example, 4.6 below. A similar remark applies to 4.3 and 4.13.

Concerning $\chi_{\mu}^{\mathrm{ap}}$ we have the following result.

Theorem 4.3. Let $\Gamma$ be a countable group and $S \subseteq \Gamma$ a finite set of generators. Then for any $a, b \in \mathrm{FR}(\Gamma, X, \mu)$,

$$
a \prec b \Longrightarrow \chi_{\mu}^{\mathrm{ap}}(S, a) \geq \chi_{\mu}^{\mathrm{ap}}(S, b) .
$$

Proof. Assume that $a \prec b$, and let $k=\chi_{\mu}^{\mathrm{ap}}(S, a)$ and $n=\left|S^{ \pm 1}\right|$. Fix $\varepsilon>0$. Then consider pairwise disjoint, independent Borel subsets $A_{1}, \ldots, A_{k}$ of $X$ with 
$\mu\left(A_{1} \cup \cdots \cup A_{k}\right)>1-\frac{\varepsilon}{k+1}$. Since $a \prec b$, there are Borel, pairwise disjoint subsets $B_{1}, \ldots, B_{k}$ of $X$ with $\mu\left(B_{1} \cup \cdots \cup B_{k}\right)>1-\frac{\varepsilon}{k+1}$ and

$$
\left|\mu\left(s^{a}\left(A_{i}\right) \cap A_{i}\right)-\mu\left(s^{b}\left(B_{i}\right) \cap B_{i}\right)\right|<\frac{\varepsilon}{n(k+1)}
$$

for all $s \in S^{ \pm 1}$, thus $\mu\left(s^{b}\left(B_{i}\right) \cap B_{i}\right)<\frac{\varepsilon}{n(k+1)}$ for all $s \in S^{ \pm 1}$. If $\bar{B}_{i}=B_{i} \backslash$ $\bigcup_{s \in S^{ \pm 1}} s^{b}\left(B_{i}\right)$, then $\bar{B}_{i}, 1 \leq i \leq k$, are Borel, pairwise disjoint, independent (for the action $b)$ sets, and $\mu\left(\bar{B}_{i}\right) \geq \mu\left(B_{i}\right)-\frac{\varepsilon}{k+1}$, so $\mu\left(\bar{B}_{1} \cup \cdots \cup \bar{B}_{k}\right)>1-\varepsilon$, therefore $k \geq \chi_{\mu}^{\mathrm{ap}}(S, b)$.

It follows that $a \mapsto \chi_{\mu}^{\mathrm{ap}}(S, a)$ is also an invariant of weak equivalence. Next recall the following simple fact.

Proposition 4.4. Let $\Gamma$ be a countable group and $S \subseteq \Gamma$ a set of generators. Then the following are equivalent:

(i) $\chi(\operatorname{Cay}(\Gamma, S))=2$ (i.e., $\operatorname{Cay}(\Gamma, S)$ is bipartite).

(ii) There is a homomorphism $\varphi: \Gamma \rightarrow \mathbb{Z} / 2 \mathbb{Z}$ that sends $S$ to 1 .

(iii) $\left\{s_{1} \ldots s_{2 n}: n \geq 0, s_{i} \in S^{ \pm 1}\right\}$ has index 2 in $\Gamma$.

(iv) For any $s_{1}, \ldots, s_{2 n+1} \in S^{ \pm 1}, n \geq 0$, we have $s_{1} \ldots s_{2 n+1} \neq 1$.

Moreover, a group $\Gamma$ admits a set of generators $S \subseteq \Gamma$ with $\mathrm{Cay}(\Gamma, S)$ bipartite iff $\mathbb{Z} / 2 \mathbb{Z}$ is a factor of $\Gamma$.

We now have:

Proposition 4.5. Let $\Gamma$ be a countable group and $S \subseteq \Gamma$ a set of generators. Let $g=g_{\text {odd }}(\operatorname{Cay}(\Gamma, S))$ be the odd girth of the Cayley graph $\mathrm{Cay}(\Gamma, S)$. Then for any $a \in \operatorname{FR}(\Gamma, X, \mu)$, we have

$$
i_{\mu}(S, a) \leq 1 / 2-1 /(2 g) .
$$

Also if $\Gamma_{0}=\left\{s_{1} \ldots s_{2 n}: n \geq 0, s_{i} \in S^{ \pm 1}\right\}$ and $a \mid \Gamma_{0} \in \mathrm{FR}\left(\Gamma_{0}, X, \mu\right)$ is strongly ergodic, then $i_{\mu}(S, a)<1 / 2$.

Proof. We can assume that $g<\infty$, i.e., that the Cayley graph is not bipartite. Let $A \subseteq X$ be an independent Borel set and let $\mu(A)=1 / 2-\varepsilon$. Then for any $s, t \in S^{ \pm 1}$, it is easy to see that $\mu(A \triangle s t \cdot A) \leq 4 \varepsilon$. So, by induction, if $\gamma=s_{1} \ldots s_{2 n}$, where $s_{i} \in$ $S^{ \pm 1}$, then $\mu(\gamma \cdot A \triangle A) \leq 4 n \varepsilon$. If $g=2 n+1$, then for some $s, s_{1}, \ldots, s_{2 n} \in S^{ \pm 1}$, we have that $s=s_{1} \ldots s_{2 n}$, so $\mu(s \cdot A \triangle A) \leq 4 n \varepsilon$, thus $\varepsilon \geq 1 /(4 n+2)=1 /(2 g)$, therefore $\mu(A) \leq 1 / 2-1 /(2 g)$.

In the case $a \mid \Gamma_{0}$ is strongly ergodic but $i_{\mu}(S, a)=1 / 2$, there are independent Borel sets $A_{n}$ with $\mu\left(A_{n}\right) \rightarrow 1 / 2$. Then for any finite $F \subseteq \Gamma_{0}, \varepsilon>0$, and all large enough $n$, we have $\mu\left(\gamma \cdot A_{n} \triangle A_{n}\right)<\varepsilon, \forall \gamma \in F$, i.e., $a \mid \Gamma_{0}$ has non-trivial almost invariant sets, contradicting strong ergodicity. 
Thus in the context of 4.5 , if $\operatorname{Cay}(\Gamma, S)$ is not bipartite (so that $g<\infty$ ) or if $a \mid \Gamma_{0}$ is strongly ergodic, we have that $i_{\mu}(S, a)<1 / 2$ and $\chi_{\mu}^{\text {ap }}(S, a) \geq 3$. Also recall that if $a \mid \Gamma_{0}$ is ergodic, e.g., if $a$ is weak mixing, then $\chi_{\mu}(S, a) \geq 3$ (otherwise there would be an independent set of measure $1 / 2$ ).

Applying 4.5 to $\Gamma=(\mathbb{Z} / 2 \mathbb{Z}) *(\mathbb{Z} / 3 \mathbb{Z})=\left\langle s, t \mid s^{2}=1, t^{3}=1\right\rangle$, with $S=\{s, t\}$, we have $g=3$, and thus $i_{\mu}(S, a) \leq 1 / 3$, for any $a \in \operatorname{FR}(\Gamma, X, \mu)$. But by 2.19 , since $d=3, i_{\mu}(S, a) \geq 1 / 3$. So $i_{\mu}(S, a)=1 / 3$ for any $a \in \operatorname{FR}(\Gamma, X, \mu)$. This also shows that the upper bound in 4.5 cannot, in general, be improved. We will see at the end of Section 6, using also a result of Lyons-Nazarov [35], that for $\Gamma=(\mathbb{Z} / 3 \mathbb{Z}) *(\mathbb{Z} / 3 \mathbb{Z})=\left\langle s, t \mid s^{3}=1, t^{3}=1\right\rangle$ and $S=\{s, t\}$, we also have $i_{\mu}(S, a)=1 / 3$ for any $a \in \operatorname{FR}(\Gamma, X, \mu)$ and the sup is attained. Also $\chi_{\mu}^{\mathrm{ap}}(S, a)=3$.

Theorem 4.6. Let $\Gamma$ be a countable group and $S \subseteq \Gamma$ a set of generators. Then the following are equivalent:

(i) $\mathrm{Cay}(\Gamma, S)$ is bipartite.

(ii) There is an action $a \in \operatorname{FR}(\Gamma, X, \mu)$ with $i_{\mu}(S, a)=1 / 2$.

(iii) There is an ergodic action $a \in \operatorname{FR}(\Gamma, X, \mu)$ with $i_{\mu}(S, a)=1 / 2$.

(iv) There is an action $a \in \operatorname{FR}(\Gamma, X, \mu)$ with $\chi_{\mu}^{\mathrm{ap}}(S, a)=2$.

(v) There is an ergodic action $a \in \operatorname{FR}(\Gamma, X, \mu)$ with $\chi_{\mu}^{\mathrm{ap}}(S, a)=2$.

(vi) There is an action $a \in \operatorname{FR}(\Gamma, X, \mu)$ with $\chi_{\mu}(S, a)=2$.

(vii) There is an ergodic action $a \in \mathrm{FR}(\Gamma, X, \mu)$ with $\chi_{\mu}(S, a)=2$.

Proof. (ii) $\Rightarrow$ (i) follows from 4.5. Clearly (vii) $\Rightarrow$ (v) $\Rightarrow$ (iii), (vii) $\Rightarrow$ (vi), (v) $\Rightarrow$ (iv), (iii) $\Rightarrow$ (ii) and (iv) $\Rightarrow$ (ii).

Conversely, assume (i) in order to prove (vii). Let $\varphi: \Gamma \rightarrow \mathbb{Z} / 2 \mathbb{Z}$ be a homomorphism that sends $S$ to 1 . Let $a_{0} \in \operatorname{FR}(\Gamma, X, \mu)$ be weakly mixing, and let $a_{1}$ be the action of $\Gamma$ on $\mathbb{Z} / 2 \mathbb{Z}=\{0,1\}$ given by $\gamma \cdot i=\varphi(\gamma)+i$. If $v(\{0\})=v(\{1\})=1 / 2$, then the action of $\Gamma$ on $\mathbb{Z} / 2 \mathbb{Z}$ is measure preserving and ergodic.

Let now $a \in \operatorname{FR}(\Gamma, X \times\{0,1\}, \mu \times v)$ be the product action $a_{0} \times a_{1}$, i.e.,

$$
\gamma \cdot(x, i)=(\gamma \cdot x, \varphi(\gamma)+i) .
$$

Clearly, $X \times\{0\}, X \times\{1\}$ gives a measurable 2-coloring of the graph $G(S, a)$, so $\chi_{\mu}(S, a)=2$. Finally, since $a_{0}$ is free and weakly mixing, the action $a$ is free and ergodic.

(B) Consider now the case of $(\Gamma, S)$, where $S$ is a finite set of generators of $\Gamma$ and $\Gamma$ is amenable. Then if $a, b \in \operatorname{FR}(\Gamma, X, \mu)$ are ergodic, by [23], 13.2, $a \sim b$ and so $i_{\mu}(S, a)=i_{\mu}(S, b)$. Thus $i_{\mu}(S, a)$ is constant for all free, ergodic $a$. Using the ergodic decomposition, this is true for all $a \in \operatorname{FR}(\Gamma, X, \mu)$. We denote by

$$
i_{\mu}(\Gamma, S)
$$

this constant value. 
Similarly, $\chi_{\mu}^{\mathrm{ap}}(S, a)$ is constant for all $a \in \mathrm{FR}(\Gamma, X, \mu)$ and we denote this constant value by

$$
\chi_{\mu}^{\mathrm{ap}}(\Gamma, S)
$$

We now have:

Theorem 4.7. Let $\Gamma$ be a countable, amenable group and $S \subseteq \Gamma$ a finite set of generators. Then

$$
\chi_{\mu}^{\mathrm{ap}}(\Gamma, S)=\chi(\operatorname{Cay}(\Gamma, S)) .
$$

Proof. This follows from Theorem 3.8.

Theorem 4.8. Let $\Gamma$ be a countable, amenable group and $S \subseteq \Gamma$ a finite set of generators. Then:

(i) If $\operatorname{Cay}(\Gamma, S)$ is bipartite, then $i_{\mu}(\Gamma, S)=1 / 2$.

(ii) If $\operatorname{Cay}(\Gamma, S)$ is not bipartite, then $i_{\mu}(\Gamma, S) \leq 1 / 2-1 /(2 g)<1 / 2$, where $g$ is the odd girth of $\mathrm{Cay}(\Gamma, S)$.

Proof. (i) follows from 3.8, and (ii) from 4.5.

We can now identify $i_{\mu}(\Gamma, S)$ in terms of $\operatorname{Cay}(\Gamma, S)$. Recall that a $F \phi l n e r$ sequence in $\Gamma$ is a sequence $\left(F_{n}\right)$ of finite, non-empty subsets of $\Gamma$ such that $\forall \gamma \in \Gamma$, $\frac{\left|\gamma F_{n} \Delta F_{n}\right|}{\left|F_{n}\right|} \rightarrow 0$. We will use the following result that is a consequence of the quasitiling machinery in Ornstein-Weiss [43] and is explicitly stated in Gromov [14], 1.3, and Lindenstrauss-Weiss [31], Appendix (see also Abért, Jaikin-Zapirain and Nikolov [2], Lemma 18).

Theorem 4.9 (Ornstein-Weiss [43], Gromov [14], Lindenstrauss-Weiss [31]). Let $\Gamma$ be an amenable group and $\left(F_{n}\right)$ a Følner sequence in $\Gamma$. Let $h$ be a positive real-valued function defined on all finite subsets of $\Gamma$ such that $h$ is subadditive (i.e., $h(A \cup B) \leq h(A)+h(B))$ and right-invariant $(h(A \gamma)=h(A), \forall \gamma \in \Gamma)$. Then $\lim _{n \rightarrow \infty} \frac{h\left(\bar{F}_{n}\right)}{\left|F_{n}\right|}$ exists (and is of course independent of $\left(F_{n}\right)$ ).

For each finite $F \subseteq \Gamma$ denote by $i(F, S)$ the independence ratio of the induced subgraph $\operatorname{Cay}(\Gamma, S) \mid F$. Then it is easy to check that $h(F)=i(F, S)|F|$ (i.e., $h(F)$ is the maximal cardinality of an independent subset of $\operatorname{Cay}(\Gamma, S) \mid F)$ is subadditive and right-invariant, thus for each Følner sequence $\left(F_{n}\right)$ the limit

$$
\lim _{n \rightarrow \infty} i\left(F_{n}, S\right)=i(\Gamma, S)
$$

exists and is independent of $\left(F_{n}\right)$. We call it the independence number of Cay $(\Gamma, S)$. We now have: 
Theorem 4.10. Let $\Gamma$ be a countable, amenable group and $S \subseteq \Gamma$ a finite set of generators. Then

$$
i_{\mu}(\Gamma, S)=i(\Gamma, S)
$$

Proof. We will use the following two results, the first of which is a consequence of the quasi-tiling machinery of Ornstein-Weiss [43] (see specifically II.§2, Theorem 5 and its subsequent remark, and also the proof of I.\$2, Theorem 6) and the second is a very weak consequence of the mean ergodic theorem for Følner sequences (see, e.g., Nevo [41], 6.7).

Lemma 4.11. (i) Let $\Gamma$ be a countable, amenable group and $1 \in F_{0} \subseteq F_{1} \subseteq \ldots$ an increasing Folner sequence. Let $a \in \operatorname{FR}(\Gamma, X, \mu)$ and $\varepsilon>0$. Then we can find $n_{1}<\cdots<n_{k}$, so that letting $T_{i}=F_{n_{i}}, 1 \leq i \leq k$, we have the following:

For each $1 \leq i \leq k$, there is $l_{i} \geq 1$, sets $T_{i j} \subseteq T_{i}$, and Borel sets $B_{i j} \subseteq X$, $1 \leq j \leq l_{i}$, such that

(a) the sets $T_{i j} B_{i j}, 1 \leq j \leq l_{i}$, are pairwise disjoint;

(b) the sets $t B_{i j}, t \in T_{i j}$, are pairwise disjoint;

(c) the sets $\bigcup_{j \leq l_{i}} T_{i j} B_{i j}, 1 \leq i \leq k$, are pairwise disjoint;

(d) $\mu\left(\bigcup_{i \leq k} \bigcup_{j \leq l_{i}} T_{i j} B_{i j}\right)>1-\varepsilon$;

(e) $\frac{\left|T_{i j}\right|}{\left|T_{i}\right|}>1-\varepsilon, 1 \leq i \leq k, 1 \leq j \leq l_{i}$.

(ii) Let $\Gamma$ be a countable, amenable group and $a \in \mathrm{FR}(\Gamma, X, \mu)$ an ergodic action. Let $\left(F_{n}\right)$ be a Følner sequence for $\Gamma$, let $\varepsilon>0$ and let $A \subseteq X$ be a Borel set. Then for some $n \in \mathbb{N}$ and $x \in X$ we have

$$
\left|\frac{\left|\left\{f \in F_{n}: f \cdot x \in A\right\}\right|}{\left|F_{n}\right|}-\mu(A)\right|<\varepsilon .
$$

It is clearly enough to show that for any ergodic $a \in \operatorname{FR}(\Gamma, X, \mu), i_{\mu}(S, a)=$ $i(\Gamma, S)$. Fix a Følner sequence $1 \in F_{0} \subseteq F_{1} \subseteq \cdots$, in order to show that $\lim _{n \rightarrow \infty} i\left(F_{n}, S\right)=i_{\mu}(S, a)$. We proceed in two steps.

(A) $\lim _{n \rightarrow \infty} i\left(F_{n}, S\right) \leq i_{\mu}(S, a)$.

Put $\alpha=\lim _{n \rightarrow \infty} i\left(F_{n}, S\right)$. We may of course assume that $\alpha>0$. Fix $\alpha>\varepsilon>0$. By (i) of the lemma, applied to an appropriate subsequence of $\left(F_{n}\right)$ and $\varepsilon^{\prime}<<\varepsilon$, we can find $n_{1}<\cdots<n_{k}$ such that letting $T_{i}=F_{n_{i}}, 1 \leq i \leq k$ and letting $T_{i j}, B_{i j}$ be as in the lemma we have the following:

(i) There are independent sets $A_{i j} \subseteq T_{i j}$ with $\left|\frac{\left|A_{i j}\right|}{\left|T_{i j}\right|}-\alpha\right|<\varepsilon$ and $A_{i j} \subseteq\left\{t \in T_{i j}\right.$ : $\left.\forall s \in S^{ \pm 1}\left(s t \in T_{i j}\right)\right\}$.

(ii) The family of sets $\left\{T_{i j} b\right\}$, where $b \in B_{i j}, 1 \leq i \leq k, 1 \leq j \leq l_{i}$, is pairwise disjoint and $\mu\left(\bigcup_{i \leq k} \bigcup_{j \leq l_{i}} T_{i j} B_{i j}\right)>1-\varepsilon$. 
Let then $A=\bigcup_{i \leq k} \bigcup_{j \leq l_{i}} A_{i j} B_{i j}$. Then $A$ is independent and

$$
\begin{aligned}
\mu(A) & =\sum_{i \leq k} \sum_{j \leq l_{i}}\left|A_{i j}\right| \mu\left(B_{i j}\right) \\
& \geq \sum_{i \leq k} \sum_{j \leq l_{i}}(\alpha-\varepsilon)\left|T_{i j}\right| \mu\left(B_{i j}\right) \\
& =(\alpha-\varepsilon) \sum_{i \leq k} \sum_{j \leq l_{i}}\left|T_{i j}\right| \mu\left(B_{i j}\right) \\
& >(\alpha-\varepsilon)(1-\varepsilon),
\end{aligned}
$$

so $i_{\mu}(S, a)>(\alpha-\varepsilon)(1-\varepsilon)$ and thus, letting $\varepsilon \rightarrow 0, i_{\mu}(S, a) \geq \alpha$.

(B) $\lim _{n \rightarrow \infty} i\left(F_{n}, S\right) \geq i_{\mu}(S, a)$.

Let $A \subseteq X$ be an independent Borel set. Fix $\varepsilon>0$ and, by (ii) of the lemma applied to tail ends of $\left(F_{n}\right)$, we can find $x_{1}, x_{2}, \ldots \in X$ and $n_{1}<n_{2}<\cdots$ such that

$$
\left|\frac{\left|\left\{f \in F_{n_{i}}: f \cdot x_{i} \in A\right\}\right|}{\left|F_{n_{i}}\right|}-\mu(A)\right|<\varepsilon .
$$

Let $A_{i}=\left\{f \in F_{n_{i}}: f \cdot x_{i} \in A\right\}$. Then $A_{i}$ is independent in $\operatorname{Cay}(\Gamma, S) \mid F_{n_{i}}$, so $\frac{\left|A_{i}\right|}{\left|F_{n_{i}}\right|} \leq i\left(F_{n_{i}}, S\right)$, thus $\mu(A)<\frac{\left|A_{i}\right|}{\left|F_{n_{i}}\right|}+\varepsilon \leq i\left(F_{n_{i}}, S\right)+\varepsilon$. Letting $i \rightarrow \infty, \varepsilon \rightarrow 0$, we have $\mu(A) \leq \lim _{i \rightarrow \infty} i\left(F_{n_{i}}, S\right)=\lim _{n \rightarrow \infty} i\left(F_{n}, S\right)$.

Remark 4.12. By a similar argument, one can see that if $\Gamma$ is a countable, amenable group and $m$ is a finitely additive, shift-invariant probability measure on $\Gamma$, then

$$
\sup \{m(A): A \subseteq \Gamma \text { is independent in } \operatorname{Cay}(\Gamma, S)\}=i(S, a) .
$$

In particular, this supremum is independent of the choice of $m$.

(C) When $\Gamma$ is not amenable, $i_{\mu}(S, a)$ and $\chi_{\mu}^{\text {ap }}(S, a)$ might not be constant. For example, we have:

Proposition 4.13. Let $\Gamma$ be a countable group and $S \subseteq \Gamma$ a finite set of generators with $\operatorname{Cay}(\Gamma, S)$ bipartite. Then the following are equivalent:

(i) $\Gamma$ is amenable.

(ii) $i_{\mu}(S, a)$ is constant, for all $a \in \mathrm{FR}(\Gamma, X, \mu)$.

(iii) $\chi_{\mu}^{\mathrm{ap}}(S, a)$ is constant, for all $a \in \operatorname{FR}(\Gamma, X, \mu)$.

Proof. We have seen that (i) $\Rightarrow$ (ii), (iii). Assume now that $\Gamma$ is not amenable. Then for the shift action $s_{\Gamma}$ of $\Gamma$ on $2^{\Gamma}$, with the usual product measure, and $\Gamma_{0}$ as in Proposition 4.5, $a \mid \Gamma_{0}$ is strongly ergodic, so $i_{\mu}\left(S, s_{\Gamma}\right)<1 / 2$ and thus also $\chi_{\mu}^{\mathrm{ap}}\left(S, s_{\Gamma}\right) \geq 3$. On the other hand, by 4.6 there is $a \in \operatorname{FR}(\Gamma, X, \mu)$ with $i_{\mu}(S, a)=$ $1 / 2$ and $\chi_{\mu}^{\text {ap }}(S, a)=2$, giving the failure of (ii) and (iii). 
On the other hand, Abért and Weiss [3] showed that among all $a \in \operatorname{FR}(\Gamma, X, \mu)$, there is a minimum one in the sense of weak containment, namely the shift action $s_{\Gamma}$ of $\Gamma$ on $2^{\Gamma}$ (with the usual product measure), and earlier Hjorth (unpublished) and (independently) Glasner-Thouvenot-Weiss [13] showed that there is a maximum one, denoted by $a_{\Gamma, \infty}$ (see also [23], 10.7). Similarly there is a free, ergodic action which is maximum in the sense of weak containment among all the free, ergodic actions, denoted by $a_{\Gamma, \infty}^{\text {erg }}$ (see [23], 13.1). Then for any free, ergodic action $a$,

$$
s_{\Gamma} \prec a \prec a_{\Gamma, \infty}^{\mathrm{erg}} .
$$

Hence for any finite generating set $S \subseteq \Gamma$, we have for any ergodic $a \in \operatorname{FR}(\Gamma, X, \mu)$,

$$
i_{\mu}\left(S, s_{\Gamma}\right) \leq i_{\mu}(S, a) \leq i_{\mu}\left(S, a_{\Gamma, \infty}^{\mathrm{erg}}\right)
$$

and

$$
\chi_{\mu}^{\mathrm{ap}}\left(S, s_{\Gamma}\right) \geq \chi_{\mu}^{\mathrm{ap}}(S, a) \geq \chi_{\mu}^{\mathrm{ap}}\left(S, a_{\Gamma, \infty}^{\mathrm{erg}}\right) .
$$

Thus, by the ergodic decomposition, for any free action $a$,

$$
i_{\mu}\left(S, s_{\Gamma}\right) \leq i_{\mu}(S, a) \leq i_{\mu}\left(S, a_{\Gamma, \infty}^{\mathrm{erg}}\right),
$$

and, using also the proof of 4.3 ,

$$
\chi_{\mu}^{\mathrm{ap}}\left(S, s_{\Gamma}\right) \geq \chi_{\mu}^{\mathrm{ap}}(S, a) \geq \chi_{\mu}^{\mathrm{ap}}\left(S, a_{\Gamma, \infty}^{\mathrm{erg}}\right) .
$$

Note also that if for $0 \leq \alpha, \beta \leq 1$ with $\alpha+\beta=1$, we consider the convex combination $\alpha a+\beta b$, for any free actions $a, b$ (see [23], $10(\mathbf{F})$ ), then trivially $i_{\mu}(S, \alpha a+\beta b)=\alpha i_{\mu}(S, a)+\beta i_{\mu}(S, b)$, therefore $\left\{i_{\mu}(S, a): a \in \operatorname{FR}(\Gamma, X, \mu)\right\}=$ $\left[i_{\mu}\left(S, s_{\Gamma}\right), i_{\mu}\left(S, a_{\Gamma, \infty}^{\mathrm{erg}}\right)\right]$. For example, if $\mathrm{Cay}(\Gamma, S)$ is bipartite and $\Gamma$ is not amenable, then this last interval is not trivial, so $i_{\mu}(S, a)$ takes continuum many values on $\operatorname{FR}(\Gamma, X, \mu)$ and thus, in particular, there are continuum many weak equivalence classes of free actions. Note also that for all these actions $c=\alpha a+\beta b, \alpha, \beta \neq 0$, the corresponding Koopman representations $\kappa^{c}$ (see $\S 4$, (D) below) are all isomorphic (to $\kappa^{a} \oplus \kappa^{b}$ ). It is not clear however what is the range of $i_{\mu}(S, a)$ on the space of ergodic, free actions.

We next show that for $(\Gamma, S)$ with $\operatorname{Cay}(\Gamma, S)$ bipartite, one can characterize whether $\Gamma$ is amenable, has property $(\mathrm{T})$ or the HAP in terms of the independence and approximate chromatic numbers of its actions. We start with the following characterization of amenability.

Theorem 4.14. Let $\Gamma$ be a countable group and $S \subseteq \Gamma$ a finite set of generators such that $\mathrm{Cay}(\Gamma, S)$ is bipartite. Then the following are equivalent:

(i) $\Gamma$ is amenable.

(ii) $i_{\mu}(S, a)=1 / 2$, for any $a \in \mathrm{FR}(\Gamma, X, \mu)$.

(iii) $\chi_{\mu}^{\mathrm{ap}}(S, a)=2$, for any $a \in \mathrm{FR}(\Gamma, X, \mu)$. 
(iv) $i_{\mu}\left(S, s_{\Gamma}\right)=1 / 2$.

(v) $\chi_{\mu}^{\mathrm{ap}}\left(S, s_{\Gamma}\right)=2$.

In particular, if $\Gamma$ is a finitely generated group having $\mathbb{Z} / 2 \mathbb{Z}$ as a factor, then the following are equivalent:

(a) $\Gamma$ is amenable.

(b) There is a finite generating set $S \subseteq \Gamma$ such that $i_{\mu}\left(S, s_{\Gamma}\right)=1 / 2$.

(c) As in (b) with $\chi_{\mu}^{\mathrm{ap}}\left(S, s_{\Gamma}\right)=2$.

Proof. This follows from 4.13 and its proof.

We next consider property (T) and the HAP.

Theorem 4.15. Let $\Gamma$ be an infinite, countable group and $S \subseteq \Gamma$ a finite set of generators such that $\mathrm{Cay}(\Gamma, S)$ is bipartite. Then the following are equivalent:

(i) $\Gamma$ has property (T).

(ii) $i_{\mu}(S, a)<1 / 2$, for every weakly mixing $a \in \operatorname{FR}(\Gamma, X, \mu)$.

(iii) $\chi_{\mu}^{\mathrm{ap}}(S, a) \geq 3$, for every weakly mixing $a \in \mathrm{FR}(\Gamma, X, \mu)$.

Also the following are equivalent:

(i*) $\Gamma$ does not have the HAP.

(ii*) $i_{\mu}(S, a)<1 / 2$, for every mixing $a \in \operatorname{FR}(\Gamma, X, \mu)$.

(iii*) $\chi_{\mu}^{\mathrm{ap}}(S, a) \geq 3$, for every mixing $a \in \mathrm{FR}(\Gamma, X, \mu)$.

Proof. Suppose first that $\Gamma$ has property (T). If $\Gamma_{0}=\left\{s_{1} s_{2} \ldots s_{2 n}: n \geq 0, s_{i} \in\right.$ $S^{ \pm 1}$, then $\Gamma_{0}$ has index 2 in $\Gamma$ and thus $\Gamma_{0}$ itself has property (T). Moreover, if $a \in \operatorname{FR}(\Gamma, X, \mu)$ is weakly mixing, then $a \mid \Gamma_{0} \in \mathrm{FR}\left(\Gamma_{0}, X, \mu\right)$ is ergodic, so strongly ergodic (see, e.g., [23], 11.2), thus $i_{\mu}(S, a)<1 / 2$ by Proposition 4.5 . So (i) $\Rightarrow$ (ii) $\Rightarrow$ (iii).

Assume now that $\Gamma$ does not have property (T). By 4.6 there is $b \in \operatorname{FR}(\Gamma, X, \mu)$ with $i_{\mu}(S, b)=1 / 2$ and $\chi_{\mu}^{\mathrm{ap}}(S, b)=2$. By a result of Kerr-Pichot [27] (see also [23], 12.9), there is a weakly mixing $a \in \mathrm{FR}(\Gamma, X, \mu)$ with $b \prec a$, so $i_{\mu}(S, b) \leq i_{\mu}(S, a)$, thus $i_{\mu}(S, a)=1 / 2$, and $\chi_{\mu}^{\mathrm{ap}}(S, b) \geq \chi_{\mu}^{\mathrm{ap}}(S, a)$, therefore $\chi_{\mu}^{\mathrm{ap}}(S, a)=2$.

If now $\Gamma$ does not have the HAP and $a \in \operatorname{FR}(\Gamma, X, \mu)$ is mixing, then $\Gamma_{0}$ as above does not have the HAP, and $a \mid \Gamma_{0} \in \mathrm{FR}\left(\Gamma_{0}, X, \mu\right)$ is mixing, so (see, e.g., [23], 11.1) it is strongly ergodic, thus $i_{\mu}(S, a)<1 / 2$ as before. So $\left(\mathrm{i}^{*}\right) \Rightarrow$ (ii*) $\Rightarrow$ (iii*).

Conversely, if $\Gamma$ has the HAP, then we can repeat the argument above (for the case that $\Gamma$ does not have property $(\mathrm{T})$ ) using the result of Hjorth [16] (see also [23], 12.11) to replace in this argument weakly mixing by mixing.

(D) For any unitary representation $\pi: \Gamma \rightarrow U(H)$ of a countable group $\Gamma$ on a Hilbert space $H$, and a finite set of generators $S \subseteq \Gamma$, one defines the averaging 
operator $T_{S, \pi}$ by

$$
T_{S, \pi}(f)=\frac{1}{\left|S^{ \pm 1}\right|} \sum_{s \in S^{ \pm 1}} \pi(s)(f)
$$

Clearly $T_{S, \pi}$ is a self-adjoint operator and $\left\|T_{s, \pi}\right\| \leq 1$. It is easy to check that if $\pi$, $\rho$ are unitary representations and $\pi$ is weakly contained in $\rho$ (see, e.g, Bekka-de la Harpe-Valette [5], Appendix F), which is denoted by $\pi \prec \rho$, then $\left\|T_{s, \pi}\right\| \leq\left\|T_{s, \rho}\right\|$, i.e.,

$$
\pi \prec \rho \Longrightarrow\left\|T_{s, \pi}\right\| \leq\left\|T_{s, \rho}\right\| .
$$

When $\Gamma$ is amenable, Kesten [28] showed that $\left\|T_{S, \lambda_{\Gamma}}\right\|=1$, where $\lambda_{\Gamma}$ is the (left) regular representation of $\Gamma$.

For each $a \in \operatorname{FR}(\Gamma, X, \mu)$, consider the corresponding Koopman unitary representation $\kappa^{a}$ on $L^{2}(X, \mu)$ and its restriction $\kappa_{0}^{a}$ on $L_{0}^{2}(X, \mu)=\left\{f \in L^{2}(X, \mu)\right.$ : $\left.\int f d \mu=0\right\}=\mathbb{C}^{\perp}$ (where $\mathbb{C}$ is identified with the subspace of constant functions in $L^{2}(X, \mu)$ ). Then for a finite generating set $S \subseteq \Gamma$, let

$$
T_{S, a}=T_{S, \kappa_{0}^{a}}
$$

There is a well-known connection between norms of averaging operators and independence ratios in the case of finite graphs, due to Hoffman [18] (see, e.g., Davidoff-Sarnak-Valette [9], 1.5.3), and a version of this carries over to our context.

Proposition 4.16. Let $\Gamma$ be a countable group and $S \subseteq \Gamma$ a finite set of generators. Let $a \in \operatorname{FR}(\Gamma, X, \mu)$ and let $T_{S, a}$ the corresponding averaging operator. If $v=$ $\left\|T_{S, a}\right\|$, then

$$
i_{\mu}(S, a) \leq \frac{v}{1+v}
$$

and thus

$$
\chi_{\mu}^{\mathrm{ap}}(S, a) \geq \frac{1+v}{v} .
$$

Proof. Let $A \subseteq X$ be independent and let $T=T_{S, a}, f=\chi_{A}-\mu(A)$. Then $f \in L_{0}^{2}(X, \mu)$ and

$$
\|f\|^{2}=\mu(A)(1-\mu(A))
$$


Also,

$$
\begin{aligned}
\langle T(f), f\rangle & =\int T(f)(x) f(x) d \mu(x) \\
& =\frac{1}{\left|S^{ \pm 1}\right|} \sum_{s \in S^{ \pm 1}} \int f(s \cdot x) f(x) d \mu(x) \\
& =\frac{1}{\left|S^{ \pm 1}\right|} \sum_{s \in S^{ \pm 1}} \int\left(\chi_{s^{-1} \cdot A}(x)-\mu(A)\right)\left(\chi_{A}(x)-\mu(A)\right) d \mu(x) \\
& =\frac{1}{\left|S^{ \pm 1}\right|} \sum_{s \in S^{ \pm 1}} \int\left(-\mu(A)\left(\chi_{A}(x)+\chi_{s^{-1} \cdot A}(x)\right)+\mu(A)^{2}\right) d \mu(x) \\
& =\frac{1}{\left|S^{ \pm 1}\right|} \sum_{s \in S^{ \pm 1}}\left(-2 \mu(A)^{2}+\mu(A)^{2}\right) \\
& =-\mu(A)^{2} .
\end{aligned}
$$

Since $|\langle T(f), f\rangle| \leq\|T\| \cdot\|f\|^{2}$, letting $\alpha=\mu(A)$, we have

$$
\alpha^{2} \leq v \cdot \alpha(1-\alpha)
$$

so

$$
\alpha \leq \frac{v}{1+v}
$$

Since for $a, b \in \operatorname{FR}(\Gamma, X, \mu)$,

$$
a \prec b \Longrightarrow \kappa_{0}^{a} \prec \kappa_{0}^{b}
$$

(see [23], 10.5), it follows that

$$
a \prec b \Longrightarrow\left\|T_{s, a}\right\| \leq\left\|T_{s, b}\right\|
$$

(in fact it is not hard to see that $a \mapsto\left\|T_{s, a}\right\|$ is lower semicontinuous), thus, since $s_{\Gamma} \prec a, \forall a \in \operatorname{FR}(\Gamma, X, \mu),\left\|T_{S, s_{\Gamma}}\right\|$ is minimum among all such $\left\|T_{s, a}\right\|$. Now it is well known (see, e.g., [5], E.4.5) that $\kappa_{0}^{s_{\Gamma}} \sim \lambda_{\Gamma}$, thus $\left\|T_{S, s_{\Gamma}}\right\|=\left\|T_{S, \lambda_{\Gamma}}\right\|$.

Suppose now that $S=\left\{\gamma_{1}, \ldots, \gamma_{m}\right\}$. Kesten [28] has shown that

$$
\left\|T_{S, \lambda_{\Gamma}}\right\| \geq \frac{\sqrt{2 m-1}}{m}
$$

and if $S$ is a free set of generators, so that $\Gamma=\mathbb{F}_{m}$, then

$$
\left\|T_{S, \lambda_{\Gamma}}\right\|=\frac{\sqrt{2 m-1}}{m} .
$$


Also, if $S=\left\{\gamma_{1}, \ldots, \gamma_{m}, \delta_{1}, \ldots, \delta_{n}\right\}$, where $\gamma_{1}, \ldots, \gamma_{m}$ are free and $\delta_{1}, \ldots, \delta_{n}$ are free satisfying $\delta_{i}^{2}=1, i=1, \ldots, n$, so that $\operatorname{Cay}(\Gamma, S)$ is still acyclic and $\Gamma=\mathbb{F}_{m} * \mathbb{Z} / 2 \mathbb{Z} * \cdots * \mathbb{Z} / 2 \mathbb{Z}$ ( $n$ times), then again

$$
\left\|T_{S, \lambda_{\Gamma}}\right\|=\frac{2 \sqrt{(2 m+n)-1}}{2 m+n} .
$$

We then have:

Theorem 4.17. Let $\Gamma=\mathbb{F}_{m}$ be the free group with a free set $S$ of $m$ generators, and $s_{\Gamma}$ its shift action on $2^{\Gamma}$, with the product measure $\mu$. Then

$$
\frac{1}{2 m} \leq i_{\mu}\left(S, s_{\Gamma}\right) \leq \frac{\sqrt{2 m-1}}{m+\sqrt{2 m-1}}
$$

and

$$
2 m \geq \chi_{\mu}^{\mathrm{ap}}\left(S, s_{\Gamma}\right) \geq \frac{m+\sqrt{2 m-1}}{\sqrt{2 m-1}}
$$

Moreover,

$$
2 m+1 \geq \chi_{B}\left(S, s_{\Gamma}\right)
$$

(where we view in the last inequality $s_{\Gamma}$ as the shift action restricted to its free part).

Proof. The first part follows from Theorem 2.19, Propositions 2.13, 4.16, and the preceding paragraphs. The last part follows from [24], 4.6.

This, in particular, gives examples of m.p., ergodic Borel graphs of bounded degree which are acyclic but the approximate chromatic numbers and thus the measurable and Borel chromatic numbers are finite but tend towards $\infty$.

An analogous result to Theorem 4.17 holds when $\Gamma=\mathbb{F}_{m} * \mathbb{Z} / 2 \mathbb{Z} * \cdots * \mathbb{Z} / 2 \mathbb{Z}$ ( $n$ times).

It is mentioned in Lyons-Nazarov [35] that from results of Bollobás and FriezeLuczak concerning random regular graphs, it follows that, for large enough $m$, one has for $\Gamma=\mathbb{F}_{m}$, and $S$ a free set of generators, $i_{\mu}\left(S, s_{\Gamma}\right) \leq \frac{\log 2 m}{m}$ and so $\chi_{\mu}^{\mathrm{ap}}\left(S, s_{\Gamma}\right) \geq$ $\frac{m}{\log 2 m}$. For references, see Section 5 of [35].

We do not know what are the exact values of $i_{\mu}\left(S, s_{\Gamma}\right), \chi_{\mu}^{\mathrm{ap}}\left(S, s_{\Gamma}\right)$ for $\Gamma=\mathbb{F}_{m}$ and $S$ a free set of generators (similarly for $\chi_{B}\left(S, s_{\Gamma}\right), \chi_{\mu}\left(S, s_{\Gamma}\right)$ ).

Concerning Borel chromatic numbers of shifts, denote below by $s_{\Gamma}$ the restriction of the shift action of $\Gamma$ on $2^{\Gamma}$ to its free part, and recall that for a generating set $S \subseteq \Gamma$, $\chi_{B}\left(S, s_{\Gamma}\right)$ denotes the Borel chromatic number associated with $s_{\Gamma}$. If $\Gamma=\mathbb{Z}^{m}$, with $S$ the usual set of $m$ generators, then Gao-Jackson [12] showed that $\chi_{B}\left(S, s_{\Gamma}\right) \in\{3,4\}$, while of course $\chi\left(G\left(S, s_{\Gamma}\right)\right)=\chi(\operatorname{Cay}(\Gamma, S))=2$. For a generalization of the $m=2$ case, see 5.15 below. Gao-Jackson-Miller (unpublished) and recently Adam Timar (private communication) have shown that in this setting $\chi_{\mu}\left(S, s_{\Gamma}\right)=3$. 
Is it true that there is a function $f: \mathbb{N} \rightarrow \mathbb{N}$ such that if $\Gamma$ is amenable and $S$ is any finite generating set, then $\chi_{B}\left(S, s_{\Gamma}\right) \leq f(\chi(\operatorname{Cay}(\Gamma, S)))$ ? (Note that, by 4.7, this is true for $\chi_{\mu}^{\text {ap }}$ with $f=$ id.) We do not know a counterexample even for $f(n)=n+1$.

On the other hand, if $\Gamma$ is finitely generated with $\mathbb{F}_{2} \leq \Gamma$ and $\mathbb{Z} / 2 \mathbb{Z}$ is a factor of $\Gamma$, then for each $\varepsilon>0$, there is a finite generating set $S \subseteq \Gamma$ with $\chi(\operatorname{Cay}(\Gamma, S))=2$, but $i_{\mu}\left(S, s_{\Gamma}\right)<\varepsilon$ and so $\chi_{B}\left(S, s_{\Gamma}\right) \geq \chi_{\mu}\left(S, s_{\Gamma}\right) \geq \chi_{\mu}^{\text {ap }}\left(S, s_{\Gamma}\right)>1 / \varepsilon$. Indeed, choose $m$ large enough so that $\frac{\sqrt{2 m-1}}{m+\sqrt{2 m-1}}<\varepsilon$ and let $\varphi: \Gamma \rightarrow \mathbb{Z} / 2 \mathbb{Z}$ be a surjective homomorphism. Then $\Gamma_{0}=\operatorname{ker}(\varphi)$ contains a free subgroup $\Delta=\left\langle a_{1}, \ldots, a_{m}\right\rangle$ with $m$ free generators. Let $S_{0} \supset\left\{a_{1}, \ldots, a_{m}\right\}$ be a finite set of generators for $\Gamma_{0}$ and let $a \notin \Gamma_{0}$. Put $S=\{a\} \cup a S_{0}$. Clearly $S$ generates $\Gamma$ and there are no odd cycles in $\operatorname{Cay}(\Gamma, S)$, so $\chi(\operatorname{Cay}(\Gamma, S))=2$. However, if $A$ is an independent set in the graph associated with $s_{\Gamma}$, then it is independent for the graph associated with the action $s_{\Gamma} \mid \Delta$ and the set of generators $S_{\Delta}=\left\{a_{1}, \ldots, a_{m}\right\}$. One can again see that $\kappa_{0}^{s \Gamma \mid \Delta} \sim \lambda_{\Delta}$, so

$$
i_{\mu}\left(S, s_{\Gamma}\right) \leq i_{\mu}\left(S_{\Delta}, s_{\Gamma} \mid \Delta\right) \leq \frac{\sqrt{2 m-1}}{m+\sqrt{2 m-1}}<\varepsilon .
$$

We should finally mention that although we have examples where

$$
\chi_{\mu}^{\mathrm{ap}}(S, a)<\chi_{\mu}(S, a)
$$

(see 2.8 or take any weakly mixing action $a$, for which therefore $\chi_{\mu}(S, a) \geq 3$, with $\left.\chi_{\mu}^{\mathrm{ap}}(S, a)=2\right)$, we do not know any examples for which $\chi_{\mu}^{\mathrm{ap}}(S, a)+1<\chi_{\mu}(S, a)$. R. Lyons (private communication) also asked if there are examples of strongly ergodic (also known as $E_{0}$-ergodic) actions $a$ with $\chi_{\mu}^{\text {ap }}(S, a)<\chi_{\mu}(S, a)$.

Remark 4.18. Let $S_{m}=\left\{\gamma_{1}, \ldots, \gamma_{m}\right\}$ be a free set of generators for $\mathbb{F}_{m}, m \geq 1$. Denote by $i_{m}=i_{\mu}\left(S_{m}, s_{\mathbb{F}_{m}}\right)$ the independence number of the shift action of $\mathbb{F}_{m}$, i.e., the minimum independence number of a free, measure-preserving action of $\mathbb{F}_{m}$. Here and below we abuse notation by using the same subscript $\mu$ for the associated measure of any action discussed below. From the preceding theorem we have that $i_{m} \rightarrow 0$ as $m \rightarrow \infty$. Let us also note the following:

(i) $\forall m\left(i_{m+1} \leq i_{m}\right)$.

To see this, consider the shift action $s_{\mathbb{F}_{m+1}}$ and its restriction $a=s_{\mathbb{F}_{m+1}} \mid \mathbb{F}_{m}$, where we view $\mathbb{F}_{m}$ as the subgroup generated by $\gamma_{1}, \ldots, \gamma_{m}$. Clearly if $A \subseteq 2^{\mathbb{F}_{m+1}}$ is independent for $s_{\mathbb{F}_{m+1}}$, it is also independent for $a$, thus

$$
i_{m+1} \leq i_{\mu}\left(S_{m}, a\right) .
$$

Moreover $a \cong\left(s_{\mathbb{F}_{m}}\right)^{\mathbb{N}}$, where $\left(s_{\mathbb{F}_{m}}\right)^{\mathbb{N}}$ is the product of countably many copies of $s_{\mathbb{F}_{m}}$, i.e., it is the action of $\mathbb{F}_{m}$ on $\left(2^{\mathbb{F} m}\right)^{\mathbb{N}}$, given by

$$
\gamma \cdot\left(p_{n}\right)=\left(\gamma \cdot p_{n}\right), \forall \gamma \in \mathbb{F}_{m} .
$$


Now $\left(s_{\mathbb{F}_{m}}\right)^{\mathbb{N}} \cong s_{\mathbb{F}_{m}}^{*}$, where $s_{\mathbb{F}_{m}}^{*}$ is the shift action of $\mathbb{F}_{m}$ on $\left(2^{\mathbb{N}}\right)^{\mathbb{F}_{m}}$. The isomorphism is given by the map

$$
\left(p_{0}, p_{1}, \ldots\right) \in\left(2^{\mathbb{F}_{m}}\right)^{\mathbb{N}} \mapsto p \in\left(2^{\mathbb{N}}\right)^{\mathbb{F}_{m}},
$$

where $p(\gamma)=\left(p_{0}(\gamma), p_{1}(\gamma), \ldots\right), \forall \gamma \in \mathbb{F}_{m}$. (Here all these product spaces have the product measures arising from the $(1 / 2,1 / 2)$-measure on $2=\{0,1\}$.) Now Bowen [7] has shown that $s_{\mathbb{F}_{m}} \sim s_{\mathbb{F}_{m}}^{*}$, thus

$$
i_{m+1} \leq i_{\mu}\left(S_{m}, a\right)=i_{\mu}\left(S_{m}, s_{\mathbb{F}_{m}}^{*}\right)=i_{\mu}\left(S_{m}, s_{\mathbb{F}_{m}}\right)=i_{m} .
$$

It follows that for infinitely many $m, i_{m+1}<i_{m}$. For such $m \geq 2$, one can see that there are at least three distinct values of $i_{\mu}\left(S_{m+1}, a\right)$, as $a$ varies over ergodic actions in $\operatorname{FR}\left(\mathbb{F}_{m+1}, X, \mu\right)$. This is a small initial step towards trying to understand the possible values of the independence number of free, ergodic actions of a free group (see the penultimate paragraph preceding 4.14). Recalling that the maximum value of $i_{\mu}\left(S_{m+1}, a\right)$, for ergodic $a \in \operatorname{FR}\left(\mathbb{F}_{m+1}, X, \mu\right)$, is equal to $1 / 2$, this will follow from the following fact:

(ii) Let $m \geq 2$. Then there is a free, ergodic action $b$ of $\mathbb{F}_{m+1}$ such that

$$
i_{m} \leq i_{\mu}\left(S_{m+1}, b\right)<1 / 2 .
$$

To see this, let $\varphi: \mathbb{F}_{m+1} \rightarrow \mathbb{F}_{m}$ be the homomorphism defined by $\varphi\left(\gamma_{i}\right)=\gamma_{i}$, if $i \leq m, \varphi\left(\gamma_{m+1}\right)=\gamma_{m}$. Let $d=s_{\mathbb{F}_{m}}$ and let $c$ be the lift of $d$ to $\mathbb{F}_{m+1}$ via $\varphi$ :

$$
\gamma^{c}(x)=\varphi(\gamma)^{d}(x) \text {. }
$$

Clearly $c \mid \mathbb{F}_{m}=d$ and $i_{\mu}\left(S_{m+1}, c\right)=i_{\mu}\left(S_{m}, d\right)$. Put

$$
b=c \times s_{\mathbb{F}_{m+1}} .
$$

Then $b \in \mathrm{FR}\left(\mathbb{F}_{m+1}, X, \mu\right)$ and $b$ is ergodic. We will show that $b$ is strongly ergodic thus, by $4.5, i_{\mu}\left(S_{m+1}, b\right)<1 / 2$. But also

$$
i_{\mu}\left(S_{m+1}, b\right) \geq i_{\mu}\left(S_{m+1}, c\right)=i_{\mu}\left(S_{m}, d\right)=i_{m} .
$$

If $b$ is not strongly ergodic, towards a contradiction, there exist almost invariant sets for $b \mid \Gamma_{0}$, where $\Gamma_{0} \leq \mathbb{F}_{m+1}$ is the group of words of even length in $\left\{\gamma_{1}, \ldots, \gamma_{m+1}\right\}$, and thus there exist almost invariant sets for $b \mid \Gamma_{0}^{\prime}$, where $\Gamma_{0}^{\prime} \leq \mathbb{F}_{m}$ is the analogous group of words of even length in $\left\{\gamma_{1}, \ldots, \gamma_{m}\right\}$. But

$$
\begin{aligned}
b \mid \mathbb{F}_{m} & =\left(c \times s_{\mathbb{F}_{m+1}}\right) \mid \mathbb{F}_{m} \\
& =\left(c \mid \mathbb{F}_{m}\right) \times\left(s_{\mathbb{F}_{m+1}} \mid \mathbb{F}_{m}\right) \\
& \simeq d \times\left(s_{\mathbb{F}_{m}}\right)^{\mathbb{N}} \\
& \sim s_{\mathbb{F}_{m}} \times s_{\mathbb{F}_{m}} \\
& \sim s_{\mathbb{F}_{m}},
\end{aligned}
$$

so $b\left|\Gamma_{0}^{\prime} \sim s_{\mathbb{F}_{m}}\right| \Gamma_{0}^{\prime}$, which is strongly ergodic (see, e.g., [17], A4.1), a contradiction. 
(E) We will next see some connections with finite graphs.

Let $\Gamma$ be a countable group and fix a sequence $F_{1} \subseteq F_{2} \subseteq \cdots \subseteq \Gamma$ of finite, non-empty subsets of $\Gamma$ with $\bigcup_{n} F_{n}=\Gamma$. Consider the space $2^{\Gamma}$ with the product topology. If $p \in 2^{F_{n}}$, let

$$
\mathcal{N}_{p}=\left\{f \in 2^{\Gamma}: f \mid F_{n}=p\right\} .
$$

Then $\left\{\mathcal{N}_{p}\right\}_{n \geq 1, p \in 2 F_{n}}$ is a clopen basis for the topology of $2^{\Gamma}$. Let now $S \subseteq \Gamma$ be a set of generators for $\Gamma$. Consider the finite graph with loops $G_{S, n}=\left(2^{F_{n}}, E_{S, n}\right)$, where

$$
p E_{S, n} q \Longleftrightarrow \exists s \in S\left(s^{ \pm 1} \cdot \mathcal{N}_{p} \cap \mathcal{N}_{q} \neq \emptyset\right) .
$$

Here $\gamma \cdot f\left(\gamma \in \Gamma, f \in 2^{\Gamma}\right)$ refers to the shift action of $\Gamma$ on $2^{\Gamma}$. Thus, there is a loop from $p$ to $p$ iff $\exists s \in S\left(s \cdot \mathcal{N}_{p} \cap \mathcal{N}_{p} \neq \emptyset\right)$. We may view each $G_{S, n}$ as a finite approximation of the graph associated with the shift action of $\Gamma$. Below recall that for a finite graph with loops $G=(X, E)$ its independence ratio $i(G)$ is defined as the ratio of the largest size of an independent set divided by $|X|$. When we have a graph with loops we define an independent set to be one for which there are no edges between two (not necessarily distinct) elements of $A$ (thus, $A$ cannot contain any vertex incident with a loop).

Theorem 4.19. For the graphs $G_{S, n}$ as above, $i\left(G_{S, n}\right) \leq i\left(G_{S, n+1}\right), \forall n \geq 1$, and

$$
\lim _{n \rightarrow \infty} i\left(G_{S, n}\right)=i_{\mu}\left(S, s_{\Gamma}\right),
$$

where $\mu$ is the usual product measure on $2^{\Gamma}$.

Proof. Let $A \subseteq 2^{F_{n}}$ be an independent set for $G_{S, n}$, i.e., for $p, q \in A$ (not necessarily distinct), $s \cdot \mathcal{N}_{p} \cap \mathcal{N}_{q}=\emptyset, \forall s \in S^{ \pm 1}$. This is the same thing as saying that

$$
s \cdot\left(\bigcup_{p \in A} \mathcal{N}_{p}\right) \cap \bigcup_{p \in A} \mathcal{N}_{p}=\emptyset, \forall s \in S^{ \pm 1} .
$$

Let $A^{\prime} \subseteq 2^{F_{n+1}}$ be defined by

$$
q \in A^{\prime} \Longleftrightarrow q \mid F_{n} \in A .
$$

Then $\frac{|A|}{\mid 2^{F_{n} \mid}}=\frac{\left|A^{\prime}\right|}{\left|2^{F_{n}+1}\right|}$ and for $p \in A, \mathcal{N}_{p}=\bigcup_{q \in A^{\prime}, q \mid F_{n}=p} \mathcal{N}_{q}$, so $\bigcup_{p \in A} \mathcal{N}_{p}=$ $\bigcup_{p \in A} \bigcup_{q \in A^{\prime}, q \mid F_{n}=p} \mathcal{N}_{q}=\bigcup_{q \in A^{\prime}} \mathcal{N}_{q}$, so $s \cdot\left(\bigcup_{q \in A^{\prime}} \mathcal{N}_{q}\right) \cap \bigcup_{q \in A^{\prime}} \mathcal{N}_{q}=\emptyset, \forall s \in$ $S^{ \pm 1}$, i.e., $A^{\prime}$ is independent for $G_{S, n+1}$. Thus $i\left(G_{S, n}\right) \leq i\left(G_{S, n+1}\right)$.

Also if $A$ is independent for $G_{S, n}$ and $\hat{A}=\bigcup_{p \in A} \mathcal{N}_{p}$, then $\widehat{A}$ is independent for $G\left(S, s_{\Gamma}\right)$ and $\frac{|A|}{\left|2^{F}\right|}=\mu(\widehat{A})$, thus $i\left(G_{S, n}\right) \leq i_{\mu}\left(S, s_{\Gamma}\right)$.

Assume now that $\alpha<i_{\mu}\left(S, s_{\Gamma}\right)$ and let $B \subseteq 2^{\Gamma}$ be an independent Borel set for $G\left(S, s_{\Gamma}\right)$ with $\mu(B)>\alpha$. Let $\varepsilon>0$ and let $K \subseteq 2^{\Gamma}$ be compact with $K \subseteq B$, 
$\mu(B \backslash K)<\varepsilon$. Then $K$ is also independent, so $s \cdot K \cap K=\emptyset, \forall s \in S^{ \pm 1}$. Since the shift action is continuous, there is an open set $U \supseteq K$ with $\mu(U \backslash K)<\varepsilon$ such that $s \cdot U \cap U=\emptyset, \forall s \in S^{ \pm 1}$, i.e., $U$ is also independent. By compactness, let now $n$ be large enough and $A \subseteq 2^{F_{n}}$ be such that $K \subseteq \widehat{A} \subseteq U$ (where, as before, $\hat{A}=$ $\left.\bigcup_{p \in A} \mathcal{N}_{p}\right)$. Thus $A$ is independent in $G_{S, n}$ and so $\alpha-\varepsilon \leq \mu(\widehat{A})=\frac{|A|}{\mid 2^{F_{n} \mid}} \leq i\left(G_{S, n}\right)$. Letting $\varepsilon \rightarrow 0$ we have that

$$
\alpha \leq \lim _{n \rightarrow \infty} i\left(G_{S, n}\right)
$$

so

$$
\lim _{n \rightarrow \infty} i\left(G_{S, n}\right)=i_{\mu}\left(S, s_{\Gamma}\right) .
$$

For the next result, if $G=(X, E)$ is a graph with loops, by a cycle we understand a sequence of distinct elements $x_{1}, \ldots, x_{n}$ of $X$ and distinct edges $e_{1}, \ldots, e_{m}$ such that each $e_{i}$ is either an edge connecting some $x_{j}, x_{j+1}, 1 \leq k<n$, or $x_{n}, x_{0}$, or else a loop incident with some $x_{j}$, and moreover there is an edge $e_{i}$ from each $x_{j}$ to $x_{j+1}$, $1 \leq j<n$, and from $x_{n}$ to $x_{0}$. The length of this cycle is the number $m$ of edges. For example

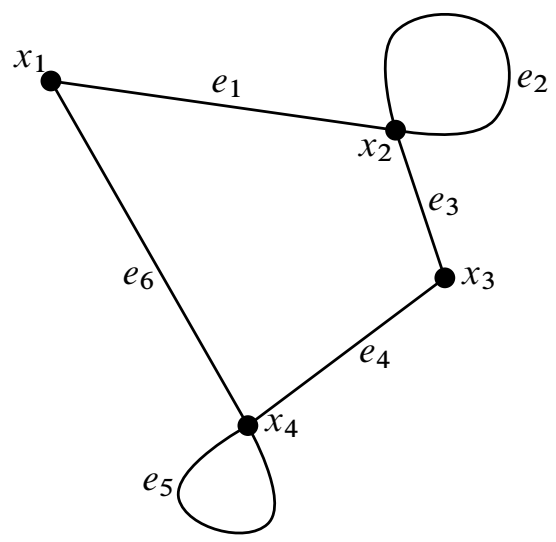

is a cycle of length 6 .

Theorem 4.20. If $\operatorname{Cay}(\Gamma, S)$ is bipartite, then if

$$
X_{S, k, n}=\left\{p \in 2^{F_{n}}: p \text { belongs to an odd cycle of length } \leq k \text { in } G_{S, n}\right\},
$$

we have $\frac{\left|X_{S, k, n}\right|}{\left|2^{F_{n}}\right|} \rightarrow 0$.

Proof. If $\hat{X}_{S, k, n}=\bigcup\left\{\mathcal{N}_{p}: p \in X_{S, k, n}\right\}$, we will show that

$$
\mu\left(\hat{X}_{S, k, n}\right)=\frac{\left|X_{S, k, n}\right|}{\left|2^{F_{n}}\right|} \rightarrow 0 .
$$


Let $X \subseteq 2^{\Gamma}$ be the free part of the shift action of $\Gamma$ on $2^{\Gamma}$, so that $\mu(X)=1$. It is enough to show that

$$
\bigcap_{n \geq 1} \bigcup_{m \geq n} \hat{X}_{S, k, m} \subseteq 2^{\Gamma} \backslash X .
$$

(Then $\lim _{n \rightarrow \infty} \mu\left(\bigcup_{m \geq n} \hat{X}_{S, k, m}\right)=0$, so $\lim _{n \rightarrow \infty} \mu\left(\hat{X}_{S, k, n}\right)=0$.)

Fix $x \in \bigcap_{n} \bigcup_{m \geq n} \hat{X}_{S, k, m}$. Then $x \in \hat{X}_{S, k, n_{i}}$, where $1<n_{1}<n_{2}<\cdots$, so $x \in \mathcal{N}_{p_{i}}$, where $p_{i} \in X_{S, k, n_{i}}$. Then $p_{i}$ belongs to some odd cycle of length $\leq k$, so, by going to a subsequence of $\left(n_{i}\right)$, we may assume that every $p_{i}$ belongs to a $(2 l+1)$ cycle for some $l$ with $2 l+1 \leq k$. Then there are $p_{i}^{0}=p_{i}, p_{i}^{1}, \ldots, p_{i}^{2 l}$ in $X_{S, k, n_{i}}$ and $s_{i}^{0}, s_{i}^{1}, \ldots, s_{i}^{2 l}$ in $S^{ \pm 1}$ with

$$
\begin{aligned}
& s_{i}^{0} \cdot \mathcal{N}_{p_{i}^{0}} \cap \mathcal{N}_{p_{i}^{1}} \neq \emptyset, \\
& s_{i}^{1} \cdot \mathcal{N}_{p_{i}^{1}} \cap \mathcal{N}_{p_{i}^{2}} \neq \emptyset, \\
& \vdots \\
& s_{i}^{2 l-1} \cdot \mathcal{N}_{p_{i}^{2 l-1}} \cap \mathcal{N}_{p_{i}^{2 l}} \neq \emptyset, \\
& s_{i}^{2 l} \cdot \mathcal{N}_{p_{i}^{2 l}} \cap \mathcal{N}_{p_{i}^{0}} \neq \emptyset .
\end{aligned}
$$

By again passing to a subsequence of $\left(n_{i}\right)$, we may assume that $s_{i}^{0}=s^{0}, s_{i}^{1}=s^{1}$, $\ldots, s_{i}^{2 l}=s^{2 l}$ do not depend upon $i$. Thus

$$
\begin{gathered}
s^{0} \cdot \mathcal{N}_{p_{i}^{0}} \cap \mathcal{N}_{p_{i}^{1}} \neq \emptyset, \\
\vdots \\
s^{2 l} \cdot \mathcal{N}_{p_{i}^{2 l}} \cap \mathcal{N}_{p_{i}^{0}} \neq \emptyset .
\end{gathered}
$$

By once again going to a subsequence of $\left(n_{i}\right)$, we may assume that there are $x^{0}=$ $x, x^{1}, \ldots, x^{2 l} \in 2^{\Gamma}$ such that $x^{k}\left|i=p_{i}^{k}\right| i$ for all $k \leq 2 l$ and all $i$. Thus for each $i$,

$$
\begin{aligned}
& s^{0} \cdot \mathcal{N}_{x^{0} \mid i} \cap \mathcal{N}_{x^{1} \mid i} \neq \emptyset, \\
& \vdots \\
& s^{2 l} \cdot \mathcal{N}_{x^{2 l} \mid i} \cap \mathcal{N}_{x^{0} \mid i} \neq \emptyset,
\end{aligned}
$$

therefore by the continuity of the shift action again,

$$
s^{0} \cdot x^{0}=x^{1}, s^{1} \cdot x^{1}=x^{2}, \ldots, s^{2 l} \cdot x^{2 l}=x^{0},
$$

i.e., $s^{2 l} s^{2 l-1} \ldots s^{1} s^{0} \cdot x=x$. Since $s^{2 l} s^{2 l-1} \ldots s^{1} s^{0} \neq 1$, we have $x \in 2^{\Gamma} \backslash X$. 
Remark 4.21. One can actually calculate quantitative upper bound estimates for $\frac{\left|X_{S, k, n}\right|}{\left|2^{F n}\right|}$ in the preceding theorem.

Let

$$
G_{S, k, n}=G_{S, n} \mid\left(2^{F_{n}} \backslash X_{S, k, n}\right)
$$

be the induced graph on $2^{F_{n}} \backslash X_{S, k, n}$. Then for $n$ large enough (depending upon $S, k$ ), $2^{F_{n}} \backslash X_{S, k, n} \neq \emptyset$ and $G_{S, k, n}$ is an ordinary graph, i.e., has no loops. Moreover, the odd girth of $G_{S, k, n}$ is bigger than $k$, i.e.,

$$
g_{\text {odd }}\left(G_{S, k, n}\right)>k .
$$

Furthermore, if $\delta_{S, k, n}=\frac{\left|X_{S, k, n}\right|}{\left|2^{F}\right|}$,

$$
i\left(G_{S, k, n}\right) \leq \frac{1}{1-\delta_{S, k, n}} i\left(G_{S, n}\right) \leq \frac{1}{1-\delta_{S, k, n}} i_{\mu}\left(S, s_{\Gamma}\right) .
$$

Let now $\Gamma=\mathbb{F}_{m}$ with free generating set $S_{m}=\left\{a_{1}, \ldots, a_{m}\right\}$, and let

$$
G_{m, k, n}=G_{S_{m}, k, n}, \delta_{m, k, n}=\delta_{S_{m}, k, n} .
$$

Then

$$
i\left(G_{m, k, n}\right) \leq \frac{1}{1-\delta_{m, k, n}} \cdot \frac{\sqrt{2 m-1}}{m+\sqrt{2 m-1}},
$$

and $\delta_{m, k, n} \rightarrow 0$ as $n \rightarrow \infty$. Thus we have a new family of explicitly given (finite) graphs with large odd girth and small independence ratio, thus large chromatic number. For example,

Theorem 4.22. Given $m, k$, for all large enough $n$ (depending upon $m, k$ ),

$$
\begin{aligned}
g_{\text {odd }}\left(G_{m, k, n}\right) & >k, \\
i\left(G_{m, k, n}\right) & \leq \frac{2 \sqrt{2 m-1}}{m+\sqrt{2 m-1}},
\end{aligned}
$$

and thus

$$
\chi\left(G_{m, k, n}\right) \geq \frac{m+\sqrt{2 m-1}}{2 \sqrt{2 m-1}} .
$$

(F) There are many other actions of $\mathbb{F}_{m}$ that exhibit phenomena similar to those discussed in $\S 4,(\mathbf{D}),(\mathbf{E})$ before.

(a) An action $a \in A(\Gamma, X, \mu)$ is called tempered if $\kappa_{0}^{a} \prec \lambda_{\Gamma}$ (see Kechris [22]). It is clear that for such a free action $a$, we have that $\left\|T_{S, \lambda_{\Gamma}}\right\|=\left\|T_{S, a}\right\|$, for any finite set of generators $S \subseteq \Gamma$ and thus for $\Gamma=\mathbb{F}_{m}$ we have estimates for $i_{\mu}(S, a), \chi_{\mu}^{\mathrm{ap}}(S, a)$ 
as in Theorem 4.17. Several examples of tempered actions of $\mathbb{F}_{m}$ are discussed in Kechris [22].

(b) It is shown in Lubotzky-Phillips-Sarnak [32] that there are free actions $a$ of $\mathbb{F}_{m}$, where $m=\frac{p+1}{2}$ with $p$ prime, by rotations on the sphere $S^{2}$, for which the norm $\left\|T_{S, a}\right\|$ is given by the Kesten formula, i.e., is equal to $\frac{2 \sqrt{p}}{p+1}$.

(c) Finally consider a countable, residually finite group $\Gamma$ and a sequence $\Gamma_{0}=$ $\Gamma \geq \Gamma_{1} \geq \cdots$ of decreasing normal subgroups which have finite index and $\bigcap_{n} \Gamma_{n}=$ $\{1\}$. Then the action of $\Gamma$ on the coset tree $T\left(\Gamma,\left(\Gamma_{n}\right)\right)$ gives rise to an action of $\Gamma$ on the boundary $\partial T\left(\Gamma,\left(\Gamma_{n}\right)\right)$ of this tree. We can view $\partial T\left(\Gamma,\left(\Gamma_{n}\right)\right)$ as a compact, metrizable, 0-dimensional group in which $\Gamma$ is naturally embedded as a dense subgroup (for details, see Kechris [24], Section 2) and this action is simply the translation action of $\Gamma$ on $\partial T\left(\Gamma,\left(\Gamma_{n}\right)\right)$, so it is free and ergodic. Denote this action by $a_{\Gamma,\left(\Gamma_{n}\right)}$. Let $S$ be a finite set of generators for $\Gamma$ and let $T_{S, a_{\Gamma,\left(\Gamma_{n}\right)}}=T_{S,\left(\Gamma_{n}\right)}$ be the corresponding averaging operator. Let also $H_{S, n}$ be the Cayley graph of $\Gamma / \Gamma_{n}$, with respect to the generators which are the images of those in $S$ under the canonical map of $\Gamma$ onto $\Gamma / \Gamma_{n}$. When the graphs $H_{S, n}$ are not bipartite, it is known (see Lubotzky-Zuk [34], 2.6, where however the assumption that $H_{S, n}$ are not bipartite is inadvertently left out) that the chain $\left(\Gamma_{n}\right)$ has property $(\tau)$ iff $\left\|T_{S,\left(\Gamma_{n}\right)}\right\|<1$. Thus in this case the independence number of $a_{\Gamma,\left(\Gamma_{n}\right)}$ is less than $1 / 2$. When some $H_{S, n}$ is bipartite, then the independence number of $a_{\Gamma,\left(\Gamma_{n}\right)}$ is $1 / 2$. It is not clear, e.g., in the case $\Gamma=\mathbb{F}_{m}$, what are the independence numbers of $a_{\Gamma,\left(\Gamma_{n}\right)}$, when $\left(\Gamma_{n}\right)$ does not have property $(\tau)$. Could they all be equal to $1 / 2$ ?

For certain free groups $\mathbb{F}_{m}$, one can actually construct $\left(\Gamma_{n}\right)$ as above for which the norm of the corresponding averaging operator is given by the Kesten formula (see Margulis [36], Lubotzky-Phillips-Sarnak [33], Morgenstern [40]). Note that for the actions $a_{\Gamma,\left(\Gamma_{n}\right)}$ the graphs $H_{S, n}$ are analogs of the finite graphs $G_{S, n}$ discussed in $\S 4$ (E) above. In the case of the constructions of the three papers mentioned above, these are Ramanujan graphs.

(G) Suppose $S$ is a finite set of generators for a group $\Gamma, m$ is a probability measure on $\Gamma$ supported by $S^{ \pm 1}$ with $m(\gamma)=m\left(\gamma^{-1}\right)$, and $\pi: \Gamma \rightarrow U(H)$ is a unitary representation. Then we can define again an averaging operator by

$$
T_{S, m, \pi}(f)=\sum_{s \in S^{ \pm 1}} m(s) \pi(s)(f)
$$

and for $a \in \operatorname{FR}(\Gamma, X, \mu)$ let

$$
T_{S, m, a}=T_{S, m, \kappa_{0}^{a}} .
$$

If $v=\left\|T_{S, m, a}\right\|$, then the argument in 4.16 goes through and shows that $i_{\mu}(S, a) \leq$ $\frac{v}{1+v}$.

Now it is easy to check that $\left(T_{S, m, a}\right)^{n}=T_{S^{n}, m^{* n}, a}$, where $m^{* n}$ is the $n$-fold convolution of $m$, defined by

$$
m^{* n}(\gamma)=\sum\left\{m\left(\gamma_{1}\right) \ldots m\left(\gamma_{n}\right): \gamma_{1} \ldots \gamma_{n}=\gamma\right\}
$$


It follows that if $\left\|T_{S, m, a}\right\|<1$, then

$$
\left\|T_{S^{n}, m^{* n}, a}\right\| \leq\left\|T_{S, m, a}\right\|^{n} \rightarrow 0
$$

as $n \rightarrow \infty$. It then follows that (exponentially) $i_{\mu}\left(S^{n}, a\right) \rightarrow 0$ as $n \rightarrow \infty$.

Take for example $\Gamma=\mathbb{F}_{2}$ with $S=\{a, b\}$ a free set of generators. Consider the graphs associated with (the free part of) the shift action $s_{\Gamma}$ on $2^{\Gamma}$ with respect to the set of generators $S^{2 n+1}(n \geq 1)$. Then (using as $m$ the normalized counting measure on $S^{ \pm 1}$, for which $\left.T_{S, m, s_{\Gamma}}=T_{S, s_{\Gamma}}\right)$ we see that $i_{\mu}\left(S^{2 n+1}, s_{\Gamma}\right) \rightarrow 0$ as $n \rightarrow \infty$. Moreover, there are no odd cycles in these graphs. We can then repeat the arguments in 4.19 and 4.20 to find another infinite family of finite graphs $G_{n, p, k}^{\prime}$ $(n, p, k \geq 1)$ such that for each $n, k$ and $p$ sufficiently large (depending upon $n$ and $k$ ), we have $g_{\text {odd }}\left(G_{n, p, k}^{\prime}\right)>k$ and $i\left(G_{n, p, k}^{\prime}\right)<\delta^{n}$, for some fixed constant $\delta<1$ (here $\delta=\left\|T_{S, s_{\Gamma}}\right\|^{2}$ ).

\section{On Brooks' Theorem}

Recall that for a graph $G=(X, E)$, we let $\Delta(G)$ denote $\sup \left\{d_{G}(x): x \in X\right\}$, where $d_{G}(x)=|\{y \in X: x E y\}|$. A point $x \in X$ is monovalent in $G$ if $d_{G}(x)=1$. In [26] it is shown that if $\Delta(G)$ is finite then $\chi_{B}(G) \leq \Delta(G)+1$. For finite graphs $G$, Brooks' Theorem states that actually $\chi(G) \leq \Delta(G)$, unless $G$ is an odd cycle or a complete graph. In this section we study Borel analogs of this bound.

Recall that $E^{*}$ is the equivalence relation generated by $E$, whose classes are called the connected components of $G$, and that $G$ is connected if $E^{*}$ has only one class. We abbreviate $[x]_{E^{*}}$ by $[x]_{G}$. For a cardinal $\kappa$, we say $G$ is $\kappa$-connected if $G \mid(X \backslash A)$ is connected for all $A \subseteq X$ with $|A|<\kappa$.

Also recall that we may view a graph $G$ as inducing a metric $\rho_{G}$ (informally called the $G$-distance) on each connected component of $G$ by setting $\rho_{G}\left(x_{0}, x_{1}\right)$ equal to one less than the length of the shortest path from $x_{0}$ to $x_{1}$, where a path is a sequence of vertices, each $G$-related to the next.

A graph $G$ on $X$ is vertex transitive if its automorphism group acts transitively on $X$. We say $G$ is weakly 3 -connected if there exist $x_{0}, x_{1} \in X$ such that $\rho_{G}\left(x_{0}, x_{1}\right)=2$ and $G \mid\left(X \backslash\left\{x_{0}, x_{1}\right\}\right)$ is connected. In the case that $G$ is vertex transitive and not a complete graph, this is stronger than 2-connectivity and weaker than 3-connectivity.

Theorem 5.1. Suppose that $G=(X, E)$ is a vertex-transitive Borel graph on a standard Borel space $X$ whose connected components are each weakly 3-connected. Suppose further that $\Delta(G)$ is finite. Then $\chi_{B}(G) \leq \Delta(G)$.

Proof. The argument is an amalgamation of the classical proof of Brooks' Theorem and the techniques involved in its analogue for approximate chromatic number (see Theorem 2.19). In addition to Lemma 2.18, we also require a technical lemma allowing us to find a nice subtree of $G$. 
Lemma 5.2. There is a Borel set $R \subseteq X$ and an acyclic Borel graph $T \subseteq G$, with vertex set $X$, such that

(1) no two distinct points of $R$ are within $G$-distance 3 ,

(2) each connected component of $T$ is finite,

(3) each connected component of $T$ contains exactly one point of $R$,

(4) each point in $R$ has two nonadjacent neighbors which are monovalent in $T$.

Granting this, we may prove the theorem. Fix $R$ and $T$ as in Lemma 5.2. We think of $R$ as a set of roots for the treed components of $T$. Let $X_{0}$ be the (Borel) set of neighbors of points in $R$ granted by item 4 of the lemma. Let then $X_{1} \subseteq X \backslash\left(X_{0} \cup R\right)$ be those points monovalent in $T \mid\left(X \backslash X_{0}\right)$ and generally let $X_{i} \subseteq X \backslash\left(X_{0} \cup \cdots \cup\right.$ $\left.X_{i-1} \cup R\right)$ be those points monovalent in $T \mid\left(X \backslash\left(X_{0} \cup \cdots \cup X_{i-1}\right)\right)$. Item 2 of the lemma ensures that $X=R \sqcup \bigsqcup_{i \in \mathbb{N}} X_{i}$.

As $X_{0}$ is a $G$-independent set (by item 1 ), we may initially color every point in $X_{0}$ with color 0 . Since every element of $X_{1}$ is adjacent to something closer (with respect to $\left.\rho_{T}\right)$ to $R$, each point in $X_{1}$ has degree less than $\Delta(G)$ in the restriction $G \mid\left(X_{0} \cup X_{1}\right)$. Lemma 2.18 then allows us to extend our coloring to a Borel $\Delta(G)$ coloring of $X_{0} \cup X_{1}$. Proceeding in this fashion, we extend our coloring in turn to each $X_{i}$ until we have a Borel $\Delta(G)$-coloring of $X \backslash R$.

To complete the coloring, we simply need to choose colors for points in $R$. But each such point sees at most $\Delta(G)$ neighbors, and at least two of the neighbors receive color 0 , so we may assign it the least color unused by its neighbors.

Proof of Lemma 5.2. For convenience, fix some $x \in X$. Since $G$ is weakly 3-connected, we may find nonadjacent neighbors $y_{0}, y_{1}$ of $x$ such that $G \mid\left([x]_{G} \backslash\left\{y_{0}, y_{1}\right\}\right)$ is connected. Fix $r \geq 2$ sufficiently large so that $G \mid\left(B_{r}(x) \backslash\left\{y_{0}, y_{1}\right\}\right)$ is connected, where $B_{r}(x)$ denotes the $\rho_{G}$-ball of radius $r$ about $x$. We may then fix a spanning tree $H$ of $G \mid\left(B_{r}(x) \backslash\left\{y_{0}, y_{1}\right\}\right)$ and subsequently extend it to a spanning tree $H^{\prime}$ of $G \mid B_{r}(x)$ by connecting $y_{0}$ and $y_{1}$ to $x$ (leaving them monovalent).

Now, let $R$ be a Borel maximal $G^{2 r}$-independent subset of $X$, where $G^{2 r}$ is the graph relating two distinct points $x_{0}, x_{1}$ if $\rho_{G}\left(x_{0}, x_{1}\right) \leq 2 r$. That is, no two distinct points of $R$ are within $G$-distance $2 r$, but every element of $X$ is within $G$-distance $2 r$ of something in $R$. Since for every $x_{0}, x_{1} \in R, B_{r}\left(x_{0}\right) \cap B_{r}\left(x_{1}\right)=\emptyset$, we may "copy" in a Borel way $H^{\prime}$ onto each element of $R$ to obtain an acyclic graph $T^{\prime} \subseteq G$ connecting every element of $B_{r}(R)=\left\{x: \rho_{G}(x, R) \leq r\right\}$ to its nearest element of $R$.

We may extend this to an acyclic graph connecting every element of $B_{r+1}(R)=$ $\left\{x: \rho_{G}(x, R) \leq r+1\right\}$ to exactly one element of $R$ by connecting each element of $B_{r+1}(R) \backslash B_{r}(R)$ to one of its neighbors in $B_{r}(R)$. Continuing, we may extend step by step until we have an acyclic graph $T$ connecting every element of $B_{2 r}(R)$ to exactly one element of $R$. Since $B_{2 r}(R)=X$, we are done once we know each connected component of $T$ is finite. But since $[x]_{T} \subseteq B_{2 r}(x)$ for all $x \in R$, each connected component of $T$ must have cardinality at most $\Delta(G)^{2 r}$. 
We spend the remainder of the section discussing graphs for which the hypotheses of Theorem 5.1 are met. Towards this end, we must recall some notions arising naturally in the study of connectivity of infinite graphs [21]. Given a graph $G=$ $(X, E)$ and a subset $F \subseteq X$, we let $\partial F$ denote the (external) boundary of $F$, defined as $\{x \in X \backslash F: \exists y \in F(x E y)\}$. In the notation of the proof of Theorem 5.1 we then have $\partial F=B_{1}(F) \backslash F$. We denote by $F^{e}$ the exterior of $F$, defined as $X \backslash(F \cup \partial F)$. Equivalently, $F^{e}=X \backslash B_{1}(F)$.

Our first goal is a self-contained proof of the following:

Proposition 5.3 ([21]). Suppose that $G=(X, E)$ is an infinite, connected, vertextransitive graph with finite $\Delta(G) \geq 3$ and that $F$ is a nonempty, finite subset of $X$. Then $|\partial F| \geq 3$.

Proof. We let $\kappa_{f}$ denote the smallest possible cardinality of a boundary of a finite nonempty set of vertices of $G$. That is,

$$
\kappa_{f}=\min \{|\partial F|: F \subseteq X \text { finite and nonempty }\} .
$$

A fragment is a finite set $F$ with $|\partial F|=\kappa_{f}$. Since $F \subseteq\left(F^{e}\right)^{e} \subseteq F \cup \partial F$ and also $\partial\left(\left(F^{e}\right)^{e}\right) \subseteq \partial F$, we have that $\left(F^{e}\right)^{e}=F$ whenever $F$ is a fragment.

Suppose now that $F_{1}$ and $F_{2}$ are two fragments. We have

$$
\begin{aligned}
\mid \partial\left(F_{1}\right. & \left.\cap F_{2}\right)|+| \partial\left(F_{1} \cup F_{2}\right) \mid \\
& =\left|\partial\left(F_{1} \cap F_{2}\right) \cap \partial\left(F_{1} \cup F_{2}\right)\right|+\left|\partial\left(F_{1} \cap F_{2}\right) \cup \partial\left(F_{1} \cup F_{2}\right)\right| \\
& \leq\left|\partial F_{1} \cap \partial F_{2}\right|+\left|\partial F_{1} \cup \partial F_{2}\right| \\
& =\left|\partial F_{1}\right|+\left|\partial F_{2}\right| \\
& =2 \kappa_{f} .
\end{aligned}
$$

In particular, if $F_{1} \cap F_{2}$ is nonempty, it must be a fragment. We may therefore unambiguously define an atom as a minimal under inclusion fragment, noting that distinct atoms are disjoint. By transitivity, it follows that the atoms of $G$ partition $X$.

It is therefore enough to show that $|\partial F| \geq 3$ for some atom $F$. Suppose that $F_{1}$ and $F_{2}$ are distinct atoms with adjacent vertices $x_{1} E x_{2}$ such that $x_{1} \in F_{1}$ and $x_{2} \in F_{2}$. By reasoning as above, we see

$$
\begin{aligned}
\mid \partial\left(F_{1}\right. & \left.\cap F_{2}^{e}\right)|+| \partial\left(F_{1}^{e} \cap F_{2}\right) \mid \\
& =\left|\partial\left(F_{1} \cap F_{2}^{e}\right) \cap \partial\left(F_{1}^{e} \cap F_{2}\right)\right|+\left|\partial\left(F_{1} \cap F_{2}^{e}\right) \cup \partial\left(F_{1}^{e} \cap F_{2}\right)\right| \\
& \leq\left|\partial F_{1} \cap \partial F_{2}\right|+\left|\partial F_{1} \cup \partial F_{2}\right| \\
& =\left|\partial F_{1}\right|+\left|\partial F_{2}\right| \\
& =2 \kappa_{f} .
\end{aligned}
$$

That is, if both $F_{1} \cap F_{2}^{e}$ and $F_{1}^{e} \cap F_{2}$ were nonempty, then they would both be fragments. In particular, $F_{1} \cap F_{2}^{e}=F_{1}$, i.e., $F_{1} \subseteq F_{2}^{e}$, contradicting the fact that 
$F_{1} \cap \partial F_{2} \neq \emptyset$. Without loss of generality, we may assume $F_{1}^{e} \cap F_{2}$ is empty, and thus $F_{2} \subseteq \partial F_{1}$. Certainly $\partial F_{1}$ cannot equal $F_{2}$ or (by transitivity) every atom would have a single atom as its boundary, forcing the graph to be finite (the union of at most two atoms). Thus $\left|\partial F_{1}\right| \geq\left|F_{2}\right|+1$, which gives the desired bound as long as $\left|F_{2}\right|>1$. But, of course, if $\left|F_{2}\right|=1$, then $\left|\partial F_{2}\right|=\Delta(G) \geq 3$ as required.

Remark 5.4. A more detailed investigation into the nature of fragments gives much more information about the connectivity of infinite graphs; see [21] for more details.

We will also need to borrow from the study of ends of a graph (see, e.g., [37], 11.4). Recall that we say a connected, locally finite graph $G(X, E)$ has at most $n$ ends $(n \geq 0)$ if for all finite $F \subseteq X$ the induced subgraph $G \mid(X \backslash F)$ has at most $n$ infinite connected components. Then $G$ has $n$ ends if $n$ is least such that $G$ has at most $n$ ends. If no such $n$ exists, we say $G$ has infinitely many ends. We may view the number of ends of $G$ as the limit of the number of infinite components of $G \mid\left(X \backslash F_{i}\right)$, where $F_{0} \subseteq F_{1} \subseteq \cdots$ is an exhaustive sequence of finite subsets of $X$.

Recall that an connected, infinite, vertex-transitive graph has either one, two, or infinitely many ends (see [15], F64, p. 497). In this situation, knowing the number of ends of a graph can give information about its connectivity.

Proposition 5.5. Suppose that $G=(X, E)$ is an infinite, connected, vertex-transitive graph with finite $\Delta(G)$ and assume that $G$ has one end. Then $G$ is 3-connected.

Proof. Note that since $G$ has one end, $\Delta(G) \geq 3$. Fix $F \subseteq X$ with $|F| \leq 2$. By Proposition 5.3, $G \mid(X \backslash F)$ has no finite connected components. Since $G$ has one end, it follows that $G \mid(X \backslash F)$ is connected.

On the other hand, knowledge of a graph's connectivity can translate into knowledge of its ends.

Proposition 5.6. Suppose that $G=(X, E)$ is an infinite, connected, vertex-transitive graph with $\Delta(G) \geq 3$ which is not 2-connected. Then $G$ has infinitely many ends.

Proof. By transitivity, we have that $G \mid(X \backslash\{x\})$ is disconnected for every $x \in X$. Fix $x_{0}, x_{1}$ with $\rho_{G}\left(x_{0}, x_{1}\right) \geq 2$. Deleting $x_{0}$ results in at least two connected components, and further deleting $x_{1}$ splits its component into at least two subcomponents. Since Proposition 5.3 ensures that none of the components of $G \mid\left(X \backslash\left\{x_{0}, x_{1}\right\}\right)$ is finite, $G \mid\left(X \backslash\left\{x_{0}, x_{1}\right\}\right)$ has at least three infinite components. Thus, $G$ has infinitely many ends.

Recall that if $\Gamma$ is a group with finite generating set $S$, the number of ends of $\operatorname{Cay}(\Gamma, S)$ is independent of the choice of $S$ (see, e.g., [37], 11.4). We may thus say $\Gamma$ has $n$ ends exactly when $\operatorname{Cay}(\Gamma, S)$ has $n$ ends (and similarly with infinitely many ends). 
Proposition 5.7. Suppose that $\Gamma$ is a group with finite generating set $S$. Suppose further that $\Gamma$ has two ends and is isomorphic neither to $\mathbb{Z}$ nor to $(\mathbb{Z} / 2 \mathbb{Z}) *(\mathbb{Z} / 2 \mathbb{Z})$. Then $\operatorname{Cay}(\Gamma, S)$ is weakly 3-connected.

Proof. It is well known that $\Gamma$ has a finite index subgroup isomorphic to $\mathbb{Z}$ (see, e.g., [37], 11.4), a fact that we will use repeatedly below. In particular, this implies that every subgroup of $\Gamma$ isomorphic to $\mathbb{Z}$ is of finite index. Moreover, there is an element $z \in \Gamma$ of infinite order such that either $z \in S$ or $z$ is the product of two elements of $S$ (see [45], p. 25, or [19]). Fix such a $z$ such that $z^{2} \notin S$.

Lemma 5.8. There is some fixed $N_{z}$ such that any vertex of $\mathrm{Cay}(\Gamma, S)$ is connected to an element of $\langle z\rangle$ by a path of length at most $N_{z}$.

Proof. There must be a finite index subgroup $\Gamma_{0} \leq\langle z\rangle$ such that $\Gamma_{0}$ is normal in $\Gamma$. Let $N_{z}=\left[\Gamma: \Gamma_{0}\right]$.

Now fix $x \in \Gamma$. Working in the quotient group $\Gamma / \Gamma_{0}$, there is a way of writing $x \Gamma_{0}$ as a word of the form $\left(s_{1} \Gamma_{0}\right)\left(s_{2} \Gamma_{0}\right) \ldots\left(s_{k} \Gamma_{0}\right)$ with each $s_{i} \in S$ and $k \leq N_{z}$. Now working in the Cayley graph $\operatorname{Cay}(\Gamma, S)$, we see that the path $\left(x, s_{1}^{-1} x, s_{2}^{-1} s_{1}^{-1} x, \ldots, s_{k}^{-1} \ldots s_{1}^{-1} x\right)$ connects $x$ to some element of $\Gamma_{0}$, which is necessarily in $\langle z\rangle$.

Suppose first that $z \in S$. The right cosets $\{\langle z\rangle a: a \in \Gamma\}$ partition the vertices of Cay $(\Gamma, S)$ into finitely many sets, the graph's restriction to each resembling the Cayley graph of the integers.

Claim 5.9. If $F \subseteq \Gamma$ is finite such that $\operatorname{Cay}(\Gamma, S) \mid(\Gamma \backslash F)$ has two infinite connected components, then $F$ meets every right coset of $\langle z\rangle$.

Proof. By homogeneity, it is enough to prove that $F$ meets $\langle z\rangle$. Assume not, towards a contradiction. If $x$ has distance $>N_{z}$ from $F$, then 5.8 gives a path from $x$ to $\langle z\rangle$ disjoint from $F$, so $x$ is in the same component of $\operatorname{Cay}(\Gamma, S) \mid(\Gamma \backslash F)$ as $\langle z\rangle$, thus $\operatorname{Cay}(\Gamma, S) \mid(\Gamma \backslash F)$ has a unique infinite component, a contradiction.

Recall that by Proposition 5.3, the deletion of two vertices of Cay $(\Gamma, S)$ cannot result in a finite connected component. If we set $F_{0}=\left\{z, z^{-1}\right\}$, then $F_{0}$ meets only one right coset of $\langle z\rangle$. Since $\Gamma$ is not isomorphic to $\mathbb{Z}$, we may conclude from the claim that $\operatorname{Cay}(\Gamma, S) \mid\left(\Gamma \backslash F_{0}\right)$ is connected, and thus $\operatorname{Cay}(\Gamma, S)$ is weakly 3-connected.

It remains to handle the case that no element of $S$ has infinite order. Recall then that $z$ is the product of two distinct elements of $S$, say $z=s t$. We have a slightly weaker analog of the previous claim.

Claim 5.10. If $F \subseteq \Gamma$ is finite such that $\operatorname{Cay}(\Gamma, S) \mid(\Gamma \backslash F)$ has two infinite connected components, then $F \cup s F$ meets every right coset of $\langle s t\rangle$. 
Proof. As before, each infinite connected component of $\mathrm{Cay}(\Gamma, S) \mid(\Gamma \backslash F)$ meets each right coset of $\langle s t\rangle$. Then for each right coset $\langle s t\rangle a$, we have $b \in\langle s t\rangle a$ and a path of the form $\left(b, t b, s t b, t s t b, \ldots,(s t)^{k} b\right)$ in $\operatorname{Cay}(\Gamma, S)$ such that $b$ and $(s t)^{k} b$ are in distinct components of $\operatorname{Cay}(\Gamma, S) \mid(\Gamma \backslash F)$. This means that some vertex $x$ in the path must be an element of $F$.

If $x$ is of the form $(s t)^{i} b$, then $x \in\langle s t\rangle a$. On the other hand, if $x$ is of the form $t(s t)^{i} b$, then $s x \in\langle s t\rangle a$. Thus, $F \cup s F$ meets $\langle s t\rangle a$.

We now set $F_{0}=\left\{t^{-1}, s\right\}$, and will show that $\operatorname{Cay}(\Gamma, S) \mid\left(\Gamma \backslash F_{0}\right)$ is connected. Suppose towards a contradiction that it is not connected; then by Proposition 5.3 it must have two infinite components. We see that $F_{0}$ meets a single right coset of $\langle s t\rangle$, namely $\langle s t\rangle s$. On the other hand, $s F$ meets at most two other right cosets, $\langle s t\rangle s^{2}$ and $\langle s t\rangle s t^{-1}=\langle s t\rangle t^{-2}$. By the last claim, the union of these cosets must be the entire group (in particular $\Gamma=\langle s, t\rangle$ ), and so the identity must fall into one. The three equations

$$
\begin{aligned}
& (s t)^{n}=s^{-1}, \\
& (s t)^{n}=s^{-2},
\end{aligned}
$$

and

$$
(s t)^{n}=t^{2}
$$

have solutions only when $n=0$, otherwise the left-hand side has infinite order while the right-hand side has finite order. We conclude that at least one of $s$ and $t$ has order 2 . Replacing $s t$ by $t^{-1} s^{-1}$ if necessary, we may assume without loss of generality that $t^{2}=1$.

Then $\Gamma=\langle s t\rangle \cup\langle s t\rangle s \cup\langle s t\rangle s^{2}$, ensuring that $s$ has order at most 3. If $s$ has order 2 , then $\Gamma=(\mathbb{Z} / 2 \mathbb{Z}) *(\mathbb{Z} / 2 \mathbb{Z})$, which is precluded by our hypothesis. Thus we may assume $s$ has order 3 .

Arguing as above, the right cosets $\langle s t\rangle t,\langle s t\rangle s t$ and $\langle s t\rangle s^{2} t$ are disjoint and cover $\Gamma$. It is clear that $\langle s t\rangle=\langle s t\rangle$ st and $\langle s t\rangle t=\langle s t\rangle s$, so $\langle s t\rangle s^{2}=\langle s t\rangle s^{2} t$. The equation

$$
(s t)^{n} s^{2}=s^{2} t .
$$

has a solution only when $n=0$, since $s^{2} t s^{-2}$ has finite order. Thus $s^{2}=s^{2} t$ and consequently $t$ is the identity, a contradiction.

We finally apply these results to build a class of graphs satisfying Brooks' Theorem in the Borel context.

Theorem 5.11. Suppose that $G$ is a vertex-transitive Borel graph on a standard Borel space $X$ with $\Delta(G)$ finite and whose connected components each have one end. Then $\chi_{B}(G) \leq \Delta(G)$.

Proof. By Proposition 5.5, each connected component of $G$ is 3-connected, and thus weakly 3-connected. Therefore, the hypotheses of Theorem 5.1 are met. 
Theorem 5.12. Suppose that $\Gamma$ is a countable infinite group isomorphic neither to $\mathbb{Z}$ nor to $(\mathbb{Z} / 2 \mathbb{Z}) *(\mathbb{Z} / 2 \mathbb{Z})$. Suppose further that $\Gamma$ has finitely many ends. Let $S$ be a finite set of generators for $\Gamma$ and put $d=\left|S^{ \pm 1}\right|$. Then for any free Borel action $A$ of $\Gamma$ on a standard Borel space $X$, we have $\chi_{B}(S, A) \leq d$.

Proof. If $\Gamma$ has one end, this is a consequence of Theorem 5.11. If $\Gamma$ has two ends, Proposition 5.7 ensures that $G(S, A)$ meets the hypotheses of Theorem 5.1.

Remark 5.13. The assumption that $\Gamma$ is neither $\mathbb{Z}$ nor $(\mathbb{Z} / 2 \mathbb{Z}) *(\mathbb{Z} / 2 \mathbb{Z})$ is necessary. If $\Gamma$ is either of those groups equipped with its natural generating set $S$ (with $\left|S^{ \pm 1}\right|=2$ ), then the free part of the shift action $s_{\Gamma}$ of $\Gamma$ on $2^{\Gamma}$ has Borel chromatic number 3 .

Example 5.14. Finitely generated groups that have only finitely many ends, and thus Theorem 5.12 applies, include: Property (T) groups (see, e.g., [42]); groups of cost 1 (see Gaboriau [11]), and thus, in particular, amenable groups, direct products of two infinite groups, etc.; and groups not containing $\mathbb{F}_{2}$ (by Stallings' Theorem, see [44]). For a finitely generated torsion-free group $\Gamma$, Stallings' Theorem implies that $\Gamma$ has infinitely many ends iff $\Gamma$ is a non-trivial free product. So in this case 5.12 holds for any group that is not a non-trivial free product.

Example 5.15. Suppose now that $\Gamma$ has a generating set $S$ of cardinality 2 and finitely many ends but is not isomorphic to $\mathbb{Z}$ or $(\mathbb{Z} / 2 \mathbb{Z}) *(\mathbb{Z} / 2 \mathbb{Z})$. Then for any free Borel action $A$ of $\Gamma$ which admits an invariant Borel probability measure with respect to which it is weakly mixing, we have $\chi_{B}(S, A) \in\{3,4\}$. In particular, this holds for the free part of the shift action of $\Gamma$ on $2^{\Gamma}$. This generalizes a theorem of Gao-Jackson [12] and Miller, who proved this for $\Gamma=\mathbb{Z}^{2}$ (see $\S 4$, (D)).

Example 5.16. In Aldous-Lyons [4], 10.5, it is pointed out that for any sofic group $\Gamma$ and finite generating set $S$, there is a free, measure preserving action $a$ of $\Gamma$ with $\chi_{\mu}(S, a) \leq d=\left|S^{ \pm 1}\right|$.

It is still unknown whether the Brooks bound holds for groups with infinitely many ends, even in the torsion-free context. In fact, the following question remains unanswered.

Question 5.17. Does every graph corresponding to a free Borel action of $\mathbb{F}_{n}(n \geq 2)$, with a free set of generators, admit a Borel coloring with $2 n$ colors?

(Addendum. Andrew Marks has now given a negative answer to this question.) 


\section{A matching problem}

Consider a Borel bipartite graph $G=(X, E)$, i.e., $X=X_{1} \sqcup X_{2}$ is a Borel partition and if $(x, y) \in E$ then one of $x, y$ is in $X_{1}$ and the other is in $X_{2}$. If $d(x)=k<\boldsymbol{\aleph}_{0}$ for every $x \in X$, then by a theorem of König (a special case of Hall's Theorem), $G$ admits a matching, i.e., a bijection $\varphi: X_{1} \rightarrow X_{2}$ such that $(x, \varphi(x)) \in E, \forall x \in X$. The question was raised (see, e.g., Miller [38]) whether there is a Borel version of that theorem, more precisely, whether there is a Borel matching.

Laczkovich [30] provided the following counterexample for $k=2$.

Fix an irrational $0<\alpha<1$ and consider the set $R$ consisting of the following rectangle inscribed in the unit square, together with the indicated two corner points.

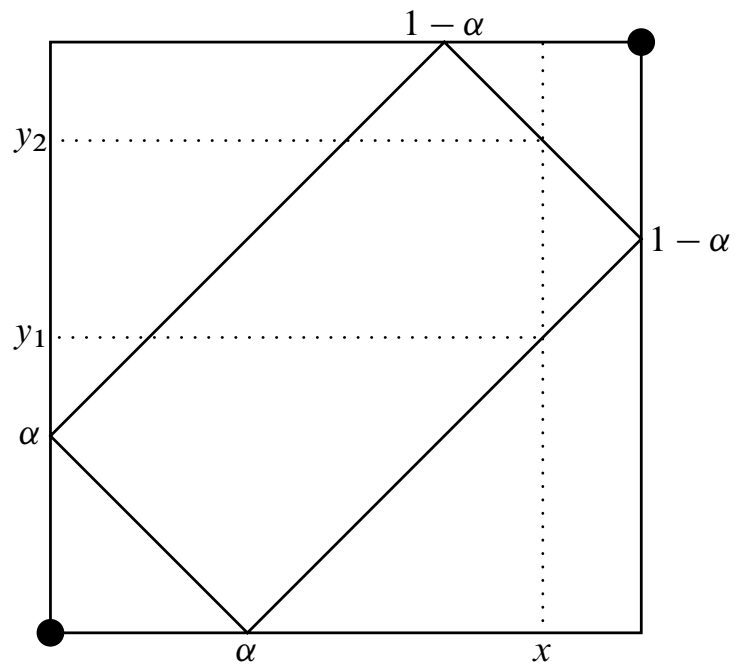

We take $X_{1}, X_{2}$ to be two disjoint copies of $[0,1]$ and for $x \in X_{1}$ its two neighbors $y_{1}, y_{2} \in X_{2}$ are such that $\left(x, y_{i}\right) \in R$. The two neighbors of any $y \in X_{2}$ are defined analogously. Clearly this is a Borel graph in which every vertex has degree 2, but Laczkovich showed that it does not have a Borel matching.

In the paper Kłopotowski-Nadkarni-Sarbadhikari-Srivastava [29], the authors argue that the graph shown below which consists of 4 "copies" of the preceding graph (actually, the authors discard finitely many connected components rather than adding dots at the corners, but it is clear that one graph admits a Borel matching if and only if the other does), and in which every vertex has degree 4 provides a counterexample for $k=4$ (and similarly for all even $k$ ). They also raised the question of whether there is a counterexample for $k=3$. 

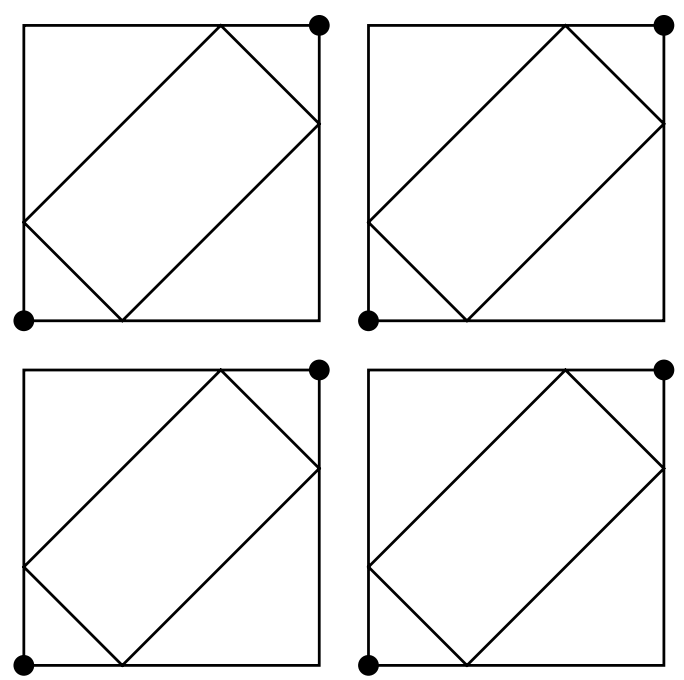

Lyons (private communication) showed that the above example actually does not work, as it has a Borel matching. A simpler argument is as follows:
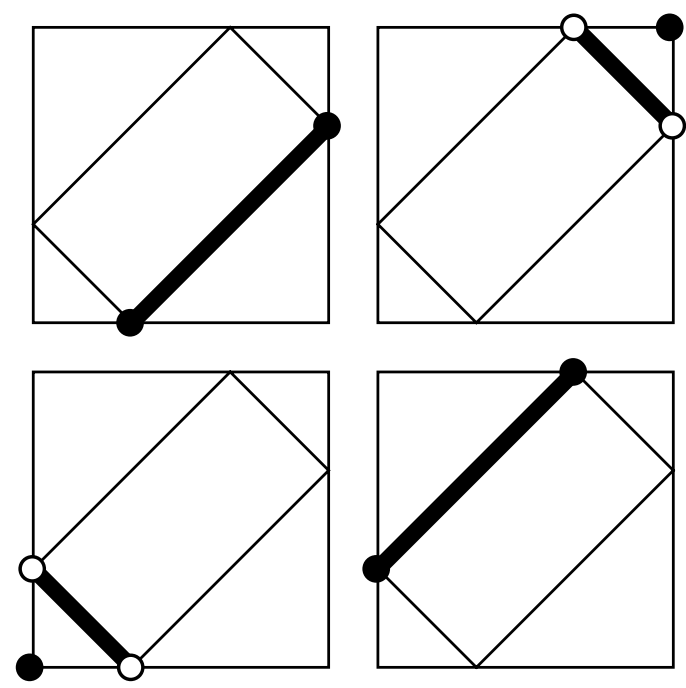

The boldface segments and dots provide the matching, where as usual an endpoint of a segment is colored black if it is included, and is colored white if it is not included.

However, it turns out that there is a way to modify this construction to find counterexamples for every even $k$. For example, for $k=4$, the idea is to construct a 
"Sudoku" version which is illustrated in the following picture:

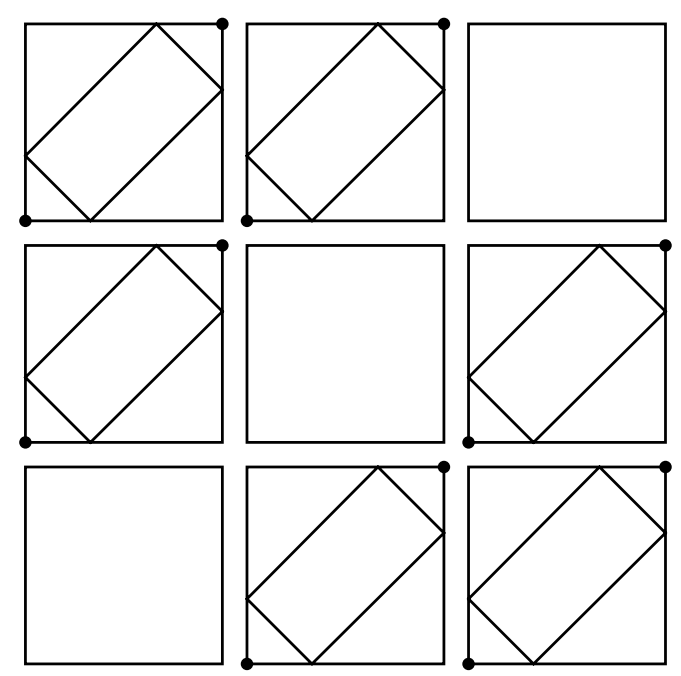

Let us give a detailed argument. Fix a Borel bipartite graph $\left(X_{1} \sqcup X_{2}, E\right)$ with degree $k=2$ possessing no Borel matching. Define from this a new graph $\left(\bar{X}_{1} \sqcup\right.$ $\left.\bar{X}_{2}, \bar{E}\right)$ as follows: $\bar{X}_{1}=X_{1} \times\{1,2,3\}, \bar{X}_{2}=X_{2} \times\{1,2,3\}$, and $(x, i) \bar{E}(y, j) \Leftarrow$ $\Rightarrow(i \neq j$ and $x E y)$. This has degree $k=4$ and it is enough to show that if there is a Borel injection $\bar{f}: \bar{X}_{1} \rightarrow \bar{X}_{2}$ such that $(x, i) \bar{E} \bar{f}(x, i)$, then there is a Borel injection $f: X_{1} \rightarrow X_{2}$ with $x E f(x)$ (and similarly if we switch the roles of $\left.\bar{X}_{1}, \bar{X}_{2}\right)$. Granting this, if there is a Borel matching for $\left(\bar{X}_{1} \sqcup \bar{X}_{2}, \bar{E}\right)$, there are two Borel injections, from $X_{1}$ to $X_{2}$ and vice versa, whose graphs are contained in $E$, so, by a Schröder-Bernstein argument, there is a Borel matching for $\left(X_{1} \sqcup X_{2}, E\right)$, a contradiction.

So fix $\bar{f}$ as above, which we will use to define $f$. Given $x \in X$, consider $\bar{f}(x, 1)=(u, a), \bar{f}(x, 2)=(v, b)$, and $\bar{f}(x, 3)=(w, c)$. Then $x E u, x E v$, and $x E w$. Since $\left(X_{1} \sqcup X_{2}, E\right)$ has degree 2 , at least two of $u, v, w$ are equal. So there is a unique $y \in X_{2}$ such that for at least two distinct $i, j \leq 3$, we have $\bar{f}(x, i)=(y, k)$, $\bar{f}(x, j)=(y, l)$ (for some necessarily distinct $k, l)$. Put $f(x)=y$; we claim that this works. To see this, take $x \neq x^{\prime}$. If $f(x)=f\left(x^{\prime}\right)=y$, then let $i \neq j$ be such that $\bar{f}(x, i)=(y, k), \bar{f}(x, j)=(y, l)$ and let $i^{\prime} \neq j^{\prime}$ be such that $\bar{f}\left(x^{\prime}, i^{\prime}\right)=\left(y, k^{\prime}\right)$, $\bar{f}\left(x^{\prime}, j^{\prime}\right)=\left(y, l^{\prime}\right)$. As before, $k \neq l$ and $k^{\prime} \neq l^{\prime}$. It follows that one of $k, l$ is equal to one of $k^{\prime}, l^{\prime}$, contradicting the injectivity of $\bar{f}$.

The same proof works for degree $k=6$ by dropping from the definition of $\bar{E}$ the condition $i \neq j$ (i.e., in the preceding picture inscribing the rectangle into all nine of the small squares). In general, for degrees $k=4 n$ and $k=4 n+2(n \geq 1)$ one uses the same argument with the $(2 n+1) \times(2 n+1)$ square. 
As far as we know, the case $k=3$ is open. (Addendum. Andrew Marks has now constructed examples for every $k \geq 2$.) We sketch below an alternative approach to the $k=2$ case which adapts naturally to the $k=3$ case, relating the question of whether a bipartite graph has no Borel matching to the calculation of the independence number associated with the shift action of an appropriate group. This was actually for us a motivation for looking at the independence number of such graphs.

Let $m \geq 2$ and $A=\left\{1, a, a^{2}, \ldots, a^{m-1}\right\}$ and $B=\left\{1, b, b^{2}, \ldots, b^{m-1}\right\}$ be two copies of the cyclic group of order $m$. Let $\Gamma_{m}=A * B$ and consider the shift action of $\Gamma_{m}$ on $2^{\Gamma_{m}}$, and let $Y \subseteq 2^{\Gamma_{m}}$ be its free part. Let $X_{1}=Y / A$, the set of $A$-orbits under the shift action, and $X_{2}=Y / B$. Then $X_{1}$ and $X_{2}$ are standard Borel spaces and let $X=X_{1} \sqcup X_{2}$. Define the bipartite graph $G_{m}=(X, E)$ by

$$
p E q \Longleftrightarrow p \cap q \neq \varnothing \text {. }
$$

If $p \in X_{1}, q \in X_{2}$ and $p \cap q \neq \emptyset$, then for some $y \in p \cap q, p=A \cdot y$ and $q=B \cdot y$. Since the action of $\Gamma$ on $Y$ is free, clearly $p \cap q=\{y\}$. Thus there is a canonical bijective correspondence between $Y$ and $E$, namely

$$
y \mapsto\{A \cdot y, B \cdot y\}
$$

(we view $E$ here as a set of unordered pairs). Clearly each vertex in $G_{m}$ has degree exactly $m$.

Suppose now that $f: X_{1} \rightarrow X_{2}$ is a Borel matching for $G$. By the above identification, $f$ can be viewed as a Borel subset $M \subseteq Y$ and the condition of being a matching corresponds exactly to the assertion that $M$ meets every $A$-orbit in exactly one point, and likewise meets every $B$-orbit. That is, $M$ is a common transversal for the $A$ - and $B$-orbits.

The set $S=(A \cup B) \backslash\{1\} \subseteq \Gamma_{m}$ is a set of generators for $\Gamma_{m}$ and the above condition for $M$ implies that $M$ is an independent Borel set for the graph $G\left(S, s_{\Gamma_{m}}\right)$. Moreover it is clear that for the product measure $\mu$ on $2^{\Gamma_{m}}, \mu(M)=1 / m$. Thus, in particular, if there is a Borel matching in $G_{m}=(X, E)$, then $i_{\mu}\left(S, s_{\Gamma_{m}}\right) \geq 1 / \mathrm{m}$.

On the other hand, if $i_{\mu}\left(S, s_{\Gamma_{m}}\right) \geq 1 / m$ and the supremum is attained, say by an independent Borel set $C$, then $C$ must meet almost every $A$-orbit and almost ever $B$-orbit in exactly one point. It follows that the existence of an almost everywhere Borel matching in $G_{m}$ is equivalent to the statement that $i_{\mu}\left(S, s_{\Gamma_{m}}\right)=1 / m$ and the supremum is attained.

If $m=2$ this is impossible: since the action $s_{\Gamma_{2}}$ is weakly mixing, there can be no independent set of measure $1 / 2$. Thus there is no Borel matching in the graph $G_{2}$, providing an alternate proof of Laczkovich's theorem. In fact, there is no almost everywhere Borel matching in $G_{2}$.

We do not know whether there is a Borel matching for $G_{3}$. (Addendum. Andrew Marks has shown that this has a negative answer if one replaces $2^{\Gamma_{3}}$ by $3^{\Gamma_{3}}$.) In an earlier version of this paper, we have asked whether there is even an almost everywhere Borel matching in $G_{3}$. However, Lyons and Nazarov [35] have now shown that this is 
indeed the case, or equivalently that $i_{\mu}\left(S, s_{\Gamma_{3}}\right)=1 / 3$ and the supremum is attained. From this it follows that in fact $i_{\mu}(S, a)=1 / 3$ for any $a \in \operatorname{FR}\left(\Gamma_{3}, X, \mu\right)$. That $i_{\mu}(S, a) \leq 1 / 3$ is clear since any independent set contains at most one element in each $A$-orbit. Since $s_{\Gamma_{3}} \prec a$ we also have the reverse inequality. It also follows that $\chi_{\mu}^{\mathrm{ap}}(S, a)=3$. It is enough to prove it for $a=s_{\Gamma}$. To see this let $T$ be a set meeting each triangle in exactly one point, a.e.. This is one color. Removing $T$ we get an acyclic degree 2 graph, so two colors are enough up to any $\varepsilon$.

\section{References}

[1] M. Abért and G. Elek, Dynamical properties of profinite actions. Ergodic Theory Dynam. Systems 32 (2012), 1805-1835.

[2] M. Abért, A. Jaikin-Zapirain, and N. Nikolov, The rank gradient from a combinatorial viewpoint. Groups Geom. Dyn. 5 (2011), 213-230. Zbl 06010056 MR 2782170

[3] M. Abért and B. Weiss, Bernoulli actions are weakly contained in any free action. Ergodic Theory Dynam. Systems, to appear; Doi 10.1017/S0143385711000988.

[4] D. Aldous and R. Lyons, Processes on unimodular random networks. Electron. J. Probab. 12 (2007), no. 54, 1454-1508. Zbl 1131.60003 MR 2354165

[5] B. Bekka, P. de la Harpe, and A. Valette, Kazhdan's property (T). New Math. Monogr. 11, Cambridge University Press, Cambridge 2008. Zbl 1146.22009 MR 2415834

[6] L. Bowen, Periodicity and circle packings of the hyperbolic plane. Geom. Dedicata 102 (2003), 213-236. Zbl 1074.52007 MR 2026846

[7] L. Bowen, Weak isomorphisms between Bernoulli shifts. Israel J. Math. 183 (2011), 93-102. Zbl 05942609 MR 2811154

[8] C. T. Conley and B. D. Miller, An antibasis result for graphs of infinite Borel chromatic number. Proc. Amer. Math. Soc., to appear.

[9] G. Davidoff, P. Sarnak, and A. Valette, Elementary number theory, group theory, and Ramanujan graphs. London Math. Soc. Student Texts 55, Cambridge University Press, Cambridge 2003. Zbl 1032.11001 MR 1989434

[10] R. Diestel, Graph theory. 3rd ed., Grad. Texts in Math. 173, Springer-Verlag, Berlin 2005. Zbl 1086.05001 MR 2159259

[11] D. Gaboriau, Coût des relations d'équivalence et des groupes. Invent. Math. 139 (2000), 41-98. Zbl 0939.28012 MR 1728876

[12] S. Gao and S. Jackson. Countable abelian group actions and hyperfinite equivalence relations. Invent. Math., to appear.

[13] E. Glasner, J.-P. Thouvenot, and B. Weiss, Every countable group has the weak Rohlin property. Bull. London Math. Soc. 38 (2006), 932-936. Zbl 1116.28013 MR 2285247

[14] M. Gromov, Topological invariants of dynamical systems and spaces of holomorphic maps. I. Math. Phys. Anal. Geom. 2 (1999), 323-415. Zbl 1160.37322 MR 1742309

[15] J. L. Gross and J. Yellen (eds.), Handbook of graph theory. CRC Press, Boca Raton, FL, 2004. Zbl 1036.05001 MR 2035186 
[16] G. Hjorth, Mixing actions of groups with the Haagerup approximation property. Fund. Math. 203 (2009), 47-56. Zbl 1168.03036 MR 2491781

[17] G. Hjorth and A. S. Kechris, Rigidity theorems for actions of product groups and countable Borel equivalence relations. Mem. Amer. Math. Soc. 177 (2005), no. 833. Zbl 1094.37002 MR 2155451

[18] A. J. Hoffman. On eigenvalues and colorings of graphs. In Graph theory and its applications, Academic Press, New York 1970, 79-91. Zbl 0221.05061 MR 0284373

[19] W. Imrich and N. Seifter, A bound for groups of linear growth. Arch. Math. (Basel) 48 (1987), 100-104. Zbl 0584.20025 MR 878419

[20] S. Jackson, A. S. Kechris, and A. Louveau, Countable Borel equivalence relations. $J$. Math. Log. 2 (2002), 1-80. Zbl 1008.03031 MR 1900547

[21] H. A. Jung and M. E. Watkins, On the connectivities of finite and infinite graphs. Monatsh. Math. 83 (1977), 121-131. Zbl 0381.05036 MR 0460163

[22] A. S. Kechris, Unitary representations and modular actions. Zap. Nauchn. Sem. S.Peterburg. Otdel. Mat. Inst. Steklov. (POMI) 326 (2005), 97-144; English transl. J. Math. Sci. 140 (2007), 398-425. Zbl 1086.22010 MR 2183218

[23] A. S. Kechris, Global aspects of ergodic group actions. Math. Surveys Monogr. 160, Amer. Math. Soc., Providence, RI, 2010. Zbl 2583950 MR 2583950

[24] A. S. Kechris, Weak containment in the space of actions of a free group. Israel J. Math. 189 (2012), 461-507. Zbl 06105481 MR 2931406

[25] A. S. Kechris and B. D. Miller, Topics in orbit equivalence. Lecture Notes in Math. 1852, Springer-Verlag, Berlin 2004. Zbl 1058.37003 MR 2095154

[26] A. S. Kechris, S. Solecki, and S. Todorcevic, Borel chromatic numbers. Adv. Math. 141 (1999), 1-44. Zbl 0918.05052 MR 1667145

[27] D. Kerr and M. Pichot, Asymptotic abelianness, weak mixing, and property T. J. Reine Angew. Math. 623 (2008), 213-235. Zbl 1155.22007 MR 2458045

[28] H. Kesten, Symmetric random walks on groups. Trans. Amer. Math. Soc. 92 (1959), 336-354. Zbl 0092.33503 MR 0109367

[29] A. Kłopotowski, M. G. Nadkarni, H. Sarbadhikari, and S. M. Srivastava, Sets with doubleton sections, good sets and ergodic theory. Fund. Math. 173 (2002), 133-158. Zbl 1004.60002 MR 1924811

[30] M. Laczkovich, Closed sets without measurable matching. Proc. Amer. Math. Soc. 103 (1988), 894-896. Zbl 0668.28002 MR 947676

[31] E. Lindenstrauss and B. Weiss, Mean topological dimension. Israel J. Math. 115 (2000), 1-24. Zbl 0978.54026 MR 1749670

[32] A. Lubotzky, R. Phillips, and P. Sarnak, Hecke operators and distributing points on the sphere. I. Comm. Pure Appl. Math. 39 (1986), Suppl., S149-S186. Zbl 0619.10052 MR 861487

[33] A. Lubotzky, R. Phillips, and P. Sarnak, Ramanujan graphs. Combinatorica 8 (1988), 261-277. Zbl 0661.05035 MR 963118

[34] A. Lubotzky and A. Zuk, On property $(\tau)$. See http://www.ma.huji.ac.il/ alexlub/. 
[35] R. Lyons and F. Nazarov, Perfect matchings as IID factors on non-amenable groups. European J. Combin. 32 (2011), 1115-1125. Zbl 1229.05115 MR 2825538

[36] G. A. Margulis, Explicit group-theoretical constructions of combinatorial schemes and their application to the construction of expanders and concentrators. Probl. Inf. Transm. 24 (1988), No.1, 39-46. Zbl 0708.05030 MR 939574

[37] J. Meier, Groups, graphs and trees. London Math. Soc. Stud. Texts 73, Cambridge University Press, Cambridge 2008. Zbl 05306923 MR 2498449

[38] A. Miller, Arnie Miller's problem list. In Set theory of the reals (Ramat Gan, 1991), Israel Math. Conf. Proc. 6, Bar-Ilan Univ., Ramat Gan 1993, 645-654. Zbl 0828.03017 MR 1234292

[39] B. D. Miller, Measurable chromatic numbers. J. Symbolic Logic 73 (2008), 1139-1157. Zbl 1157.03025 MR 2467208

[40] M. Morgenstern, Existence and explicit constructions of $q+1$ regular Ramanujan graphs for every prime power q. J. Combin. Theory Ser. B 62 (1994), 44-62. Zbl 0814.68098 MR 1290630

[41] A. Nevo, Pointwise ergodic theorems for actions of groups. In Handbook of dynamical systems, Vol. 1B, Elsevier, Amsterdam 2006, 871-982. Zbl 1130.37310 MR 2186253

[42] G. A. Niblo and M. A. Roller, Groups acting on cubes and Kazhdan's property (T). Proc. Amer. Math. Soc. 126 (1998), 693-699. Zbl 0906.20024 MR 1459140

[43] D. S. Ornstein and B. Weiss, Entropy and isomorphism theorems for actions of amenable groups. J. Analyse Math. 48 (1987), 1-141. Zbl 0637.28015 MR 910005

[44] J. Stallings, Group theory and three-dimensional manifolds. Yale Math. Monogr. 4, Yale University Press, New Haven 1971. Zbl 0241.57001 MR 0415622

[45] C. T. C. Wall, The geometry of abstract groups and their splittings. Rev. Mat. Complut. 16 (2003), 5-101. Zbl 1059.20039 MR 2031877

Received July 9, 2010; revised August 2, 2011

C. T. Conley, 584 Malott Hall, Department of Mathematics, Cornell University, Ithaca, NY 14853-4201, U.S.A.

E-mail: clintonc@math.cornell.edu

A. S. Kechris, Department of Mathematics, California Institute of Technology, Pasadena, CA 91125, U.S.A.

E-mail: kechris@caltech.edu 